\title{
2 A ciliopathy complex builds distal appendages to initiate ciliogenesis
}

3

Dhivya Kumar ${ }^{1}$, Addison Rains ${ }^{2}$, Vicente Herranz-Pérez ${ }^{3,4}$, Quanlong Lu ${ }^{11}$, Xiaoyu Shi, ${ }^{5,6}$, Danielle L. Swaney,8,10, Erica Stevenson 8,9,10, Nevan J. Krogan,8,9,10 , Bo Huang ${ }^{5,7}$, Christopher Westlake ${ }^{11}$, Jose Manuel Garcia-Verdugo ${ }^{3}$, Bradley Yoder $^{2}$ and Jeremy F. Reiter ${ }^{1,7}$

${ }^{1}$ Department of Biochemistry and Biophysics, Cardiovascular Research Institute, University of California, San Francisco, San Francisco, CA, USA

${ }^{2}$ Department of Cell, Developmental, and Integrative Biology, University of Alabama, Birmingham

${ }^{3}$ Laboratory of Comparative Neurobiology, Cavanilles Institute of Biodiversity and Evolutionary Biology, University of Valencia, CIBERNED, 46980 Valencia, Spain

${ }^{4}$ Predepartamental Unit of Medicine, Faculty of Health Sciences, Universitat Jaume I, 12071 Castelló de la Plana, Spain

${ }^{5}$ Department of Pharmaceutical Chemistry, University of California, San Francisco, San Francisco, CA 94143 USA

${ }^{6}$ Department of Developmental and Cell Biology, University of California Irvine, Irvine, CA 92697 USA

${ }^{7}$ Chan Zuckerberg Biohub, San Francisco, CA 94158, USA

${ }^{8}$ Department of Cellular and Molecular Pharmacology, University of California, San Francisco, San Francisco, CA 94158, USA

${ }^{9}$ California Institute for Quantitative Biosciences, QBI, University of California, San Francisco, San Francisco, CA 94158, USA

${ }^{10} \mathrm{~J}$. David Gladstone Institutes, San Francisco, CA 94158, USA

${ }^{11}$ Center for Cancer Research, $\mathrm{NCl}$ Frederick, Laboratory of Cellular and Developmental Signaling, Frederick, MD 21702, USA.

*Correspondence: Jeremy.Reiter@ucsf.edu

Running Title: A distal centriole complex supports ciliogenesis

eTOC summary: Kumar et al. identifies a multi-protein complex called DISCO (DIStal centriole COmplex) required to nucleate distal appendages and restrain centriole elongation, essential for the initiation of cilium assembly. Without DISCO, cells fail to ciliate and transduce Hedgehog signals, critical for mammalian development. 


\section{ABSTRACT}

Cells inherit two centrioles, the older of which is uniquely capable of generating a cilium. Using proteomics and super-resolved imaging, we identified a module which we term DISCO (DIStal centriole COmplex). DISCO components CEP90, MNR and OFD1 underlie human ciliopathies. This complex localized to both distal centrioles and centriolar satellites, proteinaceous granules

41 surrounding centrioles. Cells and mice lacking CEP90 or MNR did not generate cilia, failed to 42 assemble distal appendages, and did not transduce Hedgehog signals. Disrupting the satellite 43 pools did not affect distal appendage assembly, indicating that it is the centriolar populations of 44 MNR and CEP90 that are critical for ciliogenesis. CEP90 recruited the most proximal known distal 45 appendage component, CEP83, to root distal appendages formation, an early step in ciliogenesis. 46 In addition, MNR, but not CEP90, restricted centriolar length by recruiting OFD1. We conclude 47 that DISCO acts at the distal centriole to support ciliogenesis by restraining centriole length and 48 assembling distal appendages, defects in which cause human ciliopathies.

KEYWORDS: mother centriole, distal appendages, expansion microscopy, centriole length, 51 centriolar satellites, DISCO 


\section{INTRODUCTION}

Centrioles are ancient, microtubule-based structures with two main functions: first, they are core components of the centrosome, the primary microtubule organizing center, and, second, they are the foundations for cilia, cellular antennae specialized for signaling. While the main barrel of each centriole is comprised of nine radially arranged microtubule triplets, many proteins apart from tubulin comprise the centrioles (Winey and O'Toole, 2014; Keller et al., 2005, 2009; Jakobsen et al., 2011; Andersen et al., 2003). To date, the parts list of centrioles is incomplete.

Centriole structure can vary between species, but within a particular cell type, centriole length is nearly uniform, as are centriolar number and timing of assembly (Goehring and Hyman, 2012; Kong et al., 2020). Coordination with the cell cycle entrains this uniformity: centrioles duplicate during interphase and, at mitosis, each daughter cell inherits an older, mother centriole and a younger, daughter centriole (Nigg and Holland, 2018; Breslow and Holland, 2019).

The differences in centriolar age dictates different structures and functions. Only the mother centriole can assemble a cilium, and only it possesses subdistal and distal appendages. Through the distal appendages, the mother centriole attaches to pre-ciliary vesicles, an early step in ciliogenesis (Tanos et al., 2013; Sillibourne et al., 2013; Schmidt et al., 2012b). In contrast, subdistal appendages are dispensable for ciliogenesis (Tanos et al., 2013; Mazo et al., 2016; Chong et al., 2020). Although components of appendages have been identified (Bowler et al., 2019; Yang et al., 2018; Chong et al., 2020), how they are assembled remains enigmatic.

Related to their roles in fundamental cellular processes such as cell division and intercellular communication, centriole dysfunction causes diverse human developmental disorders including ciliopathies and microcephaly (Nigg and Holland, 2018; Reiter and Leroux, 2017). For example, mutations in CEP90 (Centrosomal protein of $90 \mathrm{kDa}$, also known as PIBF1), MNR (MOONRAKER, also known as KIAA0753 or OFIP) or OFD1 cause Joubert syndrome (JBTS), a ciliopathy characterized by brainstem and cerebellar malformations (Wheway et al., 2015; Shen et al., 2020; Hebbar et al., 2018; Stephen et al., 2017; Hammarsjö et al., 2017; Coene et al., 2009). Similarly, in many cancers, the number or structure of centrioles is dysregulated (Gönczy, 2015; Marteil et al., 2018).

CEP90 has been identified as a component of centriolar satellites, protein assemblies that orbit the centrosome (Kim et al., 2012; Kodani et al., 2015). Knockdown of CEP90 alters the localization of centriolar satellites, which has been proposed to compromise ciliogenesis (Kim and

101 Rhee, 2011; Kim et al., 2012). 
We have used a combination of label retention expansion microscopy and structured illumination microscopy (LR-ExSIM) (Shi et al., 2019) and found that, in addition to being a component of centriolar satellites, CEP90 is a component of the distal end of centrioles. Proteomic analyses revealed that CEP90 is part of an evolutionarily conserved complex, which we term DISCO (DIStal centriole COmplex) that includes MNR and OFD1. Mouse genetic studies revealed that CEP90 and MNR are key regulators of mother centriole function, essential for normal vertebrate development, Hedgehog signaling and ciliogenesis. In investigating how CEP90 and MNR function in ciliogenesis, we found that both are required for the assembly of distal appendages. Mutant analysis demonstrated that the components of this distal centriole complex are recruited to the centriole in a hierarchical manner, culminating in CEP90, which recruits the most proximal distal appendage protein, CEP83, to initiate distal appendage formation. Thus, our work identifies an evolutionarily conserved complex that functions at the distal mother centriole to build distal appendages, an early and critical step in ciliogenesis.

\section{RESULTS}

\section{CEP90 localizes to centriolar satellites and a ring at the distal ends of centrioles}

CEP90 has previously been identified as a component of centriolar satellites (Kim and Rhee, 2011). We confirmed that CEP90 localizes to centriolar satellites by immunostaining human retinal pigment epithelial (RPE1) cells with antibodies to CEP90 and PCM1, a marker of centriolar satellites (Fig. 1a). In addition, a centrosomal pool of CEP90 did not co-localize with PCM1 (Fig. 1a). To more precisely identify where centrosomal CEP90 localized, we dispersed centriolar satellites with nocodazole and found that CEP90 localized with y-tubulin at centrioles (Fig. 1b). Three-dimensional structured illumination microscopy (3D-SIM) revealed that CEP90 forms rings at centrioles (Fig. 1b).

Independent of nocodazole, we disrupted centriolar satellites in RPE1 cells by deleting PCM1, a scaffold for centriolar satellites, using CRISPR/Cas9 (Supplementary Fig 1). As described previously, centriolar satellites were not detected in PCM1-- RPE1 cells (Odabasi et al., 2019). Consistent with the loss of satellites, localization of CEP90 to puncta around the centrosome was absent in PCM1-/- RPE1 cells (Fig. 1c). As in nocodazole-treated cells, the rings

131 of CEP90 at centrioles remained in PCM1-- RPE1 cells (Fig. 1c), indicating that CEP90 localizes

132 to centrioles independently of PCM1 and centriolar satellites. To confirm antibody staining 133 specificity, we immunostained exogenously expressed eYFP-tagged CEP90 in nocodazole134 treated PCM1 $^{-/}$RPE1 cells (Fig. 1d). Localization of eYFP-CEP90 resembled endogenous 
CEP90 staining, and suggested that CEP90, unlike Centrobin and CEP164, localized to both mother and daughter centrioles with similar fluorescence intensity (Fig. 1e).

Expansion microscopy involves physically expanding samples embedded in a hydrogel (Wassie et al., 2019). To precisely map the localization of CEP90 at centrioles, we combined expansion microscopy together with multi-color label retention and SIM (LR-ExSIM) to minimize signal loss during expansion and provide $\sim 30 \mathrm{~nm}$ lateral resolution (Shi et al., 2019). The distal appendage component CEP164 imaged by LR-ExSIM was comparable to data previously obtained using STORM: CEP164 formed a discontinuous ring at the mother centriole (Shi et al., 2019, 2017). Intriguingly, LR-ExSIM resolved that the ring of CEP90 is comprised of discrete puncta with a mode of nine and separated by a mean angle of $\sim 36 \mathrm{~nm}$ (Fig. 1f-i). In agreement with 3D-SIM microscopy, the CEP90 ring at the mother centriole as observed by LR-ExSIM had a smaller diameter $(228 \pm 23 \mathrm{~nm})$ and was proximal to the distal appendages. Thus, CEP90 comprises a nine-fold ring at the distal centriole (Fig 1j).

\section{CEP90, OFD1 and MNR form a distal centriole complex}

To gain insight into the function of CEP90 at the distal centriole, we proteomically identified CEP90 interactors. More specifically, we generated RPE1 cell lines stably expressing GFP (control) or YFP-CEP90. To discriminate proteins interacting with CEP90 at centriolar satellites and those interacting with CEP90 at centrioles, we also generated PCM1-/- RPE1 cell lines stably expressing GFP or YFP-CEP90 in which centriolar satellites are disrupted (Fig. 2a). We immunoprecipitated GFP from these cell lines and detected co-immunoprecipitating proteins by LC/MS/MS. Significance analysis of interactome (SAINTexpress (Teo et al., 2014)) analysis with Bayesian false discovery rate (BFDR) $<0.05$ indicated high confidence interactors (Fig. $2 b$ and c). Some CEP90 interactors, such as PCM1, CEP131 and BBS4, were identified in wild type cells, but not in $\mathrm{PCM}^{-/-}$cells, suggesting that these interactions are dependent on centriolar satellites. Consistent with this conclusion, many of these PCM1-dependent interactors, including CEP131 and BBS4, are components of centriolar satellites (Hori and Toda, 2017).

To identify centriolar interactors of CEP90, we assessed which interactors were detected in both WT and PCM1-/- RPE1 cells, as these interactions are predicted to be independent of centriolar satellites. Notably, OFD1 and MNR, previously described components of centriolar satellites and centrosomes that interact with each other (Chevrier et al., 2016), were detected as CEP90 interactors (Fig. 2b and c). Using co-immunoprecipitation, we confirmed that CEP90 interacts with PCM1, MNR and OFD1 (Fig. 2d). Phylogenetic analysis revealed that CEP90, MNR 
and OFD1 are largely co-conserved in metazoans, but absent in ecdysozoa such as Drosophila melanogaster and Caenorhabditis elegans (Fig. 2e).

To assess whether MNR and OFD1 co-localize with CEP90, we immunostained RPE1 cells. CEP90 co-localized with MNR at centriolar satellites (Fig. 3a), and in cells treated with nocodazole to disperse centriolar satellites, at centrioles (Fig. 3b). Similarly, MNR and OFD1 colocalized at centriolar satellites (Fig. 3c), and centrioles in nocodazole treated cells (Fig. 3d). Thus, like CEP90 and OFD1, MNR is a component of both centriolar satellites and centrioles.

To gain insight into the spatial organization of distal centriole proteins with respect to distal appendages, we analyzed radially oriented centrioles with 3D-SIM (Fig. 3e). Consistent with previous observations, distal appendage components CEP164 and CEP83 were organized into respectively larger and smaller rings at the mother centriole (Yang et al., 2018; Bowler et al., 2019). Of the distal centriole proteins, MNR was organized into the smallest ring (244 $\pm 29 \mathrm{~nm}$ ) (Fig. 3e and 3f). Talpid3, OFD1 and CEP90 also were organized into rings that, intriguingly, were of different diameters at the mother and daughter centrioles. At the mother centriole, rings, from largest to smallest, were comprised of Talpid3 (345 $\pm 27 \mathrm{~nm})$, OFD1 (317 $\pm 37 \mathrm{~nm}$ ) and CEP90 $(291 \pm 35 \mathrm{~nm})$. At the daughter centriole, these rings were smaller and ordered differently from largest to smallest as OFD1 (288 $\pm 21 \mathrm{~nm})$, CEP90 $(263 \pm 21 \mathrm{~nm})$ and Talpid3 (255 $\pm 24 \mathrm{~nm})$ (Fig. $3 e$ and $3 f)$, indicating that the distal centriole is reorganized when the daughter centriole matures into a mother.

Centriole biogenesis is coordinated with the cell cycle. Procentrioles form during $S$ phase and elongate in G2. To ascertain when distal centriole proteins are recruited to centrioles, we used EdU incorporation and CENPF staining to distinguish between cells in different stages of the cell cycle (Viol et al., 2020). CEP90, OFD1 and MNR localized to mother and daughter centrioles in cells in G1 (EdU negative, CENPF negative) and in S phase (EdU positive, CENPF negative) (Fig. 3g). In G2 (EdU negative, CENPF positive), both centrosomes (marked by $\gamma$ tubulin) each possessed two puncta of CEP90, OFD1 and MNR (Fig. 3g). Therefore, CEP90, OFD1 and MNR are recruited to the distal end of elongating procentrioles in G2.

\section{CEP90 and MNR are critical for vertebrate development, ciliogenesis and Hedgehog} signaling

To assess the function of CEP90 and MNR, we generated RPE1 cell lines lacking CEP90 and MNR using CRISPR/Cas9. Immunoblot analyses confirmed loss of protein in the mutant cell lines (Supplementary Fig 1 a-d). Notably, CEP90--- and MNR-/- RPE1 cells possess centrioles but both lacked cilia (Fig. 4a and 4b). As we previously identified a role for OFD1 in cilium assembly 
(Singla et al., 2010; Hunkapiller et al., 2011), we conclude that the CEP90, MNR, OFD1 distal centriole complex is essential for ciliogenesis.

To query the function of CEP90 and MNR in vertebrates, we obtained Cep90 and Mnr mutant mice from IMPC. The Cep90 mutant mice (Pibf1 1.1 (KOMP)Vlcg) contain a deletion of 7 exons, and the Mnr mutant mice (4933427D14Rik ${ }^{\mathrm{tm} 1 \text { (KOMP)Vlcg })}$ contain a deletion of 10 exons.

Heterozygous Cep90 and Mnr mice were viable and fertile, with no obvious phenotypes. Homozygous Cep90 and Mnr mice did not survive beyond embryonic day (E) 9.5. At E9.5, Cep90-

I- and $\mathrm{Mnr}^{\text {- }}$ embryos displayed pericardial edema (Fig. 4c) and unlooped, midline hearts. One key role for primary cilia is in transducing extracellular signals including Hedgehog proteins, secreted morphogens required for vertebrate development. As lack of Smoothened, a central component of the Hedgehog signal transduction pathway, or lack of cilia also produces unlooped, midline hearts (Corbit et al., 2005; Huangfu et al., 2003), we examined the presence of cilia in Cep90-and $\mathrm{Mnr}^{\mathrm{l}}$ embryos. $\mathrm{Y}$-tubulin-positive centrioles were detected in both wild type and Cep90-embryonic nodes at E8.5, but cilia were absent from Cep90-- nodes (Fig. 4d). Consistent with a defect in ciliogenesis, Cep90-- embryos also displayed attenuated expression of the Hedgehog target gene, Gli1, indicating defects in Hedgehog signal transduction (Fig. 4e). Similarly, cilia detected by ARL13B staining were absent in neural tube sections from E9.5 Mnr ${ }^{/-}$embryos (Fig. 4f). Mouse embryonic fibroblasts (MEFs) derived from Cep90 ${ }^{-/-}$and $\mathrm{Mnr}^{\mathrm{I}^{-}}$embryos recapitulated the ciliogenesis phenotype observed in CEP90 ${ }^{-/-}$and $M N R^{-/-}$RPE1 cells (Fig. 4g, h, j, k). Furthermore, qRT-PCR analysis confirmed that induction of Hedgehog target genes, Gli1 and Ptch1, in response to the Hedgehog pathway agonist SAG was abrogated in Cep90-- and $\mathrm{Mnr}^{/-}$ MEFs (Fig. 4 i, I). Thus, CEP90 and MNR are both required for vertebrate development, ciliogenesis, and Hedgehog signaling.

\section{CEP90 ciliopathy mutations affect ciliogenesis and centriolar satellite morphology}

To identify regions in CEP90 required for cilium assembly, we tested whether CEP90 lacking either the $\mathrm{N}$-terminus, C-terminus or central domain (Kim et al., 2012) could support ciliogenesis in CEP90-- RPE1 cells (Fig. 5a). Unlike expression of the full length CEP90 (FLCEP90), CEP90 lacking the $\mathrm{N}$ terminal (CEP90 ${ }^{363-757}$ ) or the C-terminal (CEP901-363) regions failed to rescue ciliogenesis defects in CEP90-/- RPE1 cells (Fig.5b,c). A construct of CEP90 lacking residues required for interaction with PCM1 (CEP90 ${ }^{\triangle 271-363}$ ) localized to the centrosomal region, and partially rescued ciliogenesis defects in CEP90-/- RPE1 cells (Fig. 5a-c). These data support a role for both $\mathrm{N}$ and $\mathrm{C}$-terminal regions of CEP90 in ciliogenesis, further highlighting its function as a critical centriolar scaffold. 
We also examined whether disease-associated variants of CEP90 compromise its ability to support cilia assembly or centriolar satellite localization. Previously, we identified a rare variant in CEP90 (CEP90 ${ }^{\mathrm{E} 89 \mathrm{Q}}$ ) associated with microcephaly (Kodani et al., 2015). Surprisingly, this variant of CEP90 displayed normal localization to centrioles and centriolar satellites (Fig. 5c), but failed to support ciliogenesis in CEP90/- RPE1 cells (Fig. 5b,c). We also assessed two mutant forms of CEP90 (CEP90 ${ }^{\text {R405Q }}$ and CEP90 ${ }^{\mathrm{D} 637 \mathrm{~A}}$ ) implicated in Joubert syndrome (Wheway et al., 2015) (Fig.5b,c). Interestingly, both Joubert associated variants of CEP90 perturbed centriolar satellite morphology (Fig. 5c), and showed reduced ability to support ciliogenesis in CEP90-1- RPE1 (Fig. $5 b)$. Thus, our data suggest that distinct human disease-associated mutations in CEP90 differentially compromise centriolar satellite morphogenesis and ciliogenesis.

To identify regions of CEP90 required for interaction with DISCO components, OFD1 and MNR, we performed co-immunoprecipitation experiments with CEP90 truncation constructs. Both OFD1 and MNR preferentially interacted with the C-terminal region of CEP90 (CEP90 ${ }^{363-757}$ ), CEP90 lacking the C-terminal (CEP901-363) failed to immunoprecipitate OFD1 and MNR (Fig 5dg). Therefore, the C-terminal region of CEP90 (in which mutations cause Joubert syndrome) is critical for interaction with DISCO subunits, OFD1 and MNR (Wheway et al., 2015).

\section{MNR, but not CEP90, restricts centriolar length}

Since distal centriole proteins OFD1, Talpid3 and C2CD3 control centriole length, we analyzed $C E P 90^{-/-}$and $M_{N R^{-/-}}$centrioles labeled with Tubulin ${ }^{A c}$ and distal centriole protein CEP162 by 3D-SIM. Compared to control centrioles, centriole length was not altered in CEP90-/cells. However, centriole length was highly variable in $\mathrm{MNR}^{-/-}$cells, with $\sim 30 \%$ of $\mathrm{MNR}^{-/-}$cells containing hyper-elongated centrioles (some of which were over a micron long, Fig 6. a-c). The hyper-elongated centrioles in $M N R^{-/-}$cells possessed the distal centriole component CEP162 at one end, suggesting that proximal-distal polarity is maintained despite the elongation (Fig. 6 a). Serial section transmission electron microscopy (TEM) analysis confirmed the presence of elongated centrioles in MNR ${ }^{-1-}$ RPE1 cells (Fig. 6 d-e). Both mother and daughter centrioles (distinguished by localization of Ninein to the mother centriole-specific sub-distal appendage) were hyper-elongated in $M N R^{-/-}$cells (Fig. 6f). Therefore, MNR restrains centriole lengthening of both mother and daughter centrioles (Fig. 6f-g). previously observed in OFD1 mutant cells (Singla et al., 2010). Therefore, we examined the localization of OFD1 in MNR ${ }^{-1-}$ RPE1 cells. OFD1 failed to localize to the centrioles in the absence of MNR, but not CEP90 (Fig. $6 \mathrm{~h}-\mathrm{i}$ ). Overexpressed MNR localizes to microtubules (Chevrier et 
al., 2016) and microtubule-associated MNR can sequester endogenous OFD1 (Supplementary figure 2). Therefore, MNR is necessary and sufficient to recruit OFD1 (Fig. 6h-i), and our data support a model in which MNR recruits OFD1 to restrict centriole length(Feng et al., 2017; Srivastava and Panda, 2017), with CEP90 being dispensable for centriole length control and dedicated to ciliogenesis.

CEP90 and MNR are required for the removal of CP110 and CEP97 from the mother centriole at the initiation of ciliogenesis

Ciliogenesis depends on a specialized protein transport machinery called intraflagellar transport (IFT). We examined whether IFT88 localization to basal bodies depends on CEP90 or $\mathrm{MNR}$, and found that IFT88 was decreased at the mother centriole of both CEP90-/- and $\mathrm{MNR}^{-/-}$ RPE1 cells in both serum-starved (Fig. 7 a,b) and cycling cells (Supplementary Fig. 3a-b).

A critical early step in ciliogenesis is the removal from the distal mother centriole of two proteins that can inhibit ciliogenesis, CP110 and CEP97. We tested whether CEP90 or MNR function removal of CP110 and CEP97 by examining CEP90-- and $M N R^{-1-}$ RPE1 cells. We found that cells lacking either CEP90 or MNR fail to remove CP110 and CEP97 (Fig. 7c-f). Taken

\section{CEP90 and MNR are required for ciliary vesicle docking and distal appendage assembly}

As ciliary vesicle formation contributes to removing CP110 and CEP97 from the mother centriole (Westlake et al., 2011; Lu et al., 2015), we investigated whether persistence of CP110 and CEP97 in CEP90 ${ }^{-/-}$and $M_{N} R^{-/-}$cells was due to defective recruitment of preciliary vesicles.

To assess whether CEP90 and MNR affect preciliary vesicle recruitment, we examined the localization of Myosin-Va-positive preciliary vesicles (Wu et al., 2018) to centrioles in wild type, CEP90-- and MNR ${ }^{-1-}$ RPE1 cells. Consistent with the defect in distal appendage formation, cells lacking either CEP90 or MNR showed reduced Myosin-Va at the mother centriole (Fig. 7gh). To confirm the requirement for both CEP90 and MNR in preciliary vesicle recruitment, we examined wild-type and knockout cells by serial section TEM, which confirmed that centrioles in cells lacking CEP90 or MNR fail to dock to preciliary vesicles (Fig. 7i-j and Supplementary Figures 4). Thus, CEP90 and MNR are essential for ciliary vesicle recruitment to the mother centriole, a key early step of ciliogenesis (Fig. 7k).

An early step of ciliogenesis is the acquisition of appendages by the mother centriole, defining its maturation into a basal body. Sub-distal appendages mediate the anchoring of the basal body to microtubules and regulate the spatial positioning of the cilium in the cell (Mazo et 
al., 2016). In CEP90-- and MNR ${ }^{-/-}$RPE1 cells, the localization of components of sub-distal appendages, such as Ninein and CEP170, were unaffected (Supplementary Fig 5a-d), indicating that CEP90 and MNR are dispensable for subdistal appendage formation.

A function of distal appendages is the recruitment of small preciliary vesicles to the mother centriole which fuse and give rise to the ciliary membrane (Tanos et al., 2013; Schmidt et al., 2012a). CEP90-- and $M N R^{-/-}$centrioles fail to recruit distal appendage proteins CEP83, FBF1, SCLT1, ANKRD26 and CEP164, indicating that they are essential for distal appendage formation (Fig. 8c-g). FBF1 and CEP164 failed to localize to the mother centriole in cycling CEP90-- and $M_{N R^{-/}}$cells, indicating that CEP90 and MNR organize the distal appendage irrespective of whether serum starvation initiates ciliogenesis (Supplementary Fig. 3e-h). CEP90 and MNR are not critical for distal appendage protein abundance (Supplementary Fig 6e), suggesting that they are not required for distal appendage protein stability but rather are essential for distal appendage assembly at the mother centriole.

Recent studies show that daughter centriole proteins are removed from the mother centriole to enable distal appendage assembly (Wang et al., 2018; Mahjoub et al., 2010). Since CEP90 localizes to the distal daughter centriole as well as the distal mother centriole, we hypothesized that CEP90 may interact with distal daughter centriole proteins. Co-immunoprecipitation revealed that CEP90 did interact with daughter centriole components Centrobin and CEP120 (Supplementary Fig. 5f). As removing daughter centriole proteins requires distal centriole proteins, such as Talpid3, we examined the localization of Talpid3 and found that Talpid3 is recruited to the distal centriole in both wild-type and CEP90 ${ }^{-/}$and $M N R^{-/-}$RPE1 cells (Supplementary Fig. $5 \mathrm{~g}$ and h). Similarly, Centrobin and CEP120 localized to only one centriole in both wild-type and CEP90-/- and $M N R^{-/-}$RPE1 cells (Supplementary Fig. 5 i-I). Therefore, CEP90 and MNR regulate distal appendage formation through a mechanism independent of Talpid3 recruitment or daughter centriole protein removal.

\section{CEP90 functions at the mother centriole to support distal appendage formation}

Using siRNA-mediated gene knockdown, a previous study showed that CEP90 promotes centriolar satellite accumulation in the vicinity of centrosomes. We confirmed that, in CEP90-/RPE1 cells, PCM1- positive centriolar satellites failed to accumulate around centrosomes (Fig 9a and 9b). Interestingly, loss of MNR did not affect targeting of PCM1- positive centriolar satellites to the centrosome (Fig 9a and 9b), indicating that CEP90 and MNR have some distinct functions in centriolar satellite distribution. These observations raised the interesting possibility that CEP90- 
dependent targeting of centriolar satellites to the centrosomal area promotes distal appendage formation and ciliogenesis.

As $\mathrm{PCM1}^{-/-}$cells lack centriolar satellites but retain CEP90 at centrioles (Fig. 1c), these cells can help disentangle the functions of CEP90 and MNR at centriolar satellites and centrioles. In accordance with previous observations (Wang et al., 2016; Odabasi et al., 2019), RPE1 cells lacking PCM1 displayed compromised ciliogenesis (Fig 9c). In stark contrast to CEP90-/- cells, distal appendage components CEP83, SCLT1and CEP164 localized equivalently to the mother centrioles of $\mathrm{PCM}^{-/-}$and wild type RPE1 cells (Fig 9d-i), indicating that distal appendage assembly is independent of PCM1. Furthermore, serial section transmission electron microscopy confirmed the presence of distal appendages in PCM1-- RPE1 cells (Fig 9j). As the critical centriolar satellite scaffold PCM1 is not required for either CEP90 localization to centrioles or distal appendage formation, we conclude that the centriolar satellite population of CEP90 is dispensable for distal appendage assembly.

If the centriolar satellite population is dispensable, we predicted that MNR and OFD1 recruitment of CEP90 to the distal centriole would be critical for distal appendage assembly and ciliogenesis. Indeed, we found that although CEP90 protein levels were unchanged in $M N R^{-/-}$ localized to the centrioles in the absence of CEP90 (Fig. 10 a-d).

To investigate how CEP90 can be specifically required for the formation distal appendages, we investigated whether CEP90 reorganizes distal centriole proteins at the mother centriole to promote distal appendage assembly. 3D-SIM analysis revealed that Talpid3 and OFD1 diameters were smaller in mother centrioles of CEP90-/- cells compared to WT (Fig 10e and 10f). These results support a model where CEP90 functions in the transformation of a daughter to a mother centriole by reorganizing the distal centriole.

Next, we examined whether CEP90 interacts with the distal centriole base. Coimmunoprecipitation analysis revealed an interaction between CEP90 and the proximal mostcomponent of the distal centriole, CEP83 (Fig. 10g). We propose that MNR recruits CEP90 to the distal centriole, and CEP90, in turn, recruits CEP83 to initiate distal appendage assembly (Fig. 10h). Therefore, distal centriolar proteins are recruited in a hierarchical manner, with MNR recruiting OFD1 to restrict centriole length, and CEP90 dedicated to distal appendage assembly. 
Through the process of mitosis, vertebrate daughter cells each inherit two centrioles. Yet, only the older, mother centriole templates a cilium. In no small part, this unique function of the

372 mother centriole depends on its distal appendages, acquired during the previous G2/M phase. In

373 this study, we identify a multi-protein complex which we name DISCO, comprised of CEP90,

374 OFD1 and MNR and critical for distal appendage formation and ciliogenesis.

Consequent to their role in distal appendage formation, CEP90 and MNR are also required for subsequent events in ciliogenesis, including recruitment of the preciliary vesicles that give rise to the ciliary membrane, and loading of IFT88 (Sillibourne et al., 2013; Tanos et al., 2013). Unlike some other centriolar proteins, such as CEP120 (Tsai et al., 2019), CEP90 and MNR are specifically required for distal appendage formation, but dispensable for subdistal appendage formation, revealing that discrete mother centriolar complexes support distal and subdistal appendage assembly.

In mice, we found that CEP90 and MNR are essential for embryonic development, and particularly for Hedgehog signaling. Primary cilia are required for Hedgehog signal transduction in vertebrates. A central component of the Hedgehog pathway is the seven-pass transmembrane protein, Smoothened (Smo). Interestingly, Cep90, Mnr and Smo mutants arrested at similar points in embryonic development and displayed similar phenotypes, emphasizing the importance of CEP90 and MNR in ciliary Hedgehog signaling.

\section{MNR recruits OFD1 to regulate centriole length}

CEP90 and MNR colocalize with their interactor OFD1 at the distal ends of centrioles. We forms the innermost ring at the distal centriole and is essential for the recruitment of OFD1 and CEP90, whereas CEP90 is dispensable for the recruitment of the other complex members. Overexpressed MNR localizes to microtubules (Chevrier et al., 2016) bringing along OFD1. Therefore, we propose that MNR binds to the distal centriolar microtubules to recruit OFD1, culminating in CEP90 recruitment (Fig. 10).

We had previously demonstrated that OFD1 was essential to restrain centriolar length 398 (Singla et al., 2010). We have found that one of its partners, MNR, is also critical to restrict 399 centriolar elongation. However, its other partner, CEP90, does not control centriolar length. Thus, 400 within the complex comprised of MNR, OFD1 and CEP90, the subunits have distinct functions, 401 with CEP90 dedicated to founding distal appendages. Consequently, the requirement for MNR 402 and OFD1 in distal appendage assembly may be secondary to their roles in recruiting CEP90 to 403 the distal centriole. The most parsimonious model is that MNR (and perhaps OFD1) at the distal 
404

405

406

407

408

409

410

411

412

413

414

415

416

417

418

419

420

421

422

423

424

425

426

427

428

429

430

431

432

433

434

435

436

437

centriole recruits CEP90 to build distal appendages, and that MNR recruits OFD1 to restrict centriole elongation.

\section{CEP90 recruits CEP83 to initiate distal appendage assembly at the mother centriole}

The functions of centriolar satellites, although intimately associated with centrosomes, remain unclear (Odabasi et al., 2019; Prosser and Pelletier, 2020). CEP90 is a component of centriolar satellites, where it interacts with PCM1, the major scaffolding protein of centriolar satellites (Kim et al., 2012). Like CEP90, its interactors MNR and OFD1 are centriolar satellite proteins. As loss of CEP90 disrupts the peri-centrosomal localization of centriolar satellites, we initially hypothesized that centriolar satellites transport CEP90 to centrioles, or that CEP90 acts at centriolar satellites to transport distal appendage components to the mother centriole. In PCM11- RPE1 cells, centriolar satellites are disrupted but CEP90 remains at the distal centriole, indicating that satellites are dispensable for CEP90 localization at centrioles. Moreover, to our surprise, we found that PCM1/- RPE1 cells assemble distal appendages. Thus, centriolar satellites (and CEP90 at centriolar satellites) are dispensable for distal appendage assembly, indicating that CEP90 functions at the distal centriole to build distal appendages.

A combination of expansion and structured illumination super-resolution microscopy revealed that CEP90 decorates the distal centriole in a discontinuous ring-like pattern with ninefold symmetry; this ring of CEP90 is proximal to and smaller than the ring of CEP164. While antibodies to endogenous CEP90 label the daughter centriole more heavily than the mother centriole, epitope-tagged CEP90 localizes equivalently to both the mother and daughter centrioles. We speculate that reduced accessibility of the CEP90 antibody once distal appendages are assembled at the mother centriole may limit endogenous CEP90 immunofluoresence.

At the centriole, CEP90 interacts with and is critical for the recruitment of the distal appendage component CEP83. As CEP83 is the root of the distal appendage (Tanos et al., 2013), CEP90 is required to initiate distal appendage formation. In support of this conclusion, no distal appendage-like structures were observable in serial section transmission electron micrographs of cells lacking CEP90. These data raise the tantalizing possibility that the nine-fold ring of CEP90 at the distal centriole templates the assembly of the distal appendages.

Super-resolved microscopy also revealed that OFD1 and Talpid3 are structurally different in the mother and daughter centrioles, with the diameter of both rings increasing upon transition from daughter to mother. CEP90 is essential for OFD1 and Talpid3 rings to dilate to the mother centriole-specific diameter. We propose that this CEP90-dependent reorganization of distal centriole directs adoption of mother centriole-specific properties, including distal appendage 
assembly and ciliogenesis. Future work will focus on establishing how CEP90 reorganizes OFD1 and Talpid3 at the distal mother centriole to initiate distal appendage assembly.

In addition to MNR, OFD1 and CEP90, Talpid3 and C2CD3 localize to the distal centriole and are required for distal appendage formation (Singla et al., 2010; Thauvin-Robinet et al., 2014; Ye et al., 2014; Wang et al., 2018). We did not identify C2CD3 or Talpid3 in our proteomic analysis, suggesting that they might be part of a distinct, distal centriole complex (Tsai et al., 2019). Although CEP90 interacts with daughter centriole proteins CEP120 and Centrobin, Talpid3 recruitment, and the subsequent removal Centrobin and CEP120 from the distal mother centriole occurs normally in CEP90-/- RPE1 cells. Therefore, CEP90 acts independently or downstream of Talpid3 recruitment and daughter centriole protein removal to regulate distal appendage assembly.

Mutations in CEP90 cause Joubert syndrome (Kodani et al., 2015; Wheway et al., 2015; Hebbar et al., 2018), while OFD1 and MNR cause Orofaciodigital and Joubert syndrome (Singla et al., 2010; Stephen et al., 2017; Chevrier et al., 2016; Coene et al., 2009). Joubert and Orofaciodigital syndromes have partially overlapping clinical features. Ultrastructural studies have identified a cogwheel-like structure at the distal domain of human centrioles (Paintrand et al., 1992; Ibrahim et al., 2009), raising the possibility that DISCO may be components of this structure. We propose that DISCO both controls centriolar length and builds distal appendages, and that inherited defects in this cogwheel that compromise these critical functions attenuate ciliogenesis, Hedgehog signaling and embryonic patterning, resulting in Joubert and Orofaciodigital syndromes.

\section{MATERIAL AND METHODS}

\section{Mouse lines}

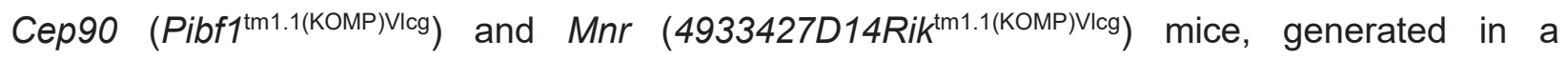
C57BL/6NJ background were obtained from the International Mouse Phenotyping Consortium. Mice were housed in a barrier facility with veterinary supervision and given food and water ad libitum. All mouse protocols were approved by the Institutional Animal Care and Use Committee at the University of California, San Francisco and the University of Alabama, Birmingham.

\section{Cell lines and cell culture}

Human retinal epithelial (RPE1-hTERT) cells were cultured in DMEM/F12 (Thermo Fisher; catalog 10565042 ) supplemented with $10 \%$ FBS at $37^{\circ} \mathrm{C}$ in $5 \% \mathrm{CO}_{2}$. To induce ciliation, cells 
were serum starved in Opti-MEM reduced serum media for indicated times. 293T cells were cultured in DMEM supplemented with $10 \%$ FBS and $1 \mathrm{X}$ GlutaMAX at $37^{\circ} \mathrm{C}$ in $5 \% \mathrm{CO}_{2}$.

472 RPE1 cell lines stably expressing eYFP-CEP90 were generated using lentiviruses containing the human CEP90 cDNA (gift from Kunsoo Rhee (Kim and Rhee, 2011; Kim et al., 2012)) in the pLVX-IRES-Puro (Clontech, Mountain View, CA) plasmid background. CEP90-1- RPE1 cells expressing $\mathrm{mNeonGreen-tagged} \mathrm{full} \mathrm{length,} \mathrm{truncation} \mathrm{and} \mathrm{disease-associated} \mathrm{mutation} \mathrm{variants}$ of CEP90 were generated using lentiviruses containing human CEP90 cDNA in a pLVX-EF1a ${ }^{\Delta_{-}}$ mNeonGreen plasmid background. Lentiviruses were generated using the Lenti-X Packaging Single Shot system (Takara Bio), according to manufacturer's guidelines. Infected cells were plated in a glass-bottom 96 well plate (Cellvis; catalog $\mathrm{P} 96-1.5 \mathrm{H}-\mathrm{N}$ ) using limiting dilution and monoclonal cell lines expressing eYFP-CEP90 were manually selected based on fluorescence.

RPE1 cells stably expressing HA-CEP83 under the control of tetracycline-inducible promoter were a gift from Barbara Tanos and Bryan Tsou (Tanos et al., 2013). CEP83 expression was induced with $1 \mu \mathrm{g} / \mathrm{ml}$ doxycycline (Fischer Chemical; Catalog BP26535) for $48 \mathrm{~h}$.

$484 \mathrm{Cep}^{-/-}$and $\mathrm{Mnr} \mathrm{r}^{--}$MEFs were derived from E8.5 embryos along with littermate WT control MEFs. Cells were cultured in DMEM supplemented with 10\% FBS (Invitrogen) and Glutamax-I (Invitrogen), and subsequently immortalized by transduction with SV40 large T antigen.

All cell lines were routinely tested for mycoplasma contamination and found negative.

\section{Generation of CEP90---, MNR ${ }^{-/-}$and PCM1-- RPE1 cells by CRISPR/Cas9 gene targeting}

RNA guided targeting of CEP90 and MNR was achieved by coexpression of Cas9 along with guide RNAs. The pSpCas9 (BB)-2A-GFP (PX458) was a gift from Feng Zhang (Addgene plasmid \#48138; http://n2t.net/addgene:48138; RRID:Addgene_48138). The gRNA sequences used for

$494 \mathrm{PCM}^{-/-}$RPE1 cells were generated by electroporating recombinant Cas9 along with sgRNAs 495 (CRISPRevolution sgRNA EZ kit, Synthego). The sgRNA targeting exon 4 of human PCM1: 5'496 GAAAAGAAUAAGAAAAAGUU-3' was used. $1.5 \mathrm{nmol}$ sgRNA was resuspended in $15 \mu \mathrm{l}$ 497 nuclease-free TE for a final concentration of $100 \mu \mathrm{M}(100 \mathrm{pmol} / \mu \mathrm{l})$. The RNP mixture containing $4981.8 \mu \mathrm{l}$ of sgRNA with $3 \mu \mathrm{l}(90 \mathrm{pmol})$ Truecut Cas9 v2 (Thermo Fisher; catalog A36498) in a total 499 volume of $5 \mu \mathrm{l}$ was incubated at room temperature for 15 minutes. The RNP mixture was 500 electroporated into hTERT-RPE1 cells using the Neon transfection system (Thermo Fisher) 
501

502

503

504

505

506

507

508

509

510

511

512

513

514

515

516

517

518

519

520

521

522

523

524

525

526

527

528

529

530

according to manufacturer's instruction using the following parameters: $1350 \mathrm{~V}$ pulse voltage, 20 ms pulse width and 2 pulses. All electroporated cells were serially diluted, and single colonies screened by western blotting, immunofluorescence and PCR analyses. For genotyping, the following PCR primers were used: 5'-TGGGATGCACTAAATTGCCTA-3' and 5'TTACCTGCCGTTTGAAGACA-3' for CEP90 alleles, 5'- TCCAGTGAACCAACTCACAGA-3' and 5'-TAGGAGCGTGGCTGTGTCTAT -3' for MNR alleles, and 5'- ACAGGCCATGTTAATTTTTGCT -3' and 5'- CCATCCCCAGTGATTAAAATTC -3' for PCM1 alleles. PCR products were cloned and sequenced.

\section{Plasmids and transfections}

Myc-DDK tagged Mnr (4933427D14Rik) cDNA in cloned in a pCMV6 plasmid was obtained from Origene (MR211309). hTERT-RPE1 cells were transfected using TransIT-LT1 transfection reagent (Mirus Bio) according to manufacturer guidelines.

For co-immunoprecipitation experiments in Fig. 5, C-terminal GFP-2x Strep-tagged full length and truncation versions of CEP90 were cloned into pLVX-EF1a-IRES-Puro backbone and transfected into 293T cells using TransIT-293 transfection reagent (Mirus Bio) according to manufacturer guidelines.

\section{Antibodies}

The list of primary antibodies and the dilution they were used at are listed in Supplementary Table 1. Secondary antibodies conjugated to Alexa Fluor 488, 568 and 647 were purchased from Thermo Fisher Scientific, and used at a 1:500 dilution.

\section{Immunofluorescence}

For immunostaining, cells were fixed with either $100 \%$ cold methanol for 3 minutes or $4 \%$ paraformaldehyde in dPBS for 15 minutes at room temperature, and then incubated in blocking buffer (2.5\% BSA, $0.1 \%$ Triton-X 100 in PBS) for 1 hour at room temperature. Paraformaldehyde fixed cells were permeabilized with $0.1 \%$ Triton X-100 in PBS for 15 minutes at room temperature prior to addition of blocking buffer. Coverslips were incubated with primary antibodies in blocking buffer overnight at $4{ }^{\circ} \mathrm{C}$, washed three times with PBS and incubated in secondary antibodies in blocking buffer for 1-2 h at room temperature. Nuclei were counterstained with Hoechst 33352 (Thermo Fisher Scientific; catalog H3570). Coverslips were washed three times with PBS and mounted with Prolong Diamond (Thermo Fisher Scientific; catalog P36961). 
531 In experiments requiring disruption of the microtubule cytoskeleton, cells were treated with $20 \mu \mathrm{M}$

532 Nocodazole (Sigma Aldrich; catalog SML1665) for $2 \mathrm{~h}$ at $37^{\circ} \mathrm{C}$ prior to fixation.

533 Identification of cell cycle stages was performed as described previously (Viol et al., 2020), using 534 the Click-iT EdU Alexa Fluor 555 imaging kit (Life Technologies). Briefly, PCM1/- RPE1 cells were 535 treated with $10 \mu \mathrm{M}$ EdU for 30 minutes and fixed in 100\% cold methanol for 3 minutes. The Click536 IT reaction was performed according to manufacturer guidelines, and samples subsequently 537 processed for indirect immunofluorescence. Cells lacking CENPF and EdU in the nucleus were 538 categorized as G1. EdU-positive, CENPF-negative cells were categorized as S, while cells with nuclear CENPF staining, without EdU staining were classified as being in G2.

\section{Super-resolution microscopy}

3D-SIM and 2D-SIM was performed using the DeltaVision OMX-SR microscope (GE Healthcare) using the 60x/1.42 NA oil immersion PSF objective and three sCMOS cameras. Immersion oil with refractive index of 1.518 was used for most experiments. Z stacks of 5-6 $\mu \mathrm{m}$ were collected using a $0.125 \mu \mathrm{m}$ step size. Raw images were reconstructed using SoftWorx 6.5.2 (GE Healthcare) using default parameters.

Label-retention expansion microscopy was performed as described previously (Shi et al., 2019). to induce ciliation for $24 \mathrm{~h}$ prior to fixation. After incubation with primary antibodies, cells were incubated with appropriate secondary antibodies conjugated to NHS-MA-biotin or NHS-MA-DIG. After gel polymerization and proteinase $\mathrm{K}$ digestion, gels were stained with fluorescently labeled streptavidin or digitonin antibodies and images were acquired on the DeltaVision OMX-SR.

\section{Fluorescence intensity measurements and statistical analyses}

554 For fluorescence intensity measurements, $z$ stacks were acquired on the DeltaVision OMX-SR using widefield settings. Identical laser power and exposure settings were used for control and experimental samples. Average intensity projections were generated using Image J (Schneider et al., 2012), and images transferred to CellProfiler image analysis software (McQuin et al., 2018). Cilia number were quantified by using Hoechst stained nuclei to count the total number of cells, and a cilia marker (ARL13B/Tubulin ${ }^{A c}$ ) to identify cilia using the object identification module in

560 CellProfiler using difference in signal intensity and size to segment cilia. For quantification of 561 centrosomal intensity, a mask around the centrosomal area was generated using a centrosomal 562 marker ( $\gamma$-tubulin/FOP) to identify the centrosome in CellProfiler. This centrosomal mask was 
used to determine fluorescence intensity (integrated intensity) and area (in pixels) for the channel of interest. Fluorescence intensity values in a pericentrosomal area were used to measure background. Cells were serum starved for $24 \mathrm{~h}$ prior to fixing, to synchronize cells in G0/G1 and eliminate cell cycle-dependent alterations in centrosomal proteins. Data were exported to Microsoft Excel, and graphs generated in GraphPad Prism 8.

Statistical analyses were performed using GraphPad Prism 8. Results represented are mean \pm standard deviation. Statistical differences between data sets were analyzed using one-way Anova with Tukey's multiple comparison tests or a two-tailed unpaired student's t test. Data distribution was assumed to be normal but this was not formally tested. $\mathrm{P}$ value $<0.05$ was considered significant and indicated with an asterisk. NS denotes not significant.

\section{Transmission electron microscopy}

For electron microscopy, cells were plated on 8-well Permanox slides (Nunc; catalog177445), gently washed in $0.1 \mathrm{M}$ phosphate buffer $(\mathrm{PB})$ and fixed in 3.5\% EM-grade glutaraldehyde (Electron Microscopy Sciences; catalog 16210) in 0.1M Phosphate buffer (PB) for 10 minutes at $37^{\circ} \mathrm{C}$. Fixative was removed, replaced with fresh fixative, and samples incubated at $4{ }^{\circ} \mathrm{C}$ for $1 \mathrm{~h}$. Slides were washed three times with 0.1 M PB and processed for TEM as described previously (Singla et al., 2010).

\section{Co-immunoprecipitation and Western Blotting}

Cells grown in $10 \mathrm{~cm}$ petri dishes were washed twice with ice-cold dPBS and lysed for 30 minutes on ice with frequent pipetting. Lysis buffer used was $10 \mathrm{mM}$ Tris/Cl pH 7.5, $150 \mathrm{mM} \mathrm{NaCl}, 0.5 \mathrm{mM}$ EDTA, $0.5 \%$ Nonidet P40 substitute supplemented with 1x protease inhibitor (Roche cOmplete mini, EDTA free; catalog 4693159001) and 1x phosphatase inhibitor (Thermo Fisher PhosStop; catalog 4906845001 ) cocktails. Lysates were cleared by centrifugation at $17,000 \mathrm{~g}$ for 10 minutes at $4{ }^{\circ} \mathrm{C}$. GFP-trap magnetic agarose (Chromotek; catalog gtma-10) was used for eYFP-CEP90 co-immunoprecipitation assays, and HA-magnetic beads (Pierce; catalog 88837) for CEP83-HA co-immunoprecipitation assays. Co-immunoprecipitation assays were performed according to manufacturer guidelines. Samples were separated on 4-15\% Criterion TGX Precast gels (BioRad), and transferred to Immobilon PVDF membranes $(0.45 \mu \mathrm{m}$ pore size $)$ for chemiluminescence detection.

\section{Mass spectrometry and data analysis}

Eluates obtained after co-immunoprecipitation were reduced by the addition of $1 \mathrm{mM} \mathrm{DTT}$ at $60^{\circ} \mathrm{C}$ for 15 minutes, cooled to room temperature, alkylated by the addition of $3 \mathrm{mM}$ iodoacetamide for 
595

596

597

598

599

600

601

602

603

604

605

606

607

608

609

610

611

612

613

614

615

616

617

618

619

620

621

622

623

624

625

626

627

45 minutes in the dark. Alkylation was quenched by the addition of $3 \mathrm{mM}$ DTT and proteins were digested overnight at $37^{\circ} \mathrm{C}$ with $1 \mu \mathrm{g}$ trypsin $(0.5 \mu \mathrm{g} / \mu \mathrm{l}$; Promega $)$. Following digestion, peptides were acidified with trifluoroacetic acid $(0.5 \%$ final, $\mathrm{pH}<2)$, desalted using UltraMicroSpin Columns (PROTO 300 C18 300Å; The NEST Group) according to manufacturer's specifications, and dried under vacuum centrifugation. Samples were resuspended in $4 \%$ formic acid, $4 \%$ acetonitrile solution, and separated by a reversed-phase gradient over a nanoflow column (360 $\mu \mathrm{m}$ O.D. $x 75 \mu \mathrm{m}$ I.D.) packed with $25 \mathrm{~cm}$ of $1.8 \mu \mathrm{m}$ Reprosil C18 particles with (Dr. Maisch). The HPLC buffers were $0.1 \%$ formic acid and $100 \%$ acetonitrile on $0.1 \%$ formic acid for buffer $A$ and $B$ respectively. The gradient was operated at $400 \mathrm{~nL} / \mathrm{min}$ from 0 to $28 \%$ buffer $B$ over $40 \mathrm{~min}$, followed by a column wash at $95 \% \mathrm{~B}$, with a total acquisition time of $50 \mathrm{~min}$. Eluting peptides were analyzed in on a Bruke timsTOF Pro mass spectrometry system equipped with a Bruker nanoElute high-pressure liquid chromatography system interfaced via a captiveSpray source. A data-dependent PASEF acquisition (Meier et al., 2018) method was used for data acquisition using the following parameters: $100-1700 \mathrm{~m} / \mathrm{z}$ range, $0.85-1.30 \mathrm{~V}$ s $/ \mathrm{cm}^{2}$ trapped ion mobility range, $1600 \mathrm{~V}$ spray voltage, intensities of 200,000 were repeated for in PASEF 0 times, intensities of 100,000 to 200,000 were repeated for in PASEF 5 times, intensities of less than 100,000 were repeated for in PASEF 10 times, 4 PASEF MS/MS scans with a total cycle time of $0.53 \mathrm{~s}$, and active exclusion for $0.4 \mathrm{~min}$. Data was search against the human proteome database (canonical sequences downloaded from Uniprot 3/21/2018) using MaxQuant (Cox and Mann, 2008; Prianichnikov et al., 2020). Peptide and protein identifications were filtered to $1 \%$ false-discovery rate at the peptide and protein level, and protein-protein interaction analysis was performed using SAINTexpress (Teo et al., 2014). The mass spectrometry data files (raw and search results) have been deposited to the ProteomeXchange Consortium (Deutsch et al., 2017; Perez-Riverol et al., 2019) (http://proteomecentral.proteomexchange.org) via the PRIDE partner repository with dataset identifier PXD022372.

\section{Online supplemental material}

Fig. S1 depicts CRISPR/Cas9-mediated mutations of CEP90, MNR and PCM1 and effect on corresponding proteins as assessed by immunoblotting. Fig. $\mathbf{S 2}$ shows that overexpressed MNR localizes to microtubules and sequesters endogenous OFD1. Fig. S3 shows that CEP90 and MNR regulate distal appendage assembly irrespective of whether the cell possesses a cilium. Fig. $\mathbf{S 4}$ includes serial-section TEMs of WT, Cep90-- and MNR ${ }^{-/-}$cells. Fig. S5 shows that CEP90 and MNR regulate distal appendage assembly independent of Talpid3 recruitment and removal of daughter centriole proteins. Table 1 lists all primary antibodies used in this study. 


\section{ACKNOWLEDGEMENTS}

629 We thank E Yu for help with animal husbandry and genotyping of mice used in this study. We 630 thank members of the Reiter and Yoder labs for helpful discussions. This work was supported by 631 NIH R01HD089918 to J.F.R and B.Y., NIH R01R01DE029454 and R01AR054396 to J.F.R., the 632 Valencian Council for Innovation, Universities, Science and Digital Society 633 (PROMETEO/2019/075) to J.M.G-V and the Spanish Ministry of Science, Innovation and 634 Universities (PCI2018-093062) to V.H-P, NIH NIGMS R01GM124334 to B.H., by the NIH Pathway 635 to Independence Award K99GM126136/R00GM126136 and the UCSF Mary Anne Koda-Kimble 636 Seed Award for Innovation to X.S. D.K. is supported a Jane Coffin Childs Postdoctoral Fellowship, 637 and the Program for Breakthrough Biomedical Research Award, which is partially funded by the 638 Sandler Foundation. B. H. and J.F.R. are Chan Zuckerberg Biohub Investigators.

639 The authors declare no competing financial interests.

640 Author contributions: Conceptualization: D. Kumar, B. Yoder and J.F Reiter; data acquisition: 641 D. Kumar, A. Rains, V. Herranz-Perez, Q. Lu, X. Shi, D.L. Swaney, E. Stevenson; funding 642 acquisition: D. Kumar, N. J. Krogan, C. Westlake, J. M. Garcia-Verdugo, B. Huang, B. Yoder and 643 J.F. Reiter; writing - original draft: D. Kumar; writing - review and editing: D. Kumar, A. Rains, V. 644 Herranz-Perez, B. Yoder, J.F. Reiter.

645 FIGURE LEGENDS

646 Figure 1. CEP90 localizes to centriolar satellites and the distal centriole. a. 3D-SIM of 647 immunostained RPE1 cells reveals localization of CEP90 (yellow) at centriolar satellites (PCM1, 648 cyan) and centrioles (Tubulin ${ }^{\mathrm{Ac}}$, magenta) in wild type, cycling RPE1 cells. Scale bar $=1 \mu \mathrm{m} . \mathbf{b}$. 649 Treatment of serum-starved RPE1 cells with nocodazole disperses the centriolar satellites, 650 highlighting 3D-SIM of CEP90 (yellow) rings at centrioles ( $\mathrm{Y}$-tubulin, magenta). Distal appendage 651 component CEP164 (cyan) indicates the mother centriole in C and D. Scale bar $=1 \mu \mathrm{m}$. c. 3D652 SIM of serum-starved PCM1-/- RPE1 cells shows that CEP90 (yellow) localizes to centrioles ( $\gamma^{-}$ 653 tubulin, magenta) independent of centriolar satellites. Scale bar of main panel and insets $=1 \mu \mathrm{m}$ 654 and $0.5 \mu \mathrm{m}$ respectively. d. 3D-SIM confirms localization of CEP90 (yellow) at centrioles ( $\gamma$ 655 tubulin, magenta) in nocodazole-treated, serum-starved eYFP-CEP90 expressing PCM1/- RPE1 656 cells. Scale bar $=1 \mu \mathrm{m}$. e. Quantification of eYFP-CEP90, Centrobin and CEP164 fluorescence 657 intensity at daughter (DC) and mother (MC) centrioles from $n=10-20$ cells. Horizontal lines 658 indicate means \pm SEM. Asterisks indicate $p<0.05$ determined using unpaired $t$ test and ns $=$ not 659 significant. f. LR-ExSIM of RPE1 cells immunostained for CEP90 (yellow) and CEP164 
660

661

662

663

664

665

666

667

668

669

670

671

672

673

674

675

676

677

678

679

680

681

682

683

684

685

686

687

688

689

690

691

692

(magenta) reveals that rings of CEP90 are comprised of discrete puncta. CEP90 rings are smaller and more proximal to CEP164 rings. Scale bar $=1 \mu \mathrm{m}$. g. Example of an LR-ExSIM image of a radially oriented centriole used to quantify the number and angle between adjacent puncta of CEP90. Scale bar $=0.5 \mu \mathrm{m}$. $\mathbf{h}$. Histogram of number of discrete puncta of CEP90 and CEP164 observed per centriole in LR-ExSIM images. $n=12-17$ measurements. i. Histogram of the angular spacing between adjoining centriolar CEP90 puncta observed by LR-ExSIM. $n=66$ measurements. j. Schematic of the ring of CEP90 punctae (yellow), distal appendages (magenta), and sub-distal appendages (blue) at the distal centriole. CEP90 decorates the distal end of mother and daughter centrioles.

Figure 2. CEP90 forms a complex with OFD1 and MNR. a. Schematic depicting workflow used to identify CEP90 interactors at centriolar satellites and centrioles using WT and PCM1-/ RPE1 cells. PCM1/-- cells localize CEP90 to the distal centriole and WT cells localize CEP90 to the distal centriole and centriolar satellites. b. Venn diagram comparing high confidence interactors of CEP90 in WT and PCM1-- RPE1 cells. c. Interactome dot representation of selected CEP90 interactors identified in the proteomic screen. Hits were grouped based on their cellular localization. Average number of peptide spectra is represented by dot shade. Abundance of a peptide spectrum produced in relation to the most abundant spectrum is depicted by dot size. Bayesian false discovery rate (BFDR) is represented by rim color. $\mathbf{d}$. Immunoblot of a subset of CEP90 interactions identified by proteomics were validated by co-immunoprecipitation (IP). CEP90 interacts with PCM1, OFD1 and MNR, but not $\alpha$-tubulin or GAPDH. FT, flow through. Specific MNR band is indicated with an asterisk. The top band is nonspecific as it is undiminished in the MNR knockout cell lysates. e. Coulson plot showing the phylogenetic distribution of a subset of centriolar proteins in select ciliated metazoan species. Orthologs identified with high confidence are indicated with a filled circle, and a subset of CEP90 interactors further explored in this study are highlighted in blue. The dendrogram on top (made using iTOL (Ciccarelli et al., 2006)) shows the evolutionary relationship between species.

Figure 3. CEP90 co-localizes with OFD1 and MNR. a. Immunostaining of RPE1 cells for CEP90 (yellow), MNR (cyan) and y-tubulin (magenta) demonstrating that CEP90 co-localizes with MNR at centriolar satellites. Scale bar $=2 \mu \mathrm{m}$. b. 3D-SIM of RPE1 cells treated with nocodazole to disperse centriolar satellites highlights co-localization of CEP90 (yellow) and MNR (cyan) at centrioles ( $\gamma$-tubulin, magenta). Scale bar $=1 \mu \mathrm{m}$. c. Immunostaining of OFD1 (yellow), MNR (cyan) and y-tubulin (magenta) reveals CEP90 and MNR co-localization at centriolar satellites. Scale bar $=2 \mu \mathrm{m}$. d. 3D-SIM of immunostained cells treated with nocodazole reveals a ring of 


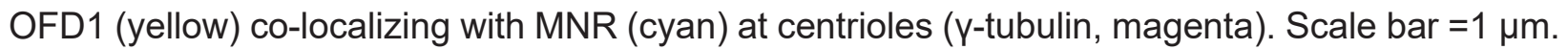
e. Measurements of ring diameters measured in 3D-SIM images. For distal centriole proteins, ring diameters were measured at the mother (MC) and daughter centriole (DC). Scatter dot plot shows mean \pm SD. Asterisks indicate $p<0.05$, and $n s$ indicate not significant determined using unpaired $t$ test. $n=13-22$ centrioles. $f$. Schematic representation of a radial view of a mother centriole with distal appendages (purple), OFD1 (orange), CEP90 (yellow) and MNR (green). g. Localization of distal centriole proteins (yellow) to centrioles ( $\gamma$-tubulin, magenta) is cell cycle dependent. Cells in $\mathrm{G} 1$ were identified as lacking both EdU and CENPF staining, cells in $\mathrm{S}$ as being positive for EdU but lacking CENPF, and cells in G2 as being positive for CENPF but lacking EdU staining. Two puncta of CEP90, OFD1 and MNR localized were observed at one centrosome during G1 and $\mathrm{S}$, and four puncta at two centrosomes after centrosome duplication during G2. Scale bar of main panel and insets $=5 \mu \mathrm{m}$ and $0.5 \mu \mathrm{m}$ respectively.

Figure 4. CEP90 and MNR are essential for ciliogenesis. a. WT, CEP90-- and $M N R^{-/-}$serum starved RPE1 cells were immunostained for cilia (ARL13B, magenta), centrosomes ( $\gamma$-tubulin, yellow) and nuclei (Hoechst, blue). Scale bar $=10 \mu \mathrm{m}$. b. Quantification of ciliation frequency of WT, CEP90-/- and MNR ${ }^{-/-}$RPE1 cells serum starved for times indicated. $\mathrm{n}>100$ cells from two biological replicates. c. Images of control, Cep90/-- and $\mathrm{Mnr}^{/-}$embryos at embryonic day (E) 9.5. Arrows point to pericardial edema observed in mutant embryos. d. Whole-mount immunostaining of nodes of littermate control and Cep90-- embryos at E8.5 for centrosomes ( $\mathrm{\gamma}$-tubulin, yellow),

712 cilia (Tubulin ${ }^{\mathrm{Ac}}$, magenta) and nuclei (Hoechst, blue). Arrows in control image point to cilia 713 projecting into the node lumen, which are absent in Cep90-/- embryos. Scale bar of main panel 714 and insets $=5 \mu \mathrm{m}$ and $2 \mu \mathrm{m}$ respectively. e. In situ hybridization for Gli1 in E8.5 littermate control 715 and Cep90-- embryos, revealing decreased expression in the absence of CEP90 indicative of 716 disrupted Hedgehog signaling. Scale bar = $5 \mu$ m. f. Immunostaining of E9.5 neural tube sections 717 of littermate control and $\mathrm{Mnr}^{\mathrm{l}}$ embryos for centrosomes (FOP, yellow), cilia (ARL13B, magenta) 718 and nuclei (Hoechst, blue), indicating that MNR is required for ciliogenesis in vivo. Scale bar $=5$

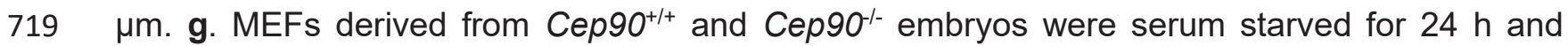
720 immunostained for cilia (ARL13B, magenta), centrosomes ( $\gamma$-tubulin, yellow) and nuclei (Hoechst, 721 blue). Scale bar $=10 \mu \mathrm{m}$. h. Quantification of ciliation frequency shows loss of cilia in Cep90-/722 MEFs. Bar graph shows mean \pm SEM. Asterisks indicate $p<0.05$ determined using unpaired $t$ 723 test. $n>100$ cells from two biological replicates. i. qRT-PCR of HH target genes Gli1 and Ptch1 724 in serum-starved Cep90 ${ }^{+/+}$and Cep90-/- MEFs stimulated with 200nM SAG for 24h relative to DMSO-treated controls. Bar graph shows mean \pm SEM. Asterisks indicate $p<0.05$ determined using unpaired t test. $\mathrm{n}=3$ biological replicates. j. MEFs derived from $\mathrm{Mnr}^{+/+}$and $\mathrm{Mnr}^{/-}$embryos 
were serum starved for $24 \mathrm{~h}$ and immunostained for cilia (ARL13B, magenta), centrosomes ( $\mathrm{Y}$ tubulin, yellow) and nuclei (Hoechst, blue). Scale bar $=10 \mu \mathrm{m}$. k. Quantification of ciliation frequency shows loss of cilia in Mnr- MEFs. Bar graph shows mean \pm SEM. Asterisks indicate $p$ $<0.05$ determined using unpaired t test. $n>100$ cells from two biological replicates. I. qRT-PCR of $\mathrm{HH}$ target genes Gli1 and Ptch1 in serum-starved $\mathrm{Mnr}^{+/+}$and $\mathrm{Mnr}^{/-}$MEFs stimulated with 200nM SAG for $24 \mathrm{~h}$ relative to DMSO-treated controls. Bar graph shows mean $\pm \mathrm{SEM}$. Asterisks indicate $p<0.05$ determined using unpaired t test. $n=3$ biological replicates.

\section{Figure 5. CEP90 ciliopathy mutations affect ciliogenesis and centriolar satellite} morphology. a. Schematic representation of full length (FL) human CEP90, truncation constructs and disease-associated mutations. Coiled-coil (CC) domains identified by MARCOIL (Zimmermann et al., 2018) at 90\% threshold are indicated in cyan. b. Quantification of ciliation frequency of CEP90-- RPE1 (control), and CEP90-- cells expressing mNeonGreen (mNG) tagged disease-variants and truncations of CEP90. Graph shows mean \pm SEM, and asterisks indicate $p$ $<0.05$ determined by one-way ANOVA. $\mathrm{n}>100$ cells from two biological replicates. C. CEP90-/RPE1 cells, and CEP90-- cells expressing the denoted mNG-tagged versions of CEP90 were serum starved for $24 \mathrm{~h}$ and immunostained for cilia (ARL13B), centriolar satellites (CEP131) and $\mathrm{mNeonGreen}$. Scale bar $=2 \mu \mathrm{m}$. d. Immunoprecipitation of GFP-tagged FL and truncation constructs of CEP90 blotted for GFP and OFD1. e. Quantification of OFD1 band intensities relative to corresponding GFP input band intensities. f. Immunoprecipitation of GFP-tagged FL and truncation constructs of CEP90 blotted for GFP and MNR. g. Quantification of MNR band intensities relative to corresponding GFP input band intensities. Asterisk represents MNR band, top band is non-specific.

Figure 6. MNR, but not CEP90, restricts centriole length. a. 3D-SIM images of WT, CEP90-/and $M_{N}{ }^{-/-}$RPE1 cells immunostained for cilia/centrioles (TubulinAc, magenta) and CEP162 (yellow), a distal centriolar protein. Scale bar of main panel and insets $=1 \mu \mathrm{m}$ and $0.5 \mu \mathrm{m}$ respectively.b. Graph of centriolar lengths measured using 3D-SIM images. Centriole lengths are longer and have a wider distribution in $\mathrm{MNR}^{-/-}$cells than in WT or CEP90-/- cells. Lines indicate mean \pm SEM. Asterisks indicate $p<0.05$ determined using one-way ANOVA. $n=14-30$ centrioles. c. Histogram of centriole lengths observed in WT, $M N R^{-/-}$and $C E P 90^{-/-}$cells. $\mathrm{n}=14-30$ centrioles. d. Serial section TEM confirms the presence of elongated centrioles in $M N R^{-/-}$RPE1 cells. Scale bar $=500 \mathrm{~nm}$. e. Centriole lengths of WT and $M N R^{-/-}$RPE1 cells measured using TEM images. Horizontal lines indicate means \pm SEM, and asterisks indicate $p<0.05$ determined using unpaired $\mathrm{t}$ test. $\mathrm{n}=8$ centrioles per condition. f. WT and $M N R^{-1-}$ RPE1 cells were serum-starved and 
immunostained with antibodies to $\mathrm{Tubulin}^{\mathrm{Ac}}$ and sub-distal appendage component Ninein to distinguish mother (MC) and daughter centrioles (DC). Graph of centriolar lengths measured using 3D-SIM. MNR restrains centriole lengthening of both mother and daughter centrioles. Lines indicate mean \pm SEM. Asterisks indicate $p<0.05$ determined using unpaired t-test. $n=10-35$ measurements. g. Schematic depicting distinct roles of CEP90 and MNR in regulating centriole length. h. 3D-SIM imaging of serum-starved WT, CEP90-/- and MNR ${ }^{-/-}$RPE1 cells immunostained for OFD1 (yellow), centrioles ( $\mathrm{y}$-tubulin, cyan) and cilia (Tubulin ${ }^{\mathrm{Ac}}$, magenta). Boxed regions are depicted in insets throughout. OFD1 localizes to centrioles in WT and CEP90 $0^{--}$, but not $\mathrm{MNR}^{-/-}$ cells. Scale bar $=1 \mu \mathrm{m}$. i. Quantification of OFD1 fluorescence intensity at centrioles in WT, $\mathrm{CEP}^{-/-}$and $\mathrm{MNR}^{-/-}$cells. Horizontal lines indicate means \pm SEM. Asterisks indicate $\mathrm{p}<0.05$ determined using one-way ANOVA. $n=64-187$ cells.

Figure 7. CEP90 and MNR are required for early ciliogenesis. a WT, CEP90-/- and $M N R^{-/-}$ RPE1 cells immunostained for IFT88 (yellow), centrioles ( $\gamma$-tubulin, cyan) and cilia (Tubulin ${ }^{A c}$, magenta. IFT88 is not recruited to the centrosome of CEP90-1- or MNR ${ }^{-1-}$ cells. b. Quantification of IFT88 fluorescence intensity at WT, CEP90-/- and $M N R^{-/-}$centrosomes. Lines indicate mean \pm SEM. Asterisks indicate $\mathrm{p}<0.05$ determined using unpaired $\mathrm{t}$ test. $\mathrm{n}=38-63$ measurements. c. WT, CEP90-/- and $M N R^{-/-}$serum-starved RPE1 cells immunostained for CP110 (yellow), centrioles ( $\mathrm{\gamma}$-tubulin, cyan) and cilia (Tubulin ${ }^{A c}$, magenta). Scale bar $=1 \mu \mathrm{m}$. d. Quantification of whether CP110 localizes to one or two centrioles. In the absence of CEP90 or MNR, CP110 continues to localize to the distal mother centriole. $\mathrm{n}>50$ cells from 2 independent experiments. Scale bar $=1$ um. e. WT, CEP90 ${ }^{-/-}$and $M N R^{-/-}$serum-starved RPE1 cells immunostained for CEP97 (yellow), Y-tubulin (cyan) and Tubulin ${ }^{A c}$ (magenta). Scale bar $=1 \mu \mathrm{m}$. f. Quantification of whether CEP97 localizes to one or two centrioles. As with CP110, CEP90 and MNR are required to remove CEP97 from the distal mother centriole. $\mathrm{n}>50$ cells from 2 independent experiments. Scale bar $=1 \mu \mathrm{m}$. g. 3D-SIM images of RPE1 cells immunostained for Myo-Va (yellow) and centrioles ( $\gamma$-tubulin, magenta). Myo-Va can not localize near centrosomes (left), can localize to preciliary vesicles (denoted centrosomal Myo-Va, middle), or can localize to the ciliary pocket (denoted ciliary MyoVa, right). Scale bar $=1 \mu \mathrm{m}$. h. Quantification of three distinct Myo-Va staining patterns in WT, CEP90 $0^{-/-}$and $M_{N R^{-/}}$cells. $\mathrm{n}>50$ cells from 2 independent experiments. i. Serial section TEM images of serum-starved Cep90 $0^{+/+}$and Cep90-- MEFs confirms the absence of preciliary vesicle docking at the Cep90--- mother centriole. Scale bar $=200 \mathrm{~nm} . \mathrm{n}=9$ cells for both genotypes. $\mathbf{j}$. Serial-section TEM images of WT and $M N R^{-/-}$RPE1 cells confirms the absence of preciliary vesicle vesicle docking at the $M N R^{--}$mother centriole. Scale bar $=250 \mathrm{~nm} . \mathrm{n}=10$ cells for WT 
and $n=6$ for $M N R^{-/-}$cells. $\mathbf{k}$. Model based on our data highlighting the role of CEP90 and MNR in maturation of the mother centriole.

Figure 8. CEP90 and MNR recruit distal appendage components to the mother centriole. 3D-SIM images and quantification of centrosomal intensity of WT, CEP90 ${ }^{-/-}$and $M N R^{-/-}$RPE1 cells immunostained for $\mathrm{Y}$-tubulin (cyan), Tubulin ${ }^{A c}$ (magenta) and distal centriole components (yellow) CEP83 (a,b), FBF1 (c,d), SCLT1 (e,f), ANKRD26 (g,h) and CEP164 (i,j). Scale bar = $1 \mu \mathrm{m}$. Horizontal lines in scatter dot plots indicate means \pm SEM. Asterisks indicate $p<0.001$ determined by one-way ANOVA. $\mathrm{n} \geq 40$ measurements per condition.

Figure 9. Centriolar satellites are dispensable for distal appendage assembly at the mother centriole. a. 3D-SIM images of WT, CEP90-- and MNR ${ }^{-/-}$RPE1 cells immunostained with antibodies to PCM1, a centriolar satellite marker, ARL13B and y-tubulin. Scale bar = $2 \mu \mathrm{m}$. b. Quantification of PCM1 intensity in the pericentrosomal area. PCM1-positive centriolar satellites

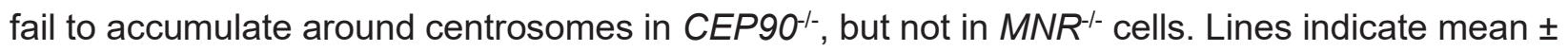
SEM. Asterisks indicate $\mathrm{p}<0.05$ determined using ordinary one-way ANOVA. $\mathrm{n}=37-44$ measurements. c. Ciliogenesis is disrupted in $P C M 1^{-/-}$RPE1 cells at $24 \mathrm{~h}$ post serum starvation. $\mathrm{n}>100$ cells from two biological replicates. d. WT and PCM1-- RPE1 cells were serum starved for $24 \mathrm{~h}$ and stained with antibodies to CEP83, $\mathrm{y}$-tubulin (centrosome marker) and Tubulin ${ }^{\mathrm{Ac}}$ (cilia marker). 3D-SIM imaging reveals ring of CEP83 at the mother centrioles in PCM1-- RPE1 cells. Scale bar $=1 \mu \mathrm{m}$. e. Quantification of CEP83 fluorescence intensity at centrioles. Scatter dot plots show mean \pm SEM. NS $=$ not significant, determined using unpaired $t$ test. $n=53-124$ measurements. f. WT and PCM1-- RPE1 cells were serum starved for $24 \mathrm{~h}$ and stained with antibodies to SCLT1, y-tubulin (centrosome marker) and ARL13B (cilia marker). 3D-SIM imaging reveals ring of SCLT1 at the mother centrioles in WT and PCM1-- RPE1 cells. Scale bar $=1 \mu \mathrm{m}$. g. Quantification of SCLT1 fluorescence intensity at centrioles. Scatter dot plots show mean \pm SEM. NS = not significant, determined using unpaired $\mathrm{t}$ test. $\mathrm{n}=26-28$ measurements. $\mathbf{h}$. WT and PCM1-- RPE1 cells were serum starved for $24 \mathrm{~h}$ and stained with antibodies to CEP164 and $\mathrm{Y}^{-}$ tubulin (centrosome marker). 3D-SIM imaging reveals ring of CEP164 at the mother centrioles in PCM1-- RPE1 cells. Scale bar $=1 \mu \mathrm{m}$. i. Quantification of CEP164 fluorescence intensity at centrioles. Scatter dot plots show mean \pm SEM. Asterisks indicate $p<0.001$, determined using unpaired t test. $n=40-60$ measurements. j. Representative TEM images of WT and PCM1-- RPE1 cells serum starved for 1 hour. Distal appendages are marked with red arrows. 
Figure 10. MNR recruits CEP90 which recruits CEP83 to build distal appendages. a. 3D-SIM imaging of WT, CEP90-- and $M N R^{-/-}$serum-starved RPE1 cells immunostained for CEP90 (yellow), $\mathrm{y}$-tubulin (cyan) and Tubulin ${ }^{A c}$ (magenta). Scale bar $=1 \mu \mathrm{m}$. b. Quantification of CEP90 fluorescence intensity at centrioles. Horizontal lines in scatter dot plots indicate means \pm SEM. Asterisks indicate $\mathrm{p}<0.0001, \mathrm{~ns}=$ not significant, determined using one-way ANOVA. $\mathrm{n}=31-37$ measurements. CEP90 fails to localize to $M N R^{-1-}$ and CEP90 ${ }^{-/-}$centrioles, although protein levels of CEP90 remained unchanged in $M N R^{-/-}$RPE1 cells (Supplementary Fig. 1d). c. 3D-SIM imaging of WT, CEP90 $0^{-/-}$and $M_{N} R^{-/-}$serum-starved RPE1 cells immunostained for MNR (yellow), y-tubulin (cyan) and CEP164 (magenta). Scale bar $=1 \mu \mathrm{m}$. d. Quantification of MNR fluorescence intensity at centrioles. Horizontal lines in scatter dot plots indicate means \pm SEM. Asterisks indicate $p<$ 0.0001, determined using one-way ANOVA. $n=73-122$ measurements. MNR localization is reduced but present at CEP90-- centrioles. e. Quantification of Talpid3 diameter at mother (MC) and daughter centrioles (DC) in serum-starved WT and CEP90--- cells. Horizontal lines in scatter dot plots indicate means \pm SEM. Asterisks indicate $p<0.05$ determined using one-way ANOVA, $\mathrm{ns}=$ not significant. $\mathrm{n}=11-16$ measurements. The Talpid3 ring diameter is increased at WT mother centrioles, but not at CEP90-- mother centrioles. f. Quantification of OFD1 diameter at mother (MC) and daughter centrioles (DC) in serum-starved WT and CEP90-- cells. Horizontal lines in scatter dot plots indicate means \pm SEM. Asterisks indicate $p<0.05$, determined using one-way ANOVA. $n=17-20$ measurements. In the absence of CEP90, the mother centriole ring of OFD1 does not expand as in wild-type cells. g. WT or RPE1 cells expressing CEP83-HA were lysed, immunoprecipitated with anti-HA antibody bound beads and immunoblotted with antibodies to HA and CEP90. $\mathbf{h}$. Model of the hierarchical recruitment of the DISCO complex. MNR recruitment of OFD1 restrains centriole elongation and MNR recruits CEP90 which, in turn, recruits CEP83, the base of the distal appendage.

\section{SUPPLEMENTARY FIGURES:}

SUPPLEMENTARY FIGURE 1. Generation of CEP90 ${ }^{-1-}, \mathrm{MNR}^{-1-}$ and $P C M 1^{-1-}$ cell lines. Sequence analysis of genomic DNA isolated from control and CEP90 ${ }^{-/-}(\mathbf{a}), \mathrm{MNR}^{- \text {- }^{-}}$(b) and $P C M 1^{-}$ I- (c) RPE1 cell lines generated using CRISPR-Cas9. Insertions and deletions, and translation products resulting from genome editing are indicated. $\mathbf{d}$. Immunoblot analysis of whole cell lysates from control, CEP90 ${ }^{-/-}, \mathrm{MNR}^{-/-}$and $P C M 1^{-/-}$RPE1 cell lines confirms loss of protein in mutant cell lines. Specific MNR band is indicated with an asterisk, top band is non-specific. 

immunostained with antibodies to myc-tag and $\alpha$-tubulin. Scale bar $=10 \mu \mathrm{m}$. b. RPE1 cells transiently transfected with myc-tagged MNR, and immunostained with antibodies to myc-tag and OFD1. Scale bar $=10 \mu \mathrm{m}$. c. RPE1 cells transiently transfected with myc-tagged MNR, and immunostained with antibodies to myc-tag and CEP90. Scale bar $=10 \mu \mathrm{m}$. d. RPE1 cells transiently transfected with myc-tagged MNR, and immunostained with antibodies to myc-tag and FOPNL. Scale bar $=10 \mu \mathrm{m}$.

SUPPLEMENTARY FIGURE 3. CEP90 and MNR regulate distal appendage assembly irrespective of ciliation status. a. Cycling WT, CEP90 $0^{-/}$and $M N R^{-1-}$ RPE1 cells stained with antibodies to IFT88, y-tubulin (centrosome marker) and Tubulin ${ }^{A c}$ (cilia and centriole marker). 3DSIM imaging reveals IFT88 at the centrosome in WT cells, but not in CEP90--- and $\mathrm{MNR}^{-1-}$ cells. Scale bar for main panel and insets $=1 \mu \mathrm{m}$. b. Quantification of IFT88 fluorescence intensity at centrioles. Scatter dot plots show mean \pm SEM. Asterisks indicate $p<0.001$, determined using one-way ANOVA. $\mathrm{n}=73-96$ measurements. c. Cycling WT, CEP90-- and MNR ${ }^{-/-}$RPE1 cells

872 immunostained for CP110 (yellow), centrioles (FOP, cyan) and Tubulin ${ }^{A c}$ (cilia and centriole marker, magenta). Scale bar $=1 \mu \mathrm{m}$. d. Quantification of CP110 foci. e. Cycling WT, CEP90-- and $M N R^{-1-}$ RPE1 cells stained with antibodies to FBF1, y-tubulin (centrosome marker) and Tubulin Ac (cilia and centriole marker). 3D-SIM imaging reveals FBF1 at the mother centriole in WT cells, but not in CEP90--- and $\mathrm{MNR}^{-/-}$cells. Scale bar for main panel and insets $=1 \mu \mathrm{m}$. $\mathrm{f}$. Quantification of FBF1 fluorescence intensity at centrioles. Scatter dot plots show mean \pm SEM. Asterisks indicate $p<0.001$, determined using one-way ANOVA. $n=21-45$ measurements. g. Cycling WT, CEP90-/and $M N R^{-/-}$RPE1 cells stained with antibodies to CEP164, y-tubulin (centrosome marker) and

880 Tubulin $^{\mathrm{Ac}}$ (cilia and centriole marker). 3D-SIM imaging reveals CEP164 at one of the two centrioles in WT cells, but not in CEP90-/- and MNR ${ }^{-1-}$ cells. Scale bar for main panel and insets = $1 \mu \mathrm{m}$. h. Quantification of CEP164 fluorescence intensity at centrioles. Scatter dot plots show mean \pm SEM. Asterisks indicate $p<0.001$, determined using one-way ANOVA. $n=37-53$ measurements. cells lacking CEP90 or MNR. a. TEM images ofCep90 $0^{+/+}$and Cep90 $0^{-/-}$MEF cells confirms the absence of preciliary vesicle vesicle docking, and distal appendages at the Cep90-1- mother centriole. Scale bar $=500 \mathrm{~nm}$. Blue arrowheads indicate subdistal appendages, and pink arrowheads indicate distal appendages. CP indicate ciliary pocket. b. TEM images of WT and 
$M N R^{-/-}$RPE1 cells confirms the absence of preciliary vesicle docking and distal appendages at the $M N R^{-1-}$ mother centriole. Centrioles are hyper-elongated in the absence of MNR. Scale bar = $500 \mathrm{~nm}$. Blue arrowheads indicate subdistal appendages, and pink arrowheads indicate distal appendages. CP indicate ciliary pocket.

SUPPLEMENTARY FIGURE 5. CEP90 regulates distal appendage assembly independent of Talpid3 recruitment and removal of Centrobin. a. 3D-SIM imaging of serum-starved WT, CEP90-- and MNR-/- RPE1 cells immunostained for Ninein (yellow), centrioles ( $\mathrm{\gamma}$-tubulin, cyan) and cilia (ARL13B, magenta). Boxed regions are depicted in insets throughout. Ninein localizes to centrioles in WT, CEP90 ${ }^{-/-}$and $M N R^{-/-}$cells. Scale bar $=1 \mu \mathrm{m}$. b. Quantification of Ninein fluorescence intensity at centrioles in WT, CEP90 ${ }^{-/-}$and $\mathrm{MNR}^{-/-}$cells. Horizontal lines indicate means \pm SEM. Asterisks indicate $p<0.05$ determined using one-way ANOVA, ns indicates not significant. $\mathrm{n}=36-45$ measurements. c. 3D-SIM imaging of serum-starved WT, CEP90-1- and $M_{N R^{-/}}$RPE1 cells immunostained for CEP170 (yellow), centrioles ( $\gamma$-tubulin, cyan) and cilia (Tubulin ${ }^{A c}$, magenta). Boxed regions are depicted in insets throughout. CEP170 localizes to centrioles in WT, CEP90 ${ }^{-/-}$and $M N R^{-/-}$cells. Scale bar $=1 \mu \mathrm{m}$. d. Quantification of CEP170 fluorescence intensity at centrioles in WT, CEP90 ${ }^{-1-}$ and $\mathrm{MNR}^{-/-}$cells. Horizontal lines indicate means \pm SEM. Asterisks indicate $\mathrm{p}<0.05$ determined using one-way ANOVA. $\mathrm{n}=73-99$ measurements. e. Immunoblot of distal appendage proteins CEP83 and CEP164 in WT, CEP90I-, MNR ${ }^{-/-}$and PCM1/- RPE1 cells. f. Co-immunoprecipitation of daughter centriole proteins Centrobin and CEP120 with CEP90. IP indicates eluate and FT indicates flow through g. WT, CEP90 ${ }^{-1-}$ and $M_{N R^{-1-}}$ RPE1 cells were serum starved for $24 \mathrm{~h}$ and stained with antibodies to Talpid3, y-tubulin (centrosome marker) and ARL13B (cilia marker). 3D-SIM imaging reveals ring of Talpid3 at mother and daughter centrioles. Scale bar $=1 \mu \mathrm{m}$ for main panels and insets. $\mathbf{h}$. Quantification of Talpid3 fluorescence intensity at centrioles. Scatter dot plots show mean \pm SEM. Asterisks indicate $p<0.001$, determined using one-way ANOVA. $\mathrm{n}=33-35$ measurements. i. WT, $\mathrm{CEP}^{-/-}$and $\mathrm{MNR}^{-/-}$serum-starved RPE1 cells immunostained for Centrobin (yellow), centrioles (FOP, cyan) and distal appendages (CEP164, magenta). Scale bar $=1 \mu \mathrm{m}$. j. Quantification of whether Centrobin localizes to one or two centrioles. $n>50$ cells from two biological replicates. CEP90 and MNR are not required to remove Centrobin from the distal mother centriole. k. WT, CEP90-/- and $\mathrm{MNR}^{-/-}$serum-starved RPE1 cells immunostained for CEP120 (yellow), centrioles (FOP, cyan) and distal appendages (CEP164, magenta). Scale bar $=1 \mu \mathrm{m}$. j. Quantification of whether CEP120 localizes to one or two centrioles. $n>50$ cells from two biological replicates. CEP90 and MNR are not required to remove Centrobin or CEP120 from the distal mother centriole. 


\section{REFERENCES}

Andersen, J.S., C.J. Wilkinson, T. Mayor, P. Mortensen, E.A. Nigg, and M. Mann. 2003. Proteomic characterization of the human centrosome by protein correlation profiling. Nature. 426:570_ 574. doi:10.1038/nature02166.

Bowler, M., D. Kong, S. Sun, R. Nanjundappa, L. Evans, V. Farmer, A. Holland, M.R. Mahjoub, H. Sui, and J. Loncarek. 2019. High-resolution characterization of centriole distal appendage morphology and dynamics by correlative STORM and electron microscopy. Nat. Commun. 10. doi:10.1038/s41467-018-08216-4.

Breslow, D.K., and A.J. Holland. 2019. Mechanism and Regulation of Centriole and Cilium Biogenesis. Annu. Rev. Biochem. 88:691-724. doi:10.1146/annurev-biochem-013118111153.

Chevrier, V., A.-L. Bruel, T.J.P. Van Dam, B. Franco, M. Lo Scalzo, F. Lembo, S. Audebert, E. Baudelet, D. Isnardon, A. Bole, J.-P. Borg, P. Kuentz, J. Thevenon, L. Burglen, L. Faivre, J.B. Rivière, M.A. Huynen, D. Birnbaum, O. Rosnet, and C. Thauvin-Robinet. 2016. OFIP/KIAA0753 forms a complex with OFD1 and FOR20 at pericentriolar satellites and centrosomes and is mutated in one individual with oral-facial-digital syndrome. Hum. Mol. Genet. 25:497-513. doi:10.1093/hmg/ddv488.

Chong, W.M., W.-J. Wang, C.-H. Lo, T.-Y. Chiu, T.-J. Chang, Y.-P. Liu, B. Tanos, G. Mazo, M.F.B. Tsou, W.-N. Jane, T.T. Yang, and J.-C. Liao. 2020. Super-resolution microscopy reveals coupling between mammalian centriole subdistal appendages and distal appendages. Elife. 9. doi:10.7554/elife.53580.

Ciccarelli, F.D., T. Doerks, C. Von Mering, C.J. Creevey, B. Snel, and P. Bork. 2006. Toward automatic reconstruction of a highly resolved tree of life. Science (80-. ). 311:1283-1287. doi:10.1126/science.1123061.

Coene, K.L.M., R. Roepman, D. Doherty, B. Afroze, H.Y. Kroes, S.J.F. Letteboer, L.H. Ngu, B. Budny, E. van Wijk, N.T. Gorden, M. Azhimi, C. Thauvin-Robinet, J.A. Veltman, M. Boink, T. Kleefstra, F.P.M. Cremers, H. van Bokhoven, and A.P.M. de Brouwer. 2009. OFD1 Is Mutated in X-Linked Joubert Syndrome and Interacts with LCA5-Encoded Lebercilin. Am. J. Hum. Genet. 85:465-481. doi:10.1016/j.ajhg.2009.09.002. 
954

955

956

957

958

959

960

961

962

963

964

965

966

967

968

969

970

971

972

973

974

975

976

977

978

979

980

981

982

983

Corbit, K.C., P. Aanstad, V. Singla, A.R. Norman, D.Y.R. Stainier, and J.F. Reiter. 2005. Vertebrate Smoothened functions at the primary cilium. Nature. 437:1018-1021. doi:10.1038/nature04117.

Cox, J., and M. Mann. 2008. MaxQuant enables high peptide identification rates, individualized p.p.b.-range mass accuracies and proteome-wide protein quantification. Nat. Biotechnol. 26:1367-1372. doi:10.1038/nbt.1511.

Deutsch, E.W., A. Csordas, Z. Sun, A. Jarnuczak, Y. Perez-Riverol, T. Ternent, D.S. Campbell, M. Bernal-Llinares, S. Okuda, S. Kawano, R.L. Moritz, J.J. Carver, M. Wang, Y. Ishihama, N. Bandeira, H. Hermjakob, and J.A. Vizcaíno. 2017. The ProteomeXchange consortium in 2017: Supporting the cultural change in proteomics public data deposition. Nucleic Acids Res. 45:D1100-D1106. doi:10.1093/nar/gkw936.

Feng, S., Y. Song, M. Shen, S. Xie, W. Li, Y. Lu, Y. Yang, G. Ou, J. Zhou, F. Wang, W. Liu, X. Yan, X. Liang, and T. Zhou. 2017. Microtubule-binding protein FOR20 promotes microtubule depolymerization and cell migration. Cell Discov. 3:1-13. doi:10.1038/celldisc.2017.32.

Goehring, N.W., and A.A. Hyman. 2012. Organelle growth control through limiting pools of cytoplasmic components. Curr. Biol. 22:R330-R339. doi:10.1016/j.cub.2012.03.046.

Gönczy, P. 2015. Centrosomes and cancer: Revisiting a long-standing relationship. Nat. Rev. Cancer. 15:639-652. doi:10.1038/nrc3995.

Hammarsjö, A., Z. Wang, R. Vaz, F. Taylan, M. Sedghi, K.M. Girisha, D. Chitayat, K. Neethukrishna, P. Shannon, R. Godoy, K. Gowrishankar, A. Lindstrand, J. Nasiri, M. Baktashian, P.T. Newton, L. Guo, W. Hofmeister, M. Pettersson, A.S. Chagin, G. Nishimura, L. Yan, N. Matsumoto, A. Nordgren, N. Miyake, G. Grigelioniene, and S. Ikegawa. 2017. Novel KIAA0753 mutations extend the phenotype of skeletal ciliopathies. Sci. Rep. 7. doi:10.1038/s41598-017-15442-1.

Hebbar, M., A. Kanthi, A. Shukla, S. Bielas, and K.M. Girisha. 2018. A biallelic 36-bp insertion in PIBF1 is associated with Joubert syndrome. J. Hum. Genet. 63:935-939. doi:10.1038/s10038-018-0462-7.

Hori, A., and T. Toda. 2017. Regulation of centriolar satellite integrity and its physiology. Cell. Mol. Life Sci. 74:213-229. doi:10.1007/s00018-016-2315-x.

Huangfu, D., A. Liu, A.S. Rakeman, N.S. Murcia, L. Niswander, and K. V. Anderson. 2003. 
Hedgehog signalling in the mouse requires intraflagellar transport proteins. Nature. 426:8387. doi:10.1038/nature02061.

Hunkapiller, J., V. Singla, A. Seol, and J.F. Reiter. 2011. The ciliogenic protein oral-facial-digital 1 regulates the neuronal differentiation of embryonic stem cells. Stem Cells Dev. 20:831841. doi:10.1089/scd.2010.0362.

Ibrahim, R., C. Messaoudi, F.J. Chichon, C. Celati, and S. Marco. 2009. Electron tomography study of isolated human centrioles. Microsc. Res. Tech. 72:42-48. doi:10.1002/jemt.20637.

Jakobsen, L., K. Vanselow, M. Skogs, Y. Toyoda, E. Lundberg, I. Poser, L.G. Falkenby, M. Bennetzen, J. Westendorf, E.A. Nigg, M. Uhlen, A.A. Hyman, and J.S. Andersen. 2011. Novel asymmetrically localizing components of human centrosomes identified by complementary proteomics methods. EMBO J. 30:1520-1535. doi:10.1038/emboj.2011.63.

Keller, L.C., S. Geimer, E. Romijn, J. Yates, I. Zamora, and W.F. Marshall. 2009. Molecular architecture of the centriole proteome: The conserved WD40 domain protein POC1 is required for centriole duplication and length control. Mol. Biol. Cell. 20:1150-1166. doi:10.1091/mbc.E08-06-0619.

Keller, L.C., E.P. Romijn, I. Zamora, J.R. Yates, and W.F. Marshall. 2005. Proteomic analysis of isolated Chlamydomonas centrioles reveals orthologs of ciliary-disease genes. Curr. Biol. 15:1090-1098. doi:10.1016/j.cub.2005.05.024.

Kim, K., K. Lee, and K. Rhee. 2012. CEP90 Is Required for the Assembly and Centrosomal Accumulation of Centriolar Satellites, Which Is Essential for Primary Cilia Formation. PLoS One. 7:e48196. doi:10.1371/journal.pone.0048196.

Kim, K., and K. Rhee. 2011. The pericentriolar satellite protein CEP90 is crucial for integrity of the mitotic spindle pole. J. Cell Sci. 124:338-347. doi:10.1242/jcs.078329.

Kodani, A., T.W. Yu, J.R. Johnson, D. Jayaraman, T.L. Johnson, L. Al-Gazali, L. Sztriha, J.N. Partlow, H. Kim, A.L. Krup, A. Dammermann, N.J. Krogan, C.A. Walsh, and J.F. Reiter. 2015. Centriolar satellites assemble centrosomal microcephaly proteins to recruit CDK2 and promote centriole duplication. Elife. 4:e07519. doi:10.7554/eLife.07519.

Kong, D., N. Sahabandu, C. Sullenberger, A. Vásquez-Limeta, D. Luvsanjav, K. Lukasik, and J. Loncarek. 2020. Prolonged mitosis results in structurally aberrant and over-elongated centrioles. J. Cell Biol. 219. doi:10.1083/jcb.201910019. 
1014

1015

1016

1017

1018

1019

1020

1021

1022

1023

1024

1025

1026

1027

1028

1029

1030

1031

1032

1033

1034

1035

1036

1037

1038

1039

1040

1041

1042

1043

1044

Lu, Q., C. Insinna, C. Ott, J. Stauffer, P.A. Pintado, J. Rahajeng, U. Baxa, V. Walia, A. Cuenca, Y.S. Hwang, I.O. Daar, S. Lopes, J. Lippincott-Schwartz, P.K. Jackson, S. Caplan, and C.J. Westlake. 2015. Early steps in primary cilium assembly require EHD1/EHD3-dependent ciliary vesicle formation. Nat. Cell Biol. 17:228-240. doi:10.1038/ncb3109.

Mahjoub, M.R., Z. Xie, and T. Stearns. 2010. Cep120 is asymmetrically localized to the daughter centriole and is essential for centriole assembly. J. Cell Biol. 191:331-346. doi:10.1083/jcb.201003009.

Marteil, G., A. Guerrero, A.F. Vieira, B.P. De Almeida, P. Machado, S. Mendonça, M. Mesquita, B. Villarreal, I. Fonseca, M.E. Francia, K. Dores, N.P. Martins, S.C. Jana, E.M. Tranfield, N.L. Barbosa-Morais, J. Paredes, D. Pellman, S.A. Godinho, and M. Bettencourt-Dias. 2018. Over-elongation of centrioles in cancer promotes centriole amplification and chromosome missegregation. Nat. Commun. 9:1-17. doi:10.1038/s41467-018-03641-x.

Mazo, G., N. Soplop, W.-J. Wang, K. Uryu, and M.-F. Bryan Tsou. 2016. Spatial Control of Primary Ciliogenesis by Subdistal Appendages Alters Sensation-Associated Properties of Cilia Developmental Cell Spatial Control of Primary Ciliogenesis by Subdistal Appendages Alters Sensation-Associated Properties of Cilia. Dev. Cell. 39:424-437. doi:10.1016/j.devcel.2016.10.006.

McQuin, C., A. Goodman, V. Chernyshev, L. Kamentsky, B.A. Cimini, K.W. Karhohs, M. Doan, L. Ding, S.M. Rafelski, D. Thirstrup, W. Wiegraebe, S. Singh, T. Becker, J.C. Caicedo, and A.E. Carpenter. 2018. CellProfiler 3.0: Next-generation image processing for biology. PLOS Biol. 16:e2005970. doi:10.1371/JOURNAL.PBIO.2005970.

Meier, F., A.D. Brunner, S. Koch, H. Koch, M. Lubeck, M. Krause, N. Goedecke, J. Decker, T. Kosinski, M.A. Park, N. Bache, O. Hoerning, J. Cox, O. Räther, and M. Mann. 2018. Online parallel accumulation-serial fragmentation (PASEF) with a novel trapped ion mobility mass spectrometer. Mol. Cell. Proteomics. 17:2534-2545. doi:10.1074/mcp.TIR118.000900.

Nigg, E.A., and A.J. Holland. 2018. Once and only once: Mechanisms of centriole duplication and their deregulation in diseases. Nat. Rev. Mol. Cell Biol. 19:297-312. doi:10.1038/nrm.2017.127.

Odabasi, E., S. Gul, I.H. Kavakli, and E.N. Firat-Karalar. 2019. Centriolar satellites are required for efficient ciliogenesis and ciliary content regulation. EMBO Rep. 20. doi:10.15252/embr.201947723. 
1045

1046

1047

1048

1049

1050

1051

1052

1053

1054

1055

1056

1057

1058

1059

1060

1061

1062

1063

1064

1065

1066

1067

1068

1069

1070

1071

1072

1073

1074

Paintrand, M., M. Moudjou, H. Delacroix, and M. Bornens. 1992. Centrosome organization and centriole architecture: Their sensitivity to divalent cations. J. Struct. Biol. 108:107-128. doi:10.1016/1047-8477(92)90011-X.

Perez-Riverol, Y., A. Csordas, J. Bai, M. Bernal-Llinares, S. Hewapathirana, D.J. Kundu, A. Inuganti, J. Griss, G. Mayer, M. Eisenacher, E. Pérez, J. Uszkoreit, J. Pfeuffer, T. Sachsenberg, Ş. Yilmaz, S. Tiwary, J. Cox, E. Audain, M. Walzer, A.F. Jarnuczak, T. Ternent, A. Brazma, and J.A. Vizcaíno. 2019. The PRIDE database and related tools and resources in 2019: Improving support for quantification data. Nucleic Acids Res. 47:D442D450. doi:10.1093/nar/gky1106.

Prianichnikov, N., H. Koch, S. Koch, M. Lubeck, R. Heilig, S. Brehmer, R. Fischer, and J. Cox. 2020. Maxquant software for ion mobility enhanced shotgun proteomics. Mol. Cell. Proteomics. 19:1058-1069. doi:10.1074/mcp.TIR119.001720.

Prosser, S.L., and L. Pelletier. 2020. Centriolar satellite biogenesis and function in vertebrate cells. J. Cell Sci. 133. doi:10.1242/jcs.239566.

Reiter, J.F., and M.R. Leroux. 2017. Genes and molecular pathways underpinning ciliopathies. Nat. Rev. Mol. Cell Biol. 18:533-547. doi:10.1038/nrm.2017.60.

Schmidt, K.N., S. Kuhns, A. Neuner, B. Hub, H. Zentgraf, and G. Pereira. 2012a. Cep164 mediates vesicular docking to the mother centriole during early steps of ciliogenesis. J. Cell Biol. 199:1083-1101. doi:10.1083/jcb.201202126.

Schmidt, K.N., S. Kuhns, A. Neuner, B. Hub, H. Zentgraf, and G. Pereira. 2012b. Cep164 mediates vesicular docking to the mother centriole during early steps of ciliogenesis. J. Cell Biol. 199:1083-1101. doi:10.1083/jcb.201202126.

Schneider, C.A., W.S. Rasband, and K.W. Eliceiri. 2012. NIH Image to ImageJ: 25 years of image analysis. Nat. Methods. 9:671-675. doi:10.1038/nmeth.2089.

Shen, Y., H. Wang, Z. Liu, M. Luo, S. Ma, C. Lu, Z. Cao, Y. Yu, R. Cai, C. Chen, Q. Li, H. Gao, Y. Peng, B. Xu, and X. Ma. 2020. Identification of two novel pathogenic variants of PIBF1 by whole exome sequencing in a 2-year-old boy with Joubert syndrome. BMC Med. Genet. 21:192. doi:10.1186/s12881-020-01130-x.

Shi, X., G. Garcia, J.C. Van De Weghe, R. McGorty, G.J. Pazour, D. Doherty, B. Huang, and J.F. Reiter. 2017. Super-resolution microscopy reveals that disruption of ciliary transition-zone 
architecture causes Joubert syndrome. Nat. Cell Biol. 19:1178-1188. doi:10.1038/ncb3599.

1076

1077

1078

1079

1080

1081

1082

1083

1084

1085

1086

1087

1088

1089

1090

1091

1092

1093

1094

1095

1096

1097

1098

1099

1100

1101

1102

1103

1104

Shi, X., Q. Li, Z. Dai, A. Tran, S. Feng, A.D. Ramirez, Z. Lin, X. Wang, T.T. Chow, I.B. Seiple, and B. Huang. 2019. Label-retention expansion microscopy. bioRxiv. 687954. doi:10.1101/687954.

Sillibourne, J.E., I. Hurbain, T. Grand-Perret, B. Goud, P. Tran, and M. Bornens. 2013. Primary ciliogenesis requires the distal appendage component Cep123. Biol. Open. 2:535-545. doi:10.1242/bio.20134457.

Singla, V., M. Romaguera-Ros, J.M. Garcia-Verdugo, and J.F. Reiter. 2010. Ofd1, a Human Disease Gene, Regulates the Length and Distal Structure of Centrioles. Dev. Cell. 18:410424. doi:10.1016/j.devcel.2009.12.022.

Srivastava, S., and D. Panda. 2017. A centrosomal protein FOR20 regulates microtubule assembly dynamics and plays a role in cell migration. Biochem. J. 474:2841-2859. doi:10.1042/BCJ20170303.

Stephen, J., T. Vilboux, L. Mian, C. Kuptanon, C.M. Sinclair, D. Yildirimli, D.M. Maynard, J. Bryant, R. Fischer, M. Vemulapalli, J.C. Mullikin, M. Huizing, W.A. Gahl, M.C. V. Malicdan, and M. Gunay-Aygun. 2017. Mutations in KIAA0753 cause Joubert syndrome associated with growth hormone deficiency. Hum. Genet. 136:399-408. doi:10.1007/s00439-017-1765-z.

Tanos, B.E., H.J. Yang, R. Soni, W.J. Wang, F.P. Macaluso, J.M. Asara, and M.F.B. Tsou. 2013. Centriole distal appendages promote membrane docking, leading to cilia initiation. Genes Dev. 27:163-168. doi:10.1101/gad.207043.112.

Teo, G., G. Liu, J. Zhang, A.I. Nesvizhskii, A.C. Gingras, and H. Choi. 2014. SAINTexpress: Improvements and additional features in Significance Analysis of INTeractome software. J. Proteomics. 100:37-43. doi:10.1016/j.jprot.2013.10.023.

Thauvin-Robinet, C., J.S. Lee, E. Lopez, V. Herranz-Pérez, T. Shida, B. Franco, L. Jego, F. Ye, L. Pasquier, P. Loget, N. Gigot, B. Aral, C.A.M. Lopes, J. St-Onge, A.L. Bruel, J. Thevenon, S. González-Granero, C. Alby, A. Munnich, M. Vekemans, F. Huet, A.M. Fry, S. Saunier, J.B. Rivière, T. Attié-Bitach, J.M. Garcia-Verdugo, L. Faivre, A. Mégarbané, and M. V. Nachury. 2014. The oral-facial-digital syndrome gene C2CD3 encodes a positive regulator of centriole elongation. Nat. Genet. 46:905-911. doi:10.1038/ng.3031.

Tsai, J.J., W. Bin Hsu, J.H. Liu, C.W. Chang, and T.K. Tang. 2019. CEP120 interacts with C2CD3 
and Talpid3 and is required for centriole appendage assembly and ciliogenesis. Sci. Rep. 9:1-15. doi:10.1038/s41598-019-42577-0.

Viol, L., S. Hata, A. Pastor-Peidro, A. Neuner, F. Murke, P. Wuchter, A.D. Ho, B. Giebel, and G. Pereira. 2020. Nek2 kinase displaces distal appendages from the mother centriole prior to mitosis. J. Cell Biol. 219. doi:10.1083/jcb.201907136.

Wang, L., M. Failler, W. Fu, and B.D. Dynlacht. 2018. A distal centriolar protein network controls

Wang, L., K. Lee, R. Malonis, I. Sanchez, and B.D. Dynlacht. 2016. Tethering of an E3 ligase by PCM1 regulates the abundance of centrosomal KIAA0586/Talpid3 and promotes ciliogenesis. Elife. 5. doi:10.7554/eLife.12950.

Wassie, A.T., Y. Zhao, and E.S. Boyden. 2019. Expansion microscopy: principles and uses in biological research. Nat. Methods. 16:33-41. doi:10.1038/s41592-018-0219-4.

Westlake, C.J., L.M. Baye, M. V. Nachury, K.J. Wright, K.E. Ervin, L. Phu, C. Chalouni, J.S. Beck,

Wheway, G., M. Schmidts, D.A. Mans, K. Szymanska, T.-M.T. Nguyen, H. Racher, I.G. Phelps, G. Toedt, J. Kennedy, K.A. Wunderlich, N. Sorusch, Z.A. Abdelhamed, S. Natarajan, W. Herridge, J. van Reeuwijk, N. Horn, K. Boldt, D.A. Parry, S.J.F. Letteboer, S. Roosing, M. Adams, S.M. Bell, J. Bond, J. Higgins, E.E. Morrison, D.C. Tomlinson, G.G. Slaats, T.J.P. van Dam, L. Huang, K. Kessler, A. GiessI, C. V. Logan, E.A. Boyle, J. Shendure, S. Anazi, M. Aldahmesh, S. Al Hazzaa, R.A. Hegele, C. Ober, P. Frosk, A.A. Mhanni, B.N. Chodirker, A.E. Chudley, R. Lamont, F.P. Bernier, C.L. Beaulieu, P. Gordon, R.T. Pon, C. Donahue, A.J. Barkovich, L. Wolf, C. Toomes, C.T. Thiel, K.M. Boycott, M. McKibbin, C.F. Inglehearn, F. Stewart, H. Omran, M.A. Huynen, P.I. Sergouniotis, F.S. Alkuraya, J.S. Parboosingh, A.M. Innes, C.E. Willoughby, R.H. Giles, A.R. Webster, M. Ueffing, O. Blacque, J.G. Gleeson, U. Wolfrum, P.L. Beales, T. Gibson, D. Doherty, H.M. Mitchison, R. Roepman, and C.A. Johnson. 2015. An siRNA-based functional genomics screen for the identification of regulators of ciliogenesis and ciliopathy genes. Nat. Cell Biol. 17:1074-1087. doi:10.1038/ncb3201. 
Winey, M., and E. O'Toole. 2014. Centriole structure. Philos. Trans. R. Soc. B Biol. Sci. 369. doi:10.1098/rstb.2013.0457.

Wu, C.T., H.Y. Chen, and T.K. Tang. 2018. Myosin-Va is required for preciliary vesicle transportation to the mother centriole during ciliogenesis. Nat. Cell Biol. 20:175-185. doi:10.1038/s41556-017-0018-7.

Yang, T.T., W.M. Chong, W.J. Wang, G. Mazo, B. Tanos, Z. Chen, T.M.N. Tran, Y. De Chen, R.R. Weng, C.E. Huang, W.N. Jane, M.F.B. Tsou, and J.C. Liao. 2018. Super-resolution architecture of mammalian centriole distal appendages reveals distinct blade and matrix functional components. Nat. Commun. 9:1-11. doi:10.1038/s41467-018-04469-1.

Ye, X., H. Zeng, G. Ning, J.F. Reiter, and A. Liu. 2014. C2cd3 is critical for centriolar distal appendage assembly and ciliary vesicle docking in mammals. Proc. Natl. Acad. Sci. U. S. A. 111:2164-2169. doi:10.1073/pnas.1318737111.

Zimmermann, L., A. Stephens, S.Z. Nam, D. Rau, J. Kübler, M. Lozajic, F. Gabler, J. Söding, A.N. Lupas, and V. Alva. 2018. A Completely Reimplemented MPI Bioinformatics Toolkit with a New HHpred Server at its Core. J. Mol. Biol. 430:2237-2243. doi:10.1016/j.jmb.2017.12.007. 
bioRxiv preprint doi: https://doi.org/10.1101/2021.03.01.433418; this version posted June 10, 2021. The copyright holder for this preprint

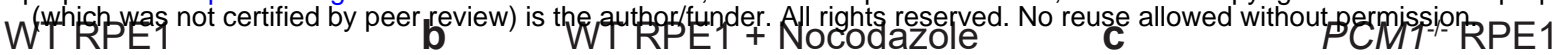
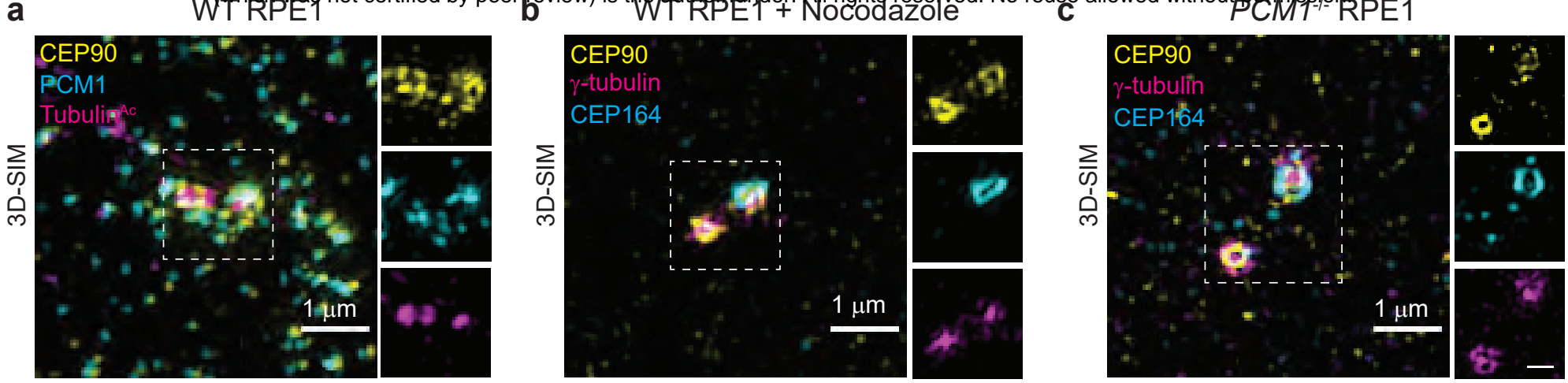

d

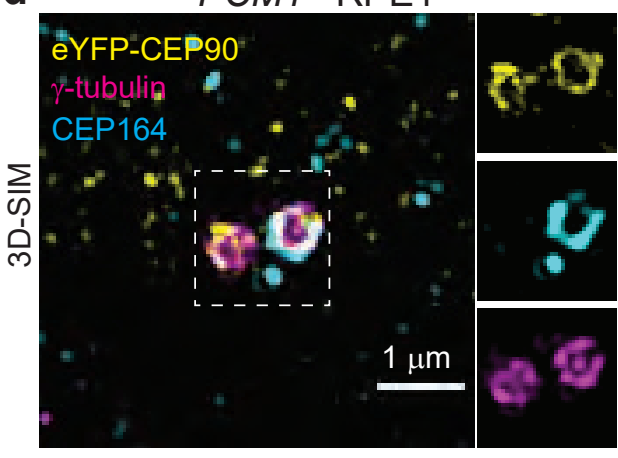

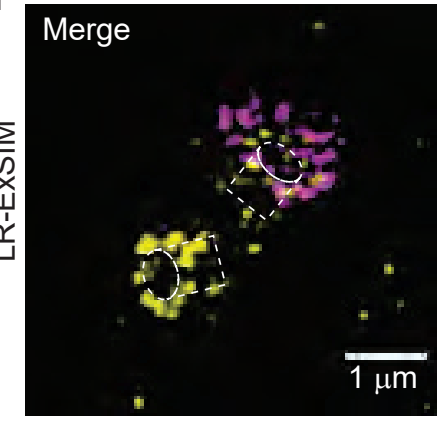

g

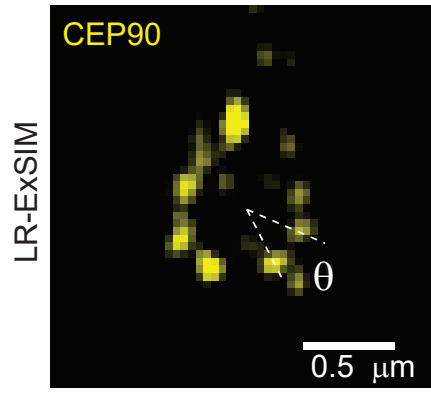

\section{CEP90}

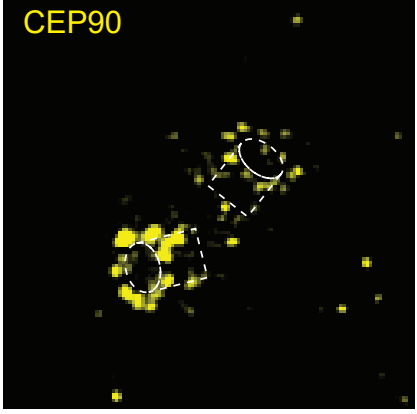

h

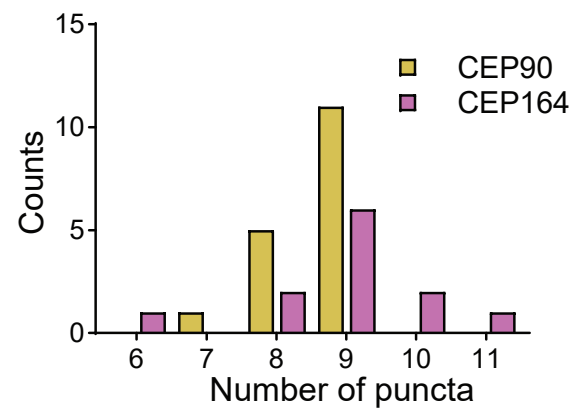

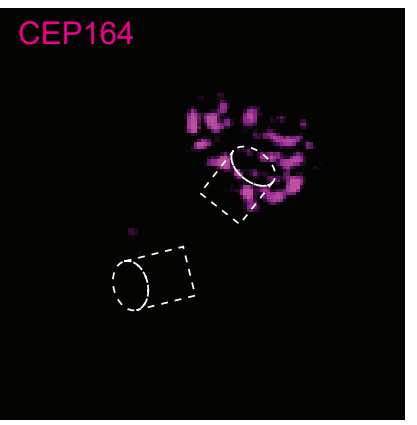

i

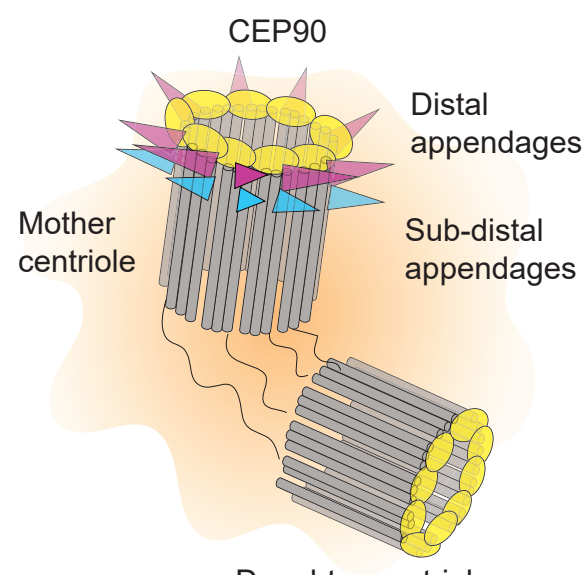

Daughter centriole

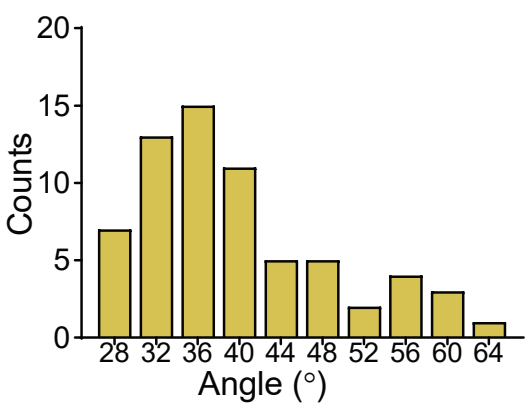


bioRxiv preprint doi: https://doi.org/10.1101/2021.03.01.433418; this version posted June 10, 2021. The copyright holder for this preprint (which was not certified by peer review) is the author/funder. All rights reserved. No reuse allowed without permission.

a

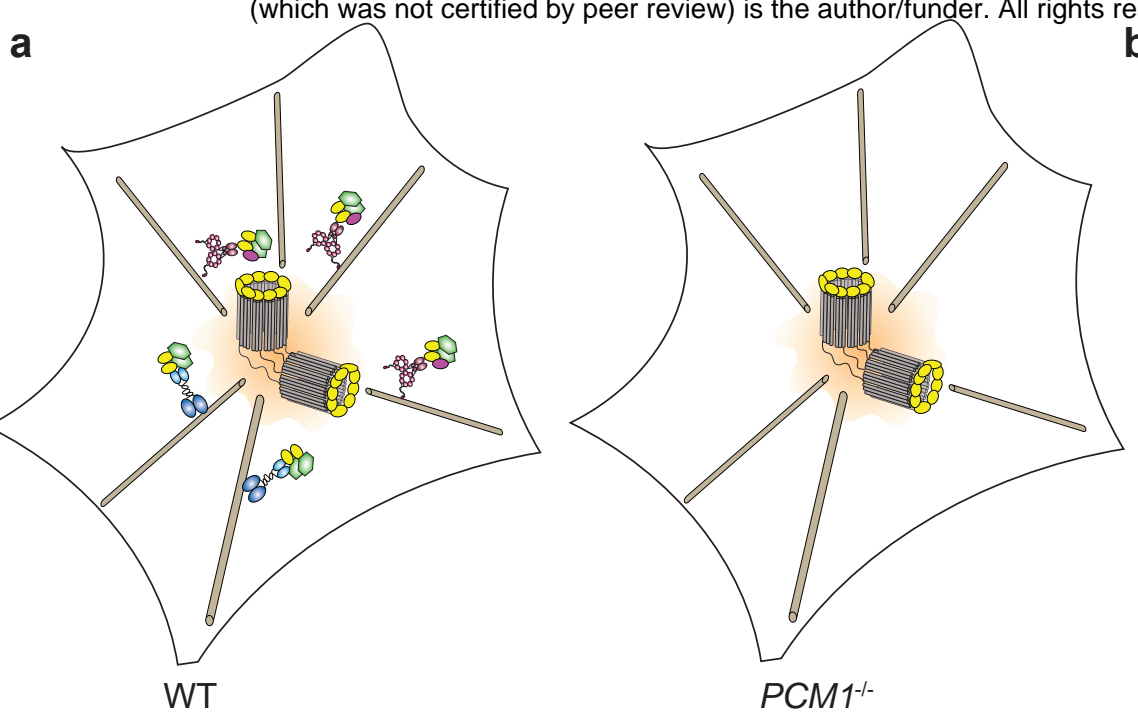

Independent cell lines expressing GFP (control) or eYFP-CEP90

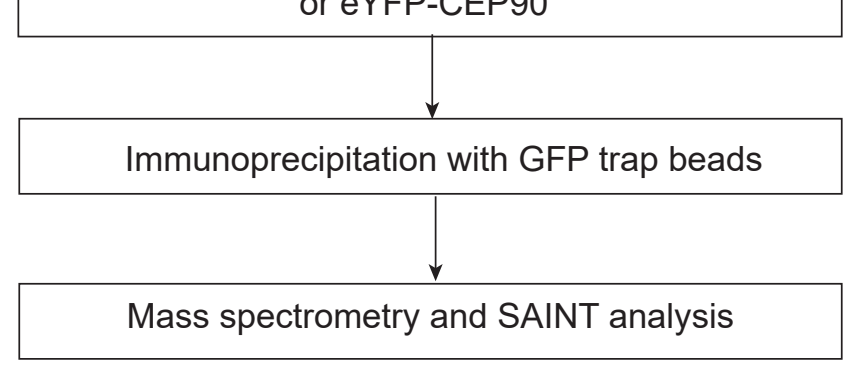

C

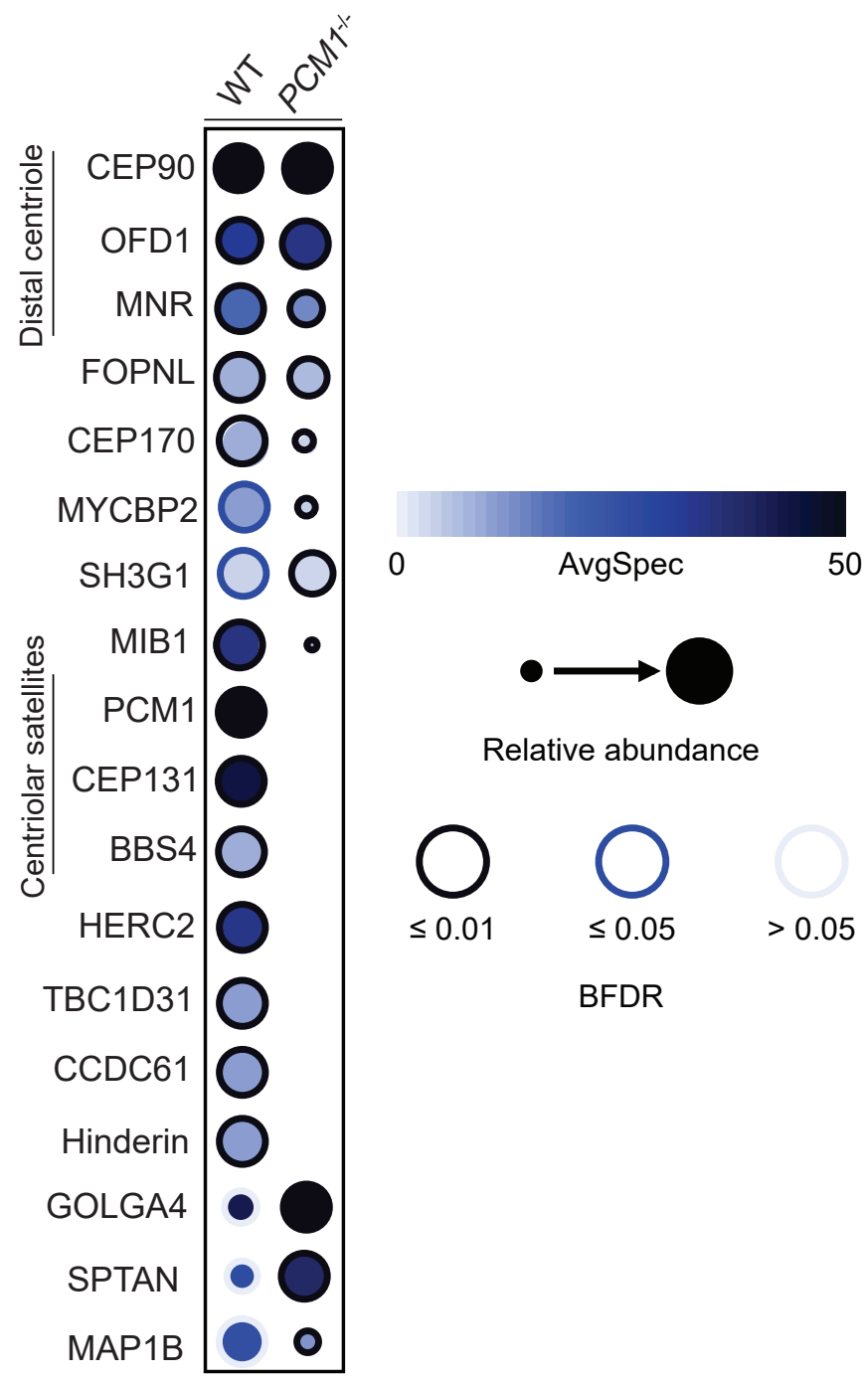

b

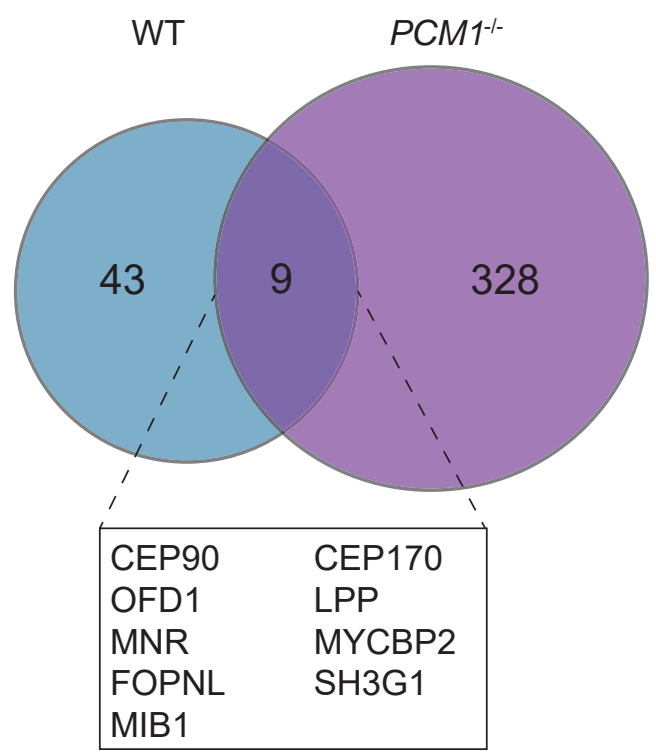

d

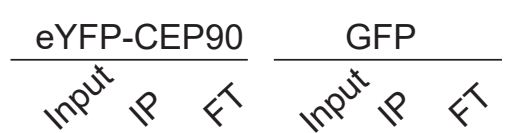

Coomassie

eYFP-CEP90
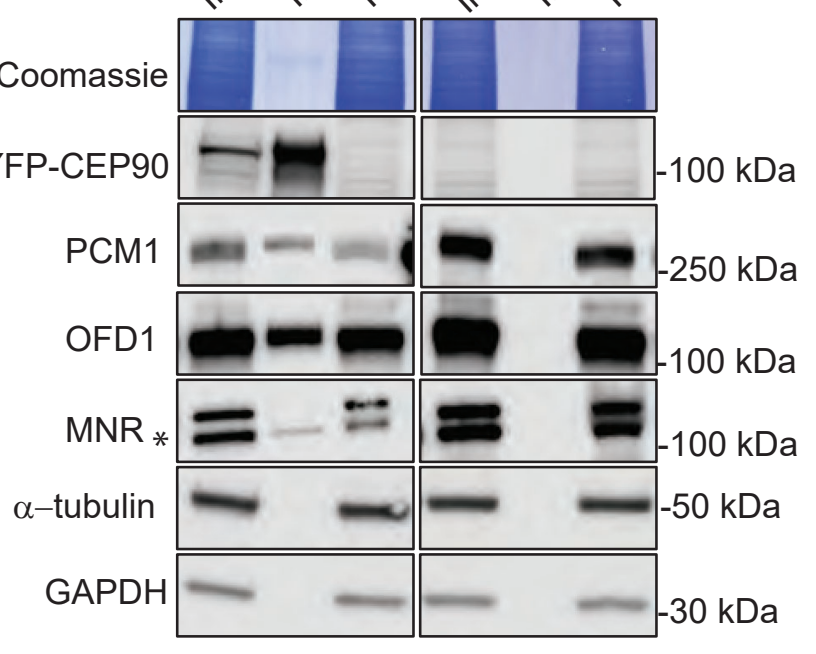

e

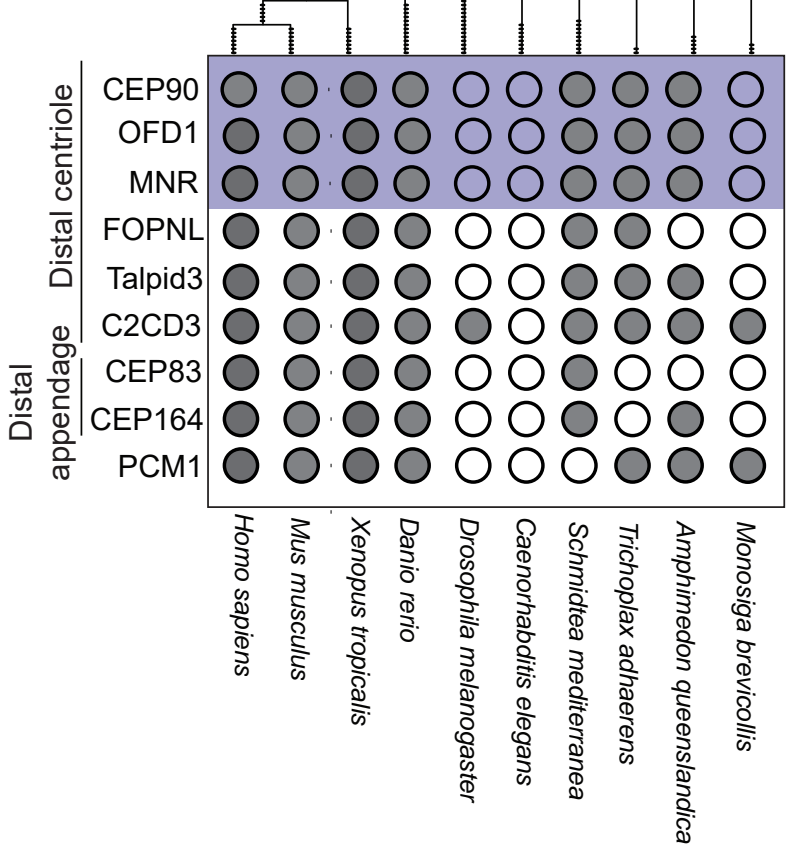


bioRxiv preprint doi: https://doi.org/10.1101/2021.03.01.4334/8; this version posted June 1 [Q, 2021 . The copyright holder for this preprint

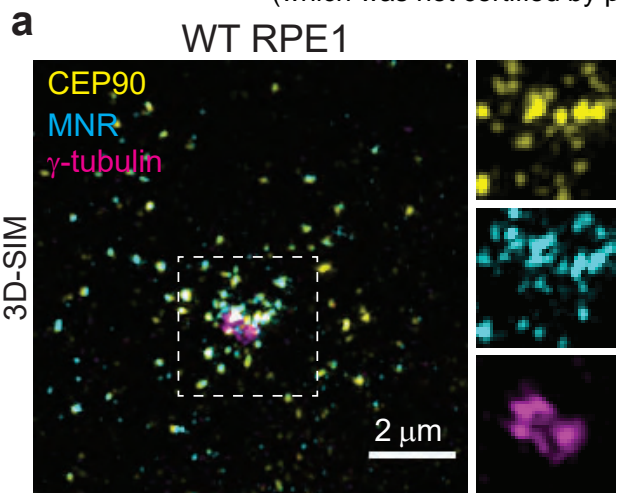

C

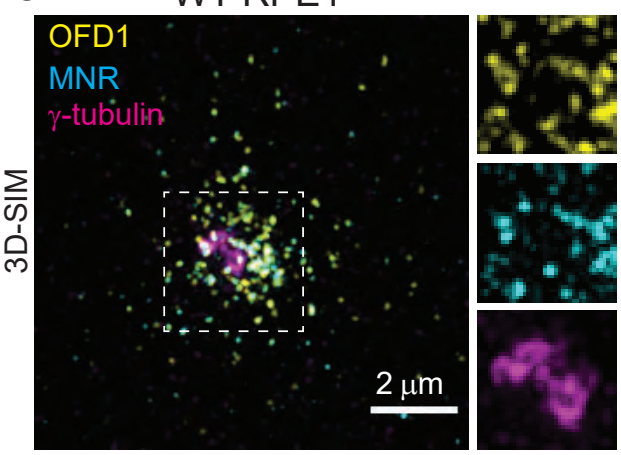

g b

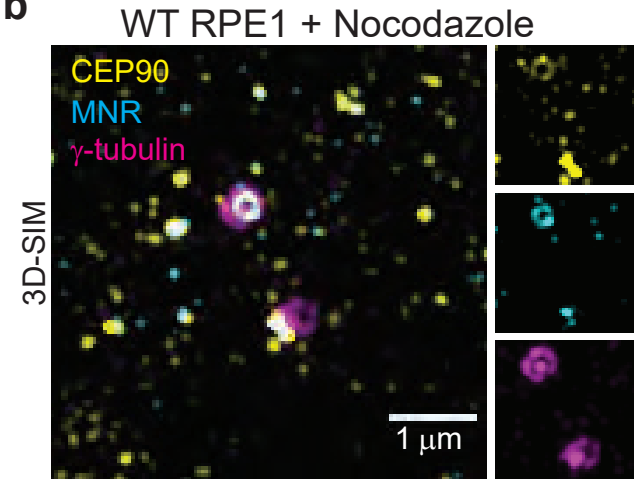

d WT RPE1 + Nocodazole

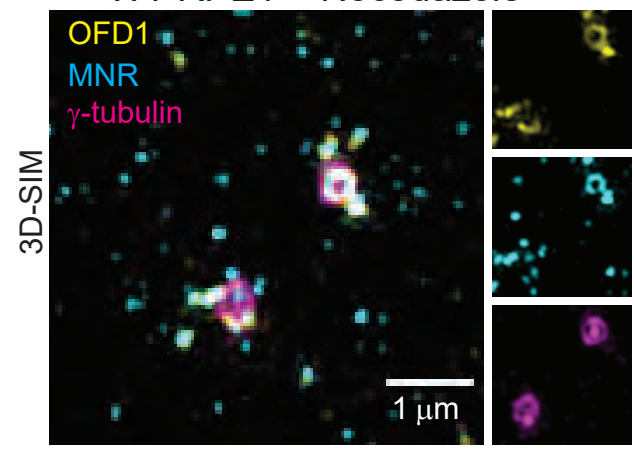

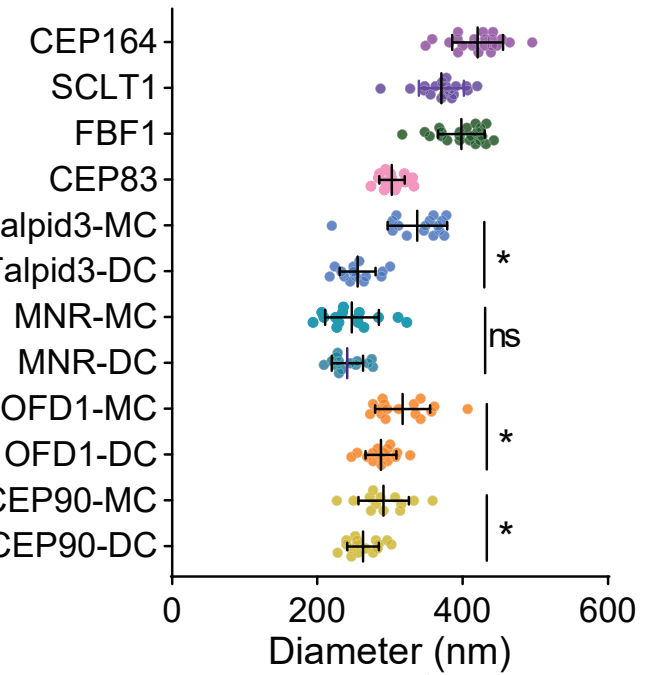

f

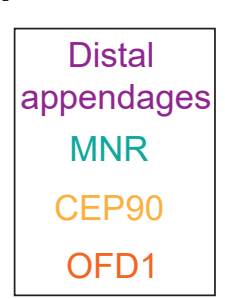

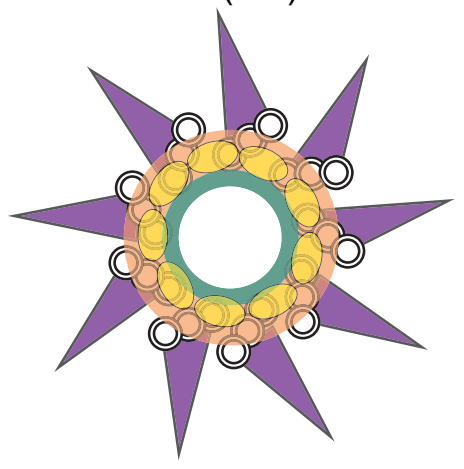

EdU-

CENPF-
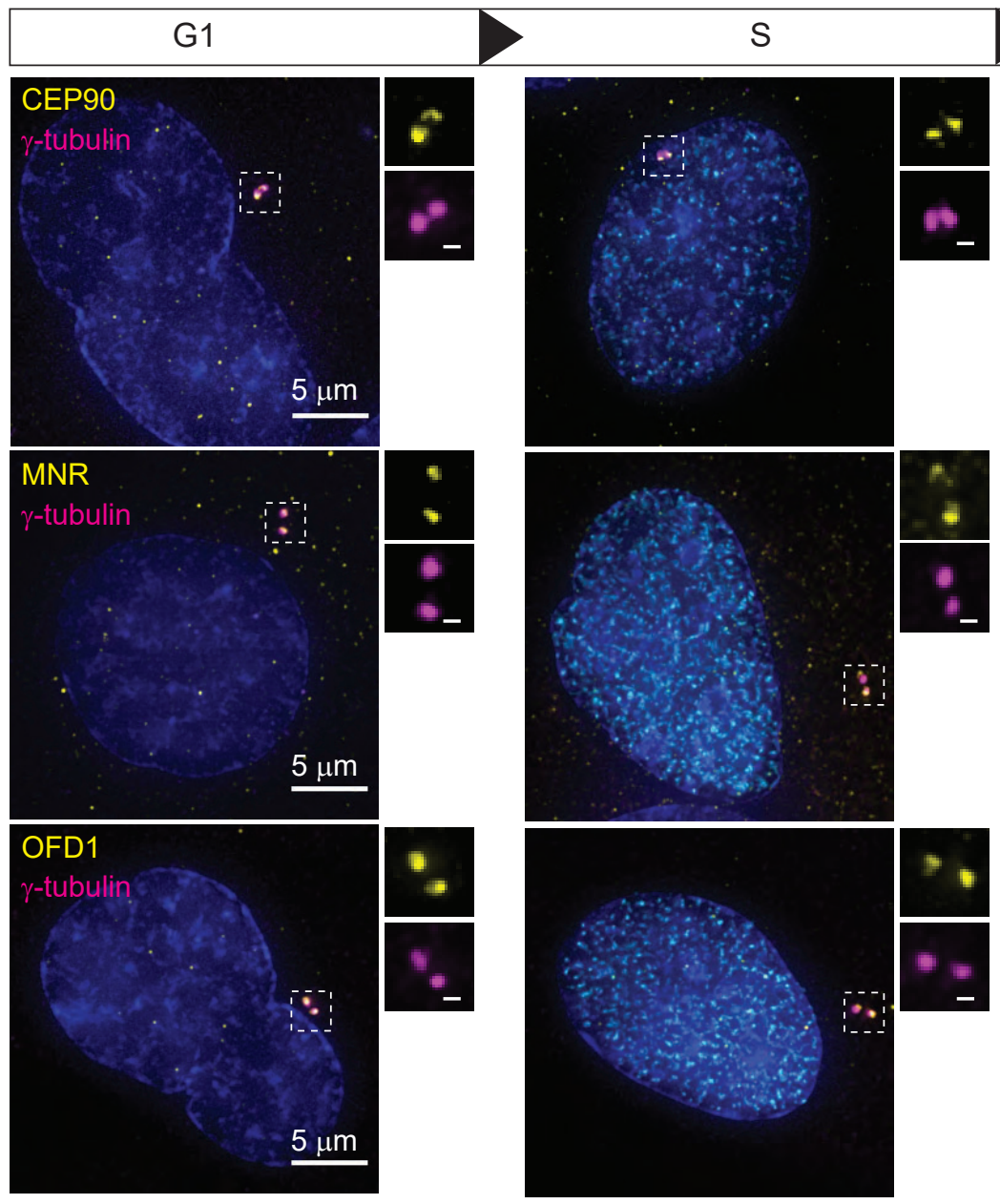

CENPF+ 


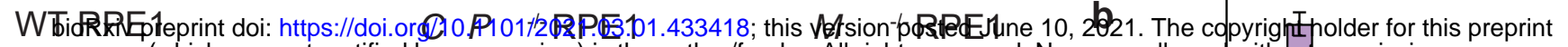
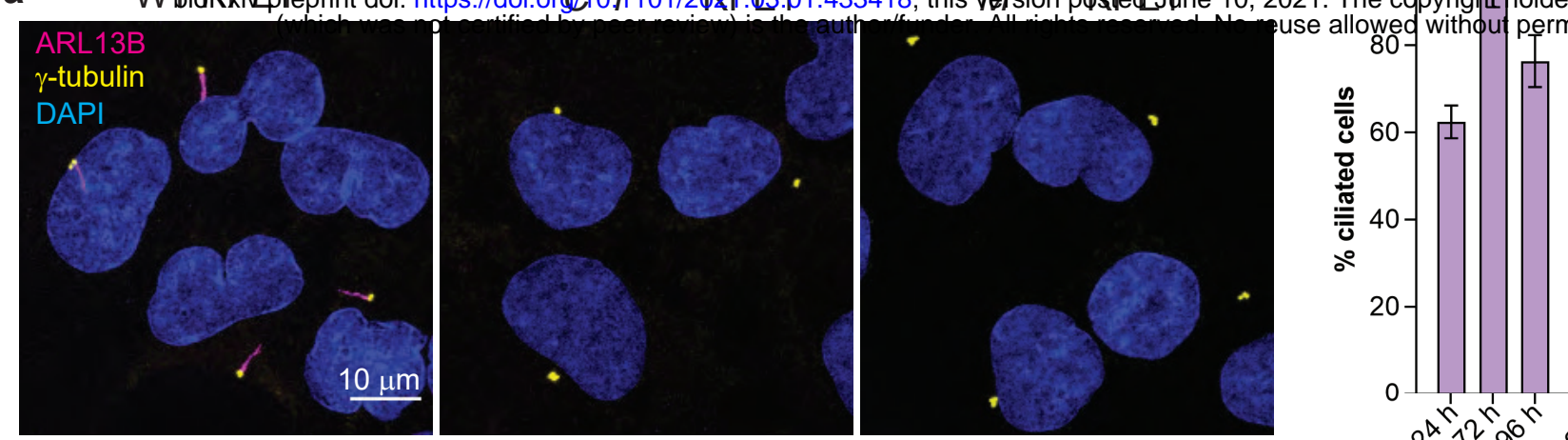

c

C Control

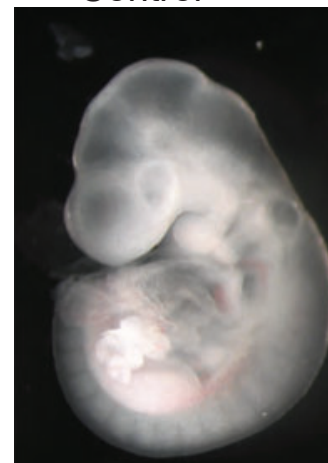

E9.5

e

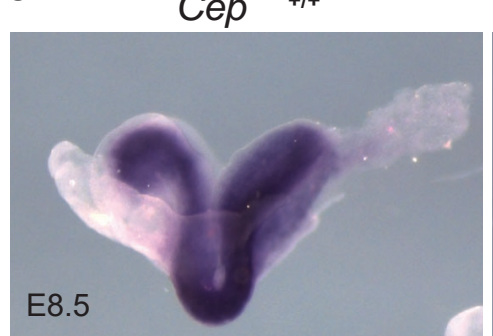

g

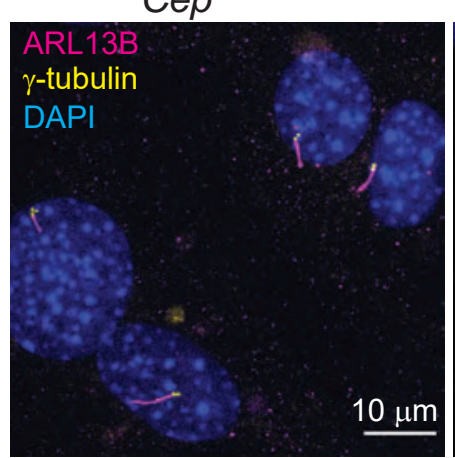

j

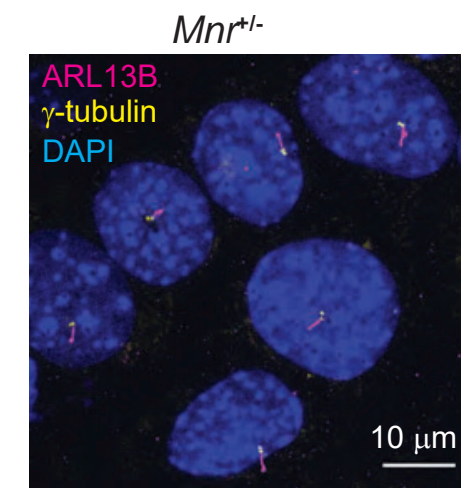

Mnr'-
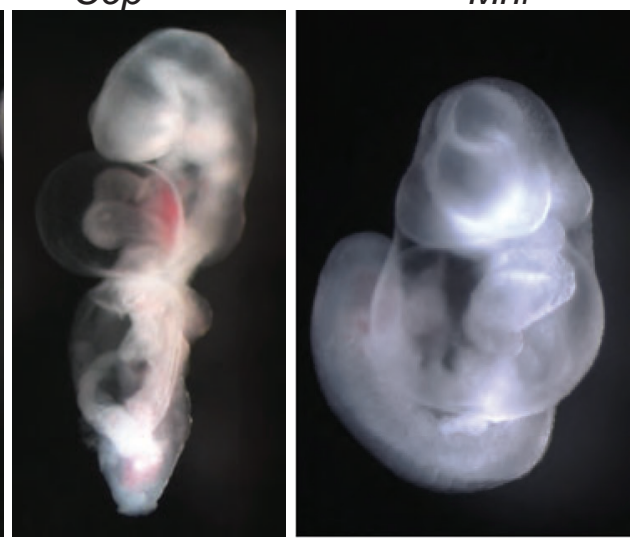

Cep

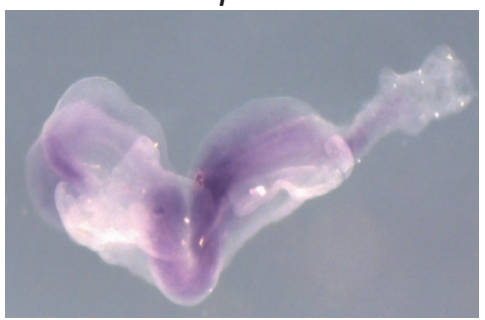

Cep

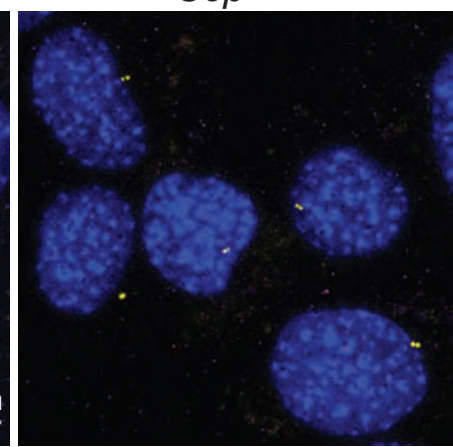

Mnr ${ }^{-}$

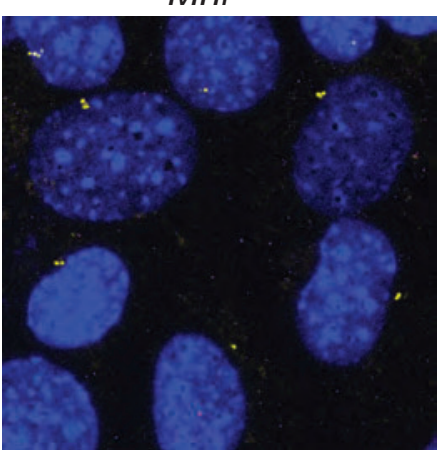

d

Cep

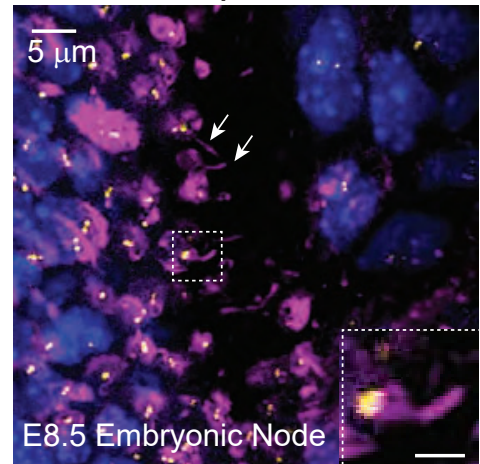

$\mathrm{Mnr}^{+/-}$

f

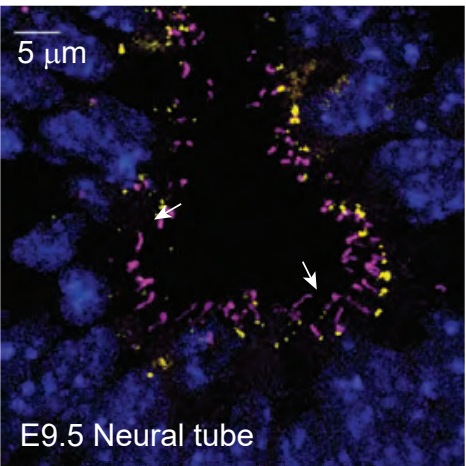

Mnr ${ }^{\mathrm{l}}$

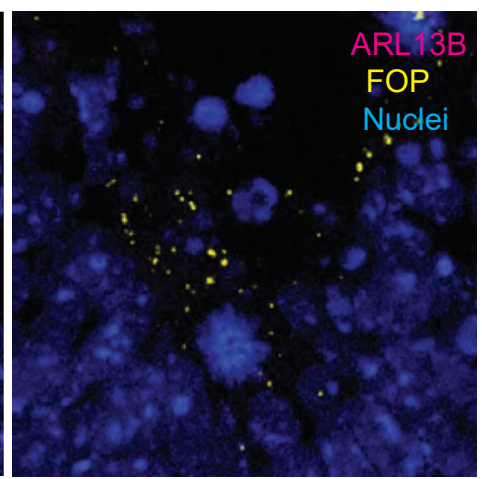

h
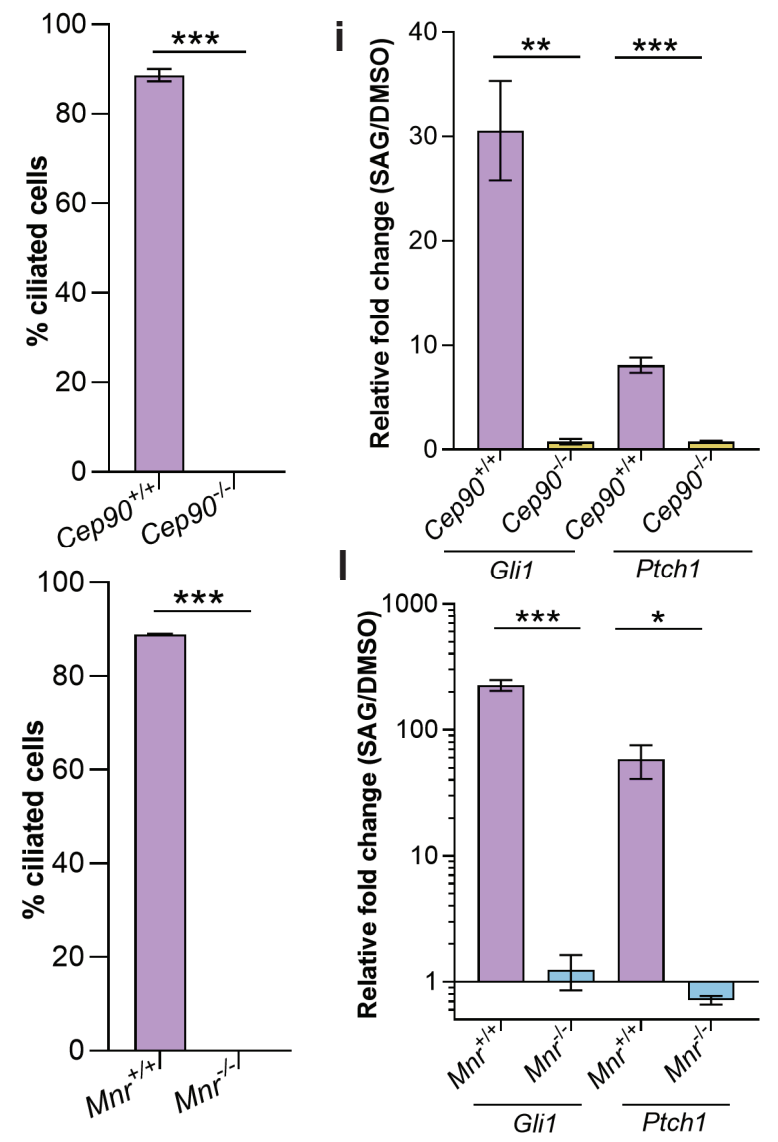
bioRxiv preprint doi: https://doi.org/10.1101/2021.03.01.433418; this version posted June 10, 2021. The copyright holder for this preprint

a

FL-CEP90

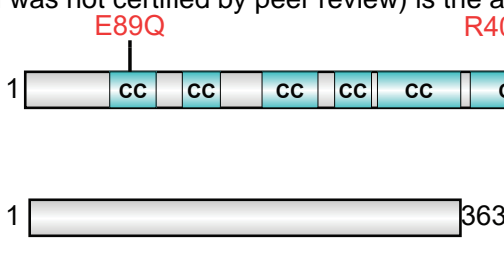

CEP90 $363-757$

$$
363
$$
$405 Q$ Q D637A

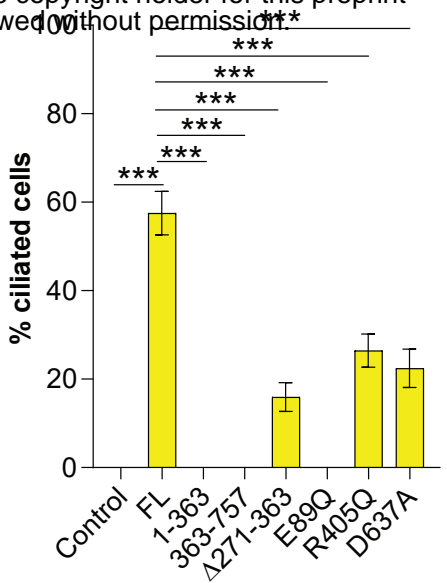

C

CEP90 $4271-363$
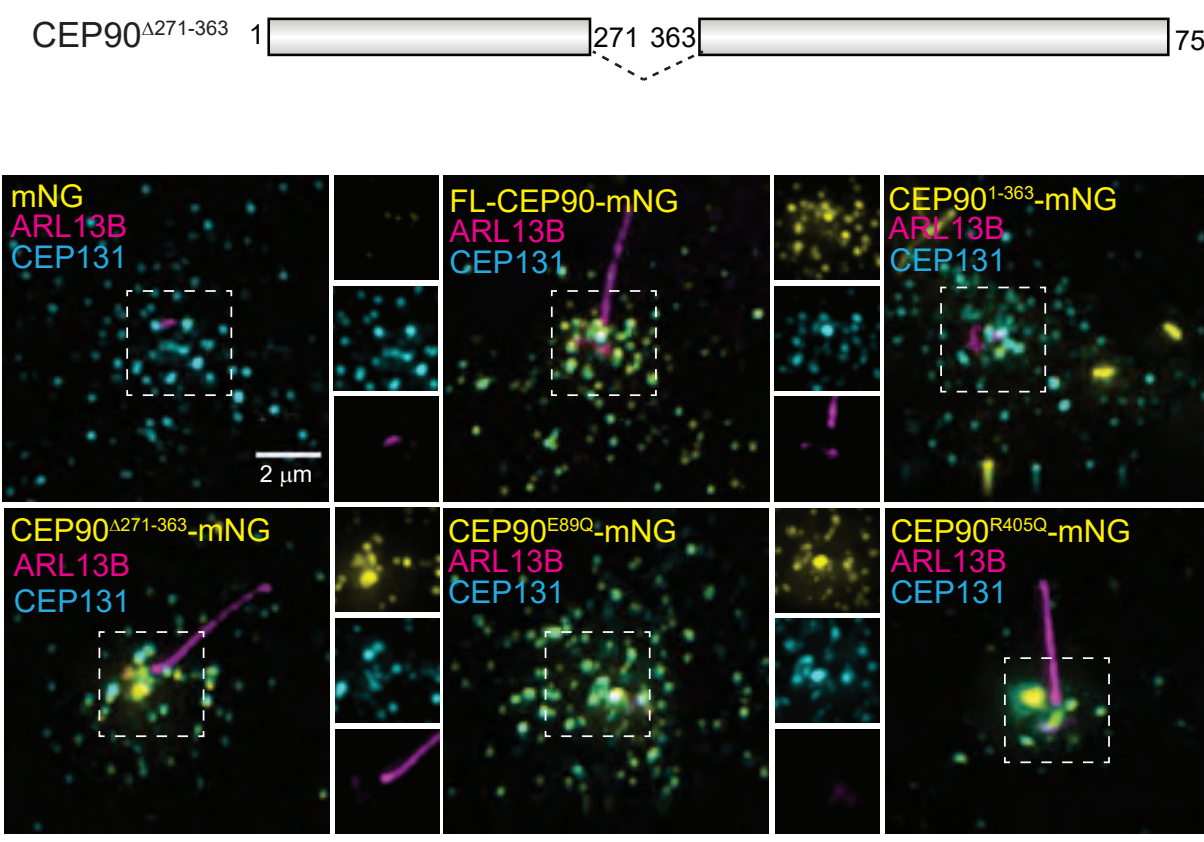

d

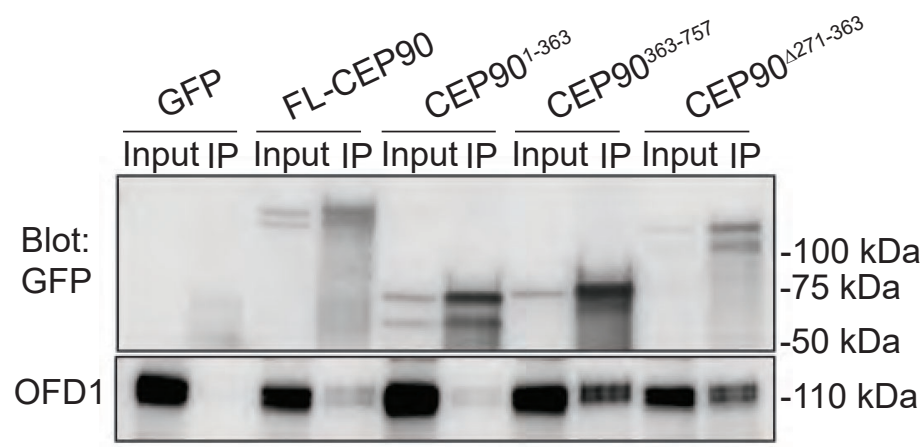

f

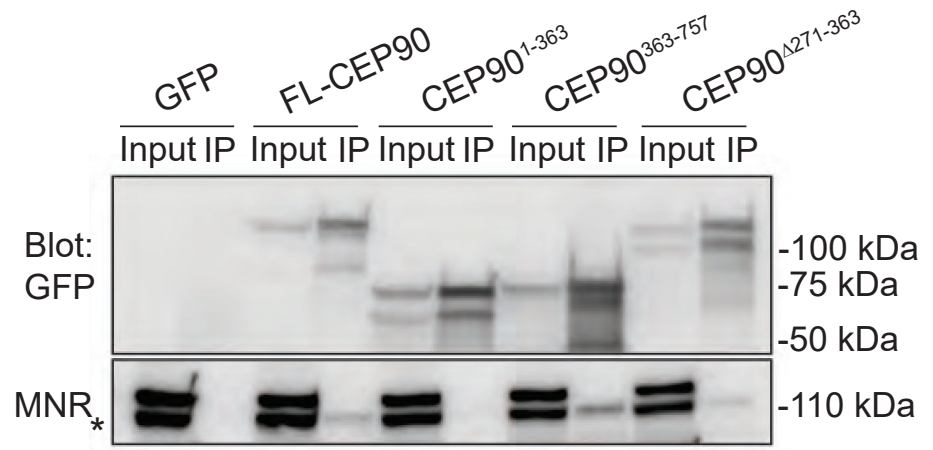

g
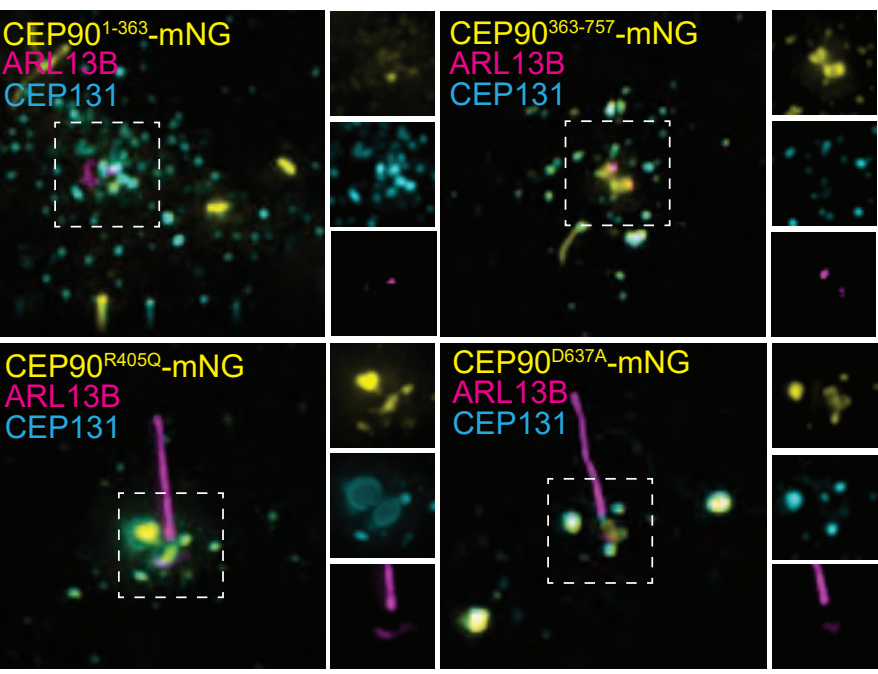

e

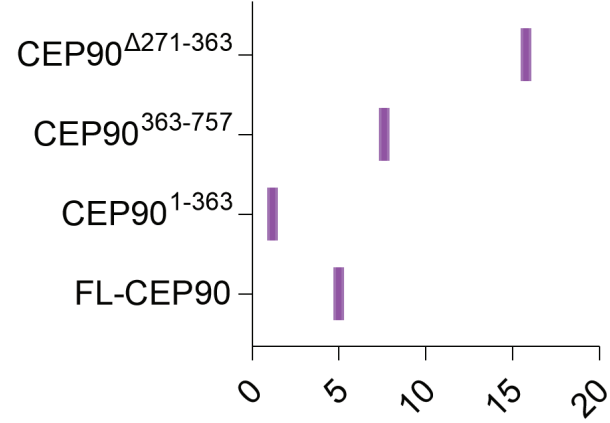

OFD1 IP/GFP input ratio

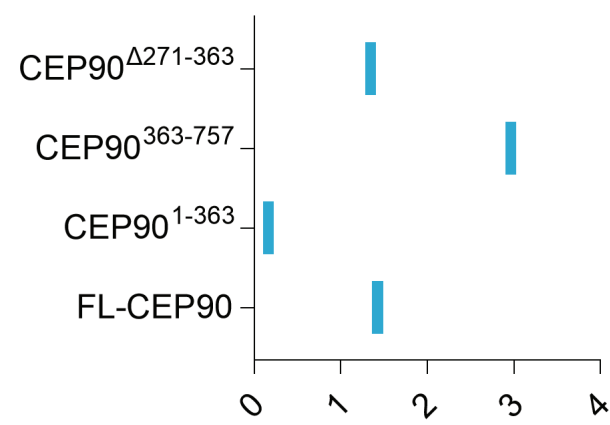

MNR IP/GFP input ratio 
bioRxiv preprint doi: https://doi.org/10.1101/2021.03.01.433418; this version posted June 10, 2021. The Gopyrigh巨 holder for this preprint
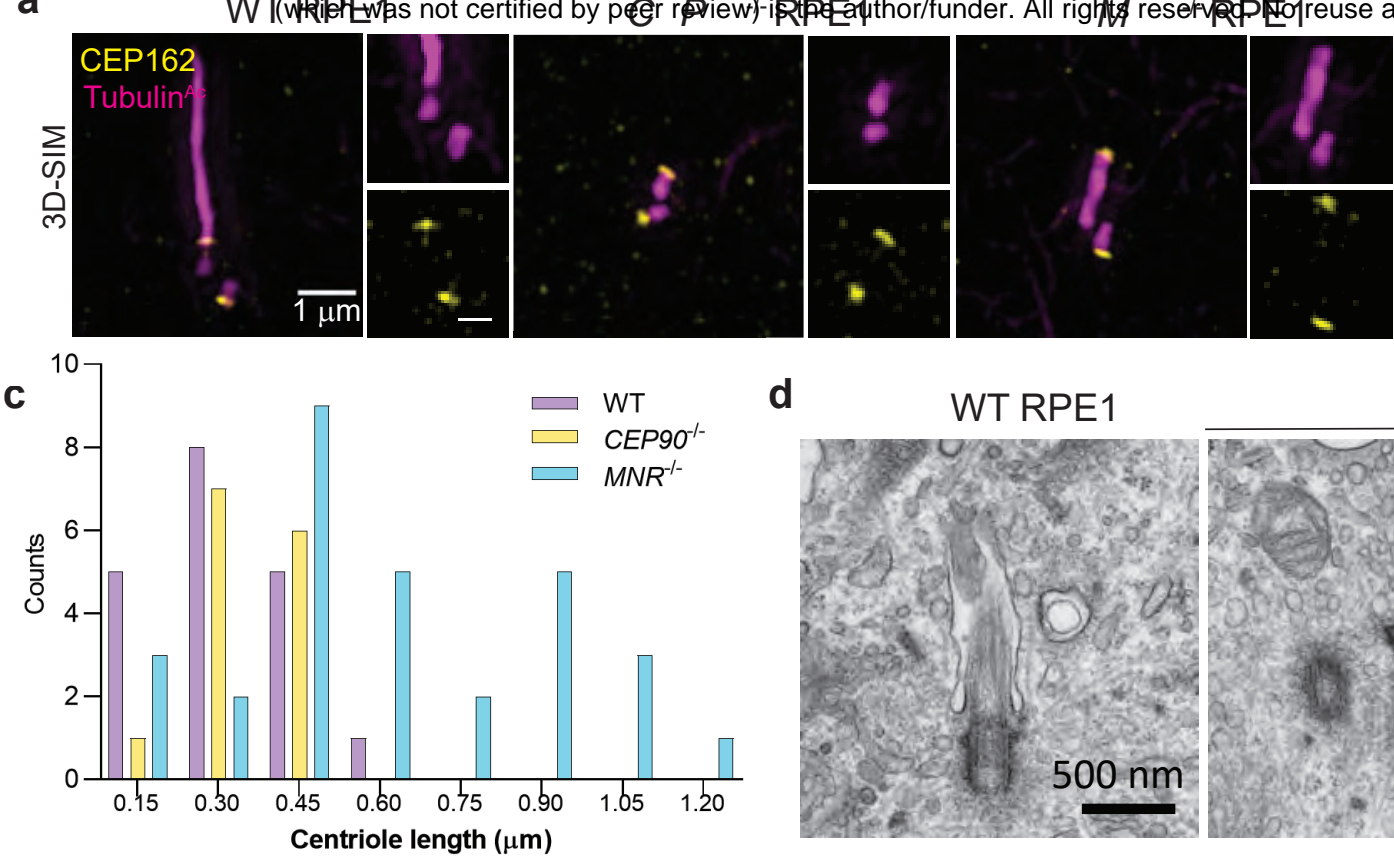

d
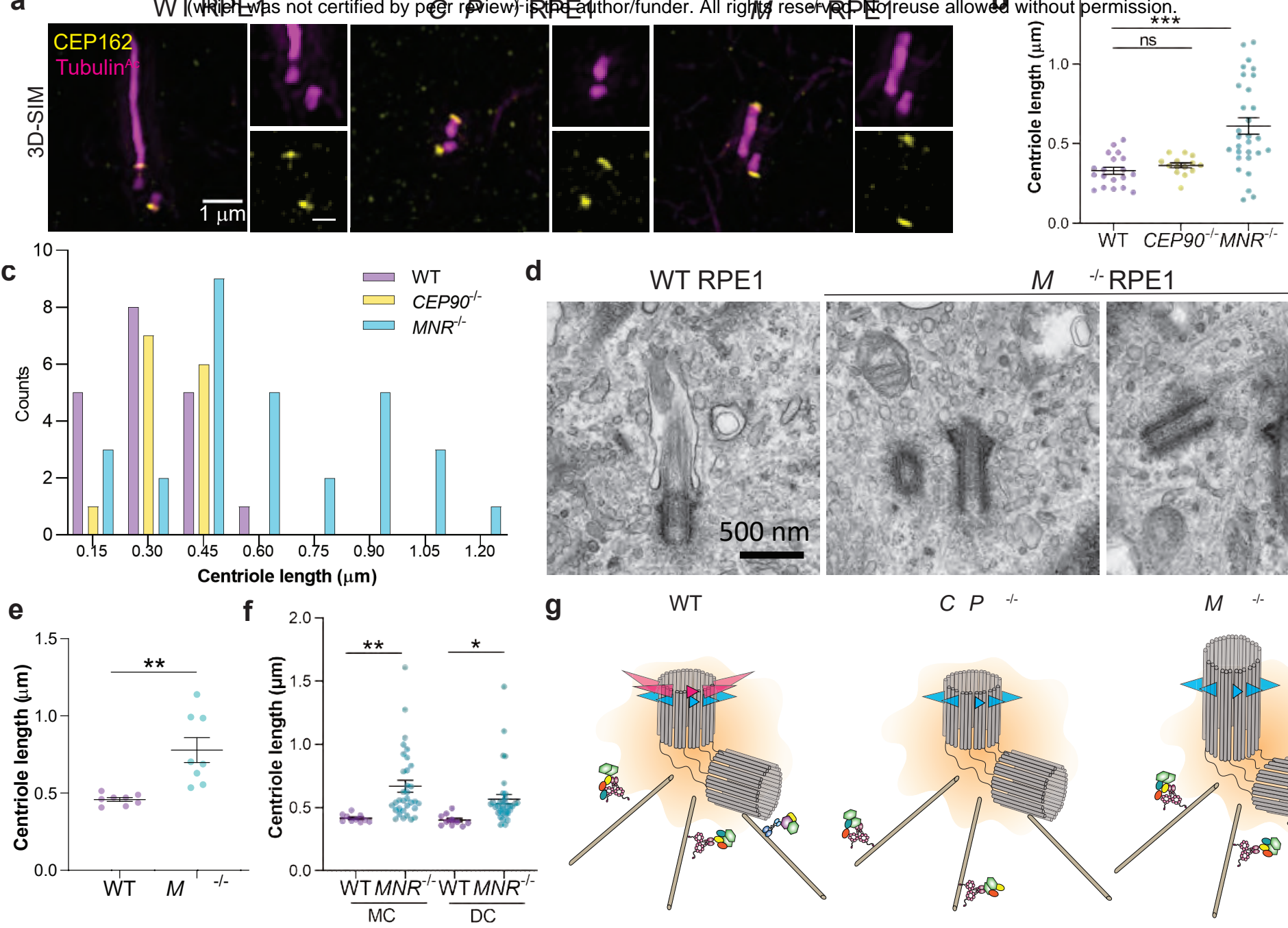

g

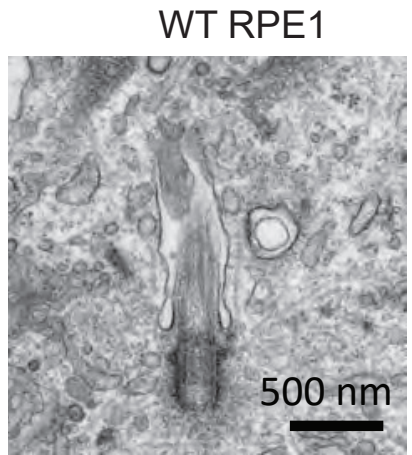

$M \quad$-RPE1

h

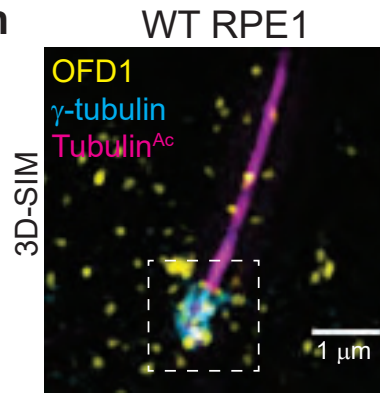

C $P$ - ${ }^{-R P E} 1$
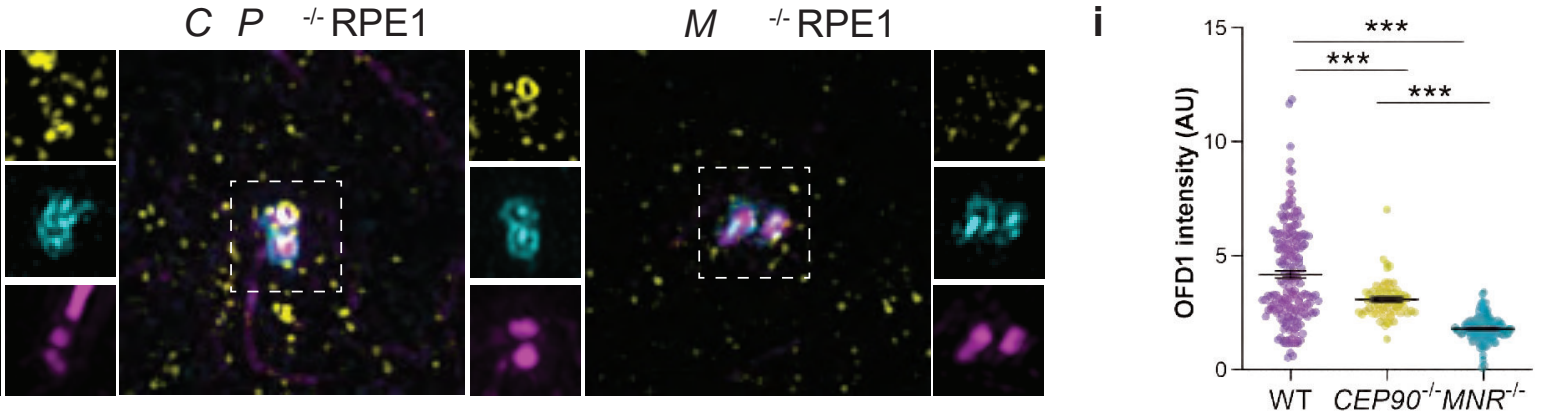


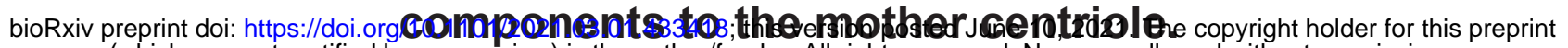

(which was not certified by peer review) is the author/funder. All rights reserved. No reuse allowed without permission.

a WT RPE1

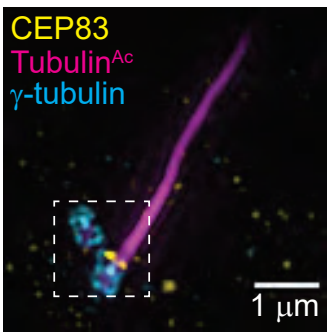

C

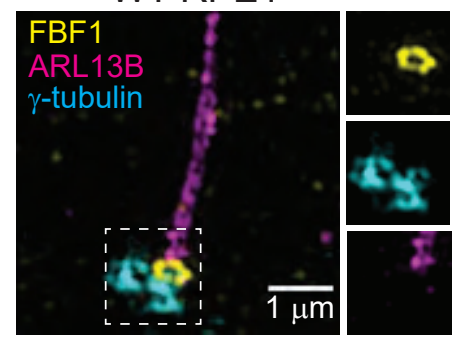

e

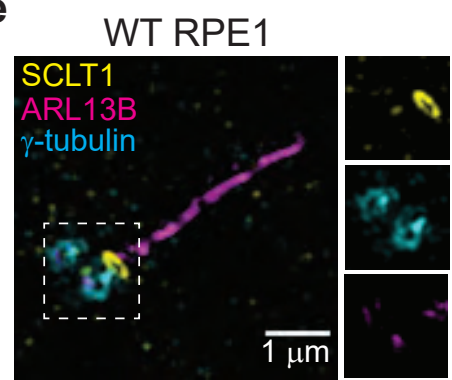

g

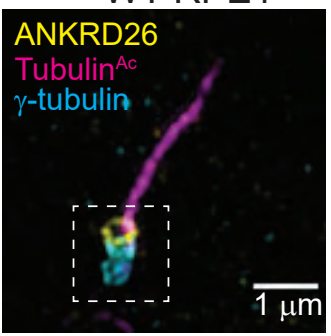

i
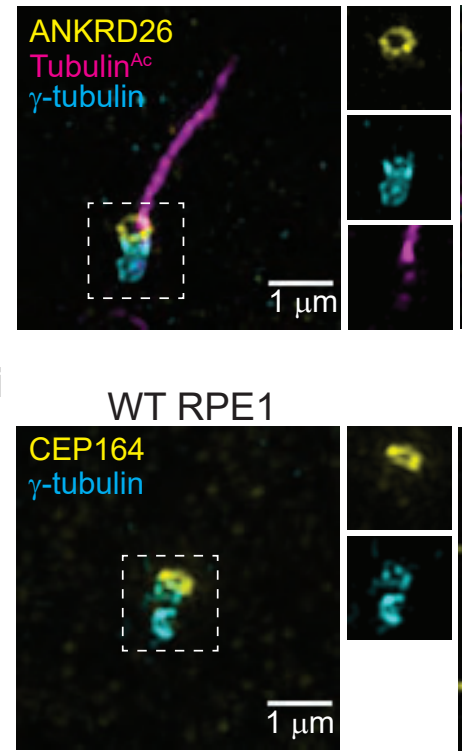

C $P \quad$ - RPE1
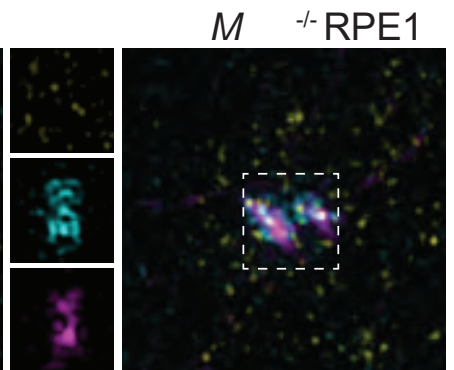

C $P$ P $\quad$ - RPE 1

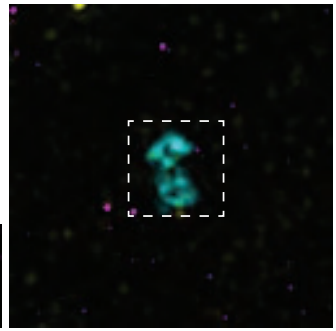

C $P$ - ${ }^{-/}$RPE1
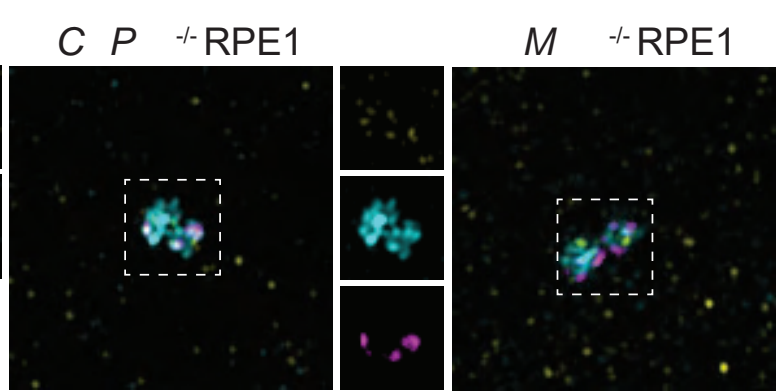

C $P$ - $P^{-R P E} 1$
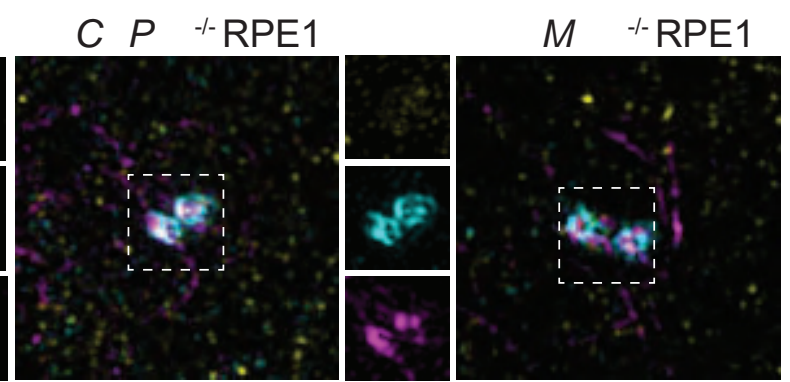

C $P$ - $\quad$ RPE1

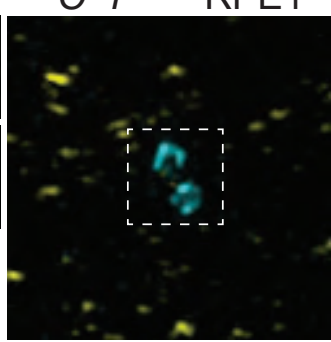

$M \quad$-l-RPE1
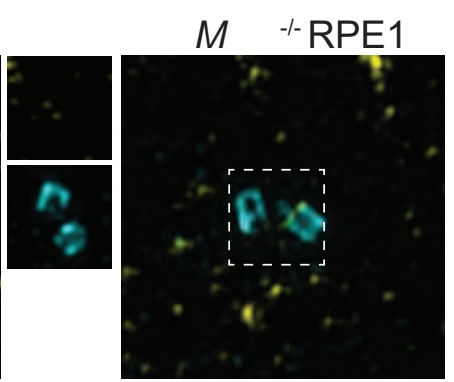

b

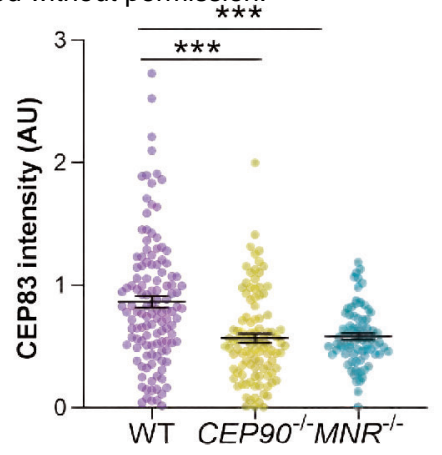

d
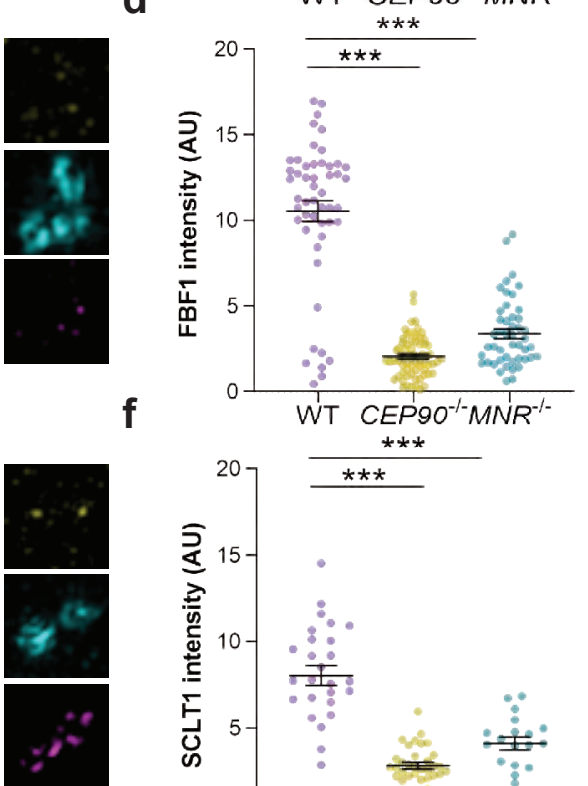

h
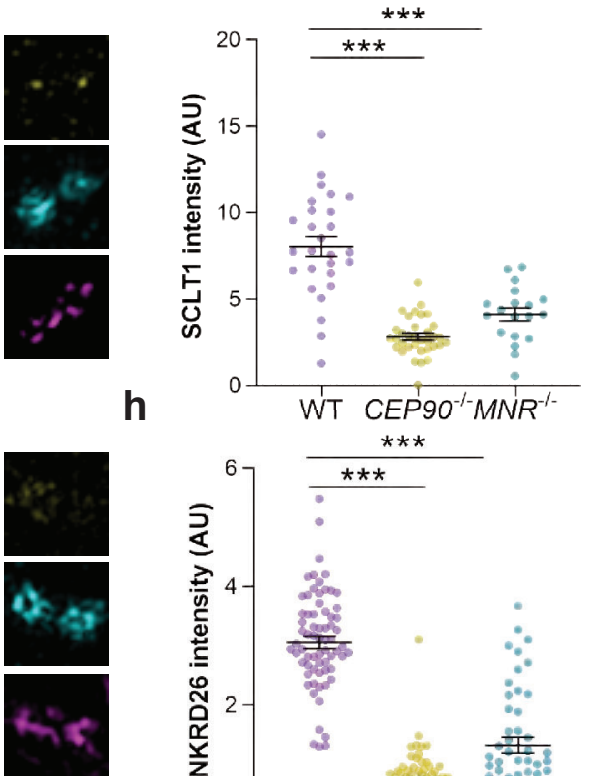

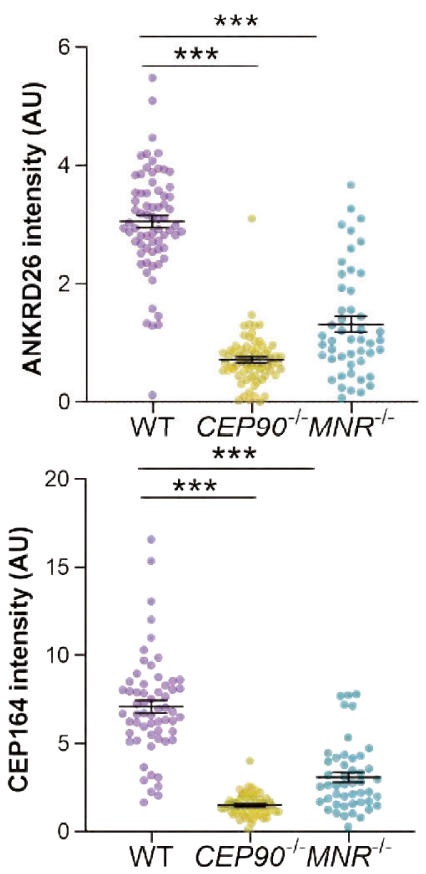




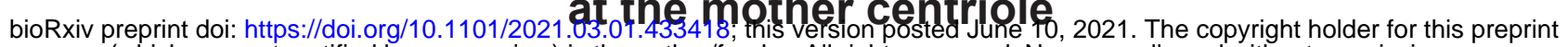
(which was not certified by peer review) is the author/funder. All rights reserved. No reuse allowed without permission.

a WT RPE1
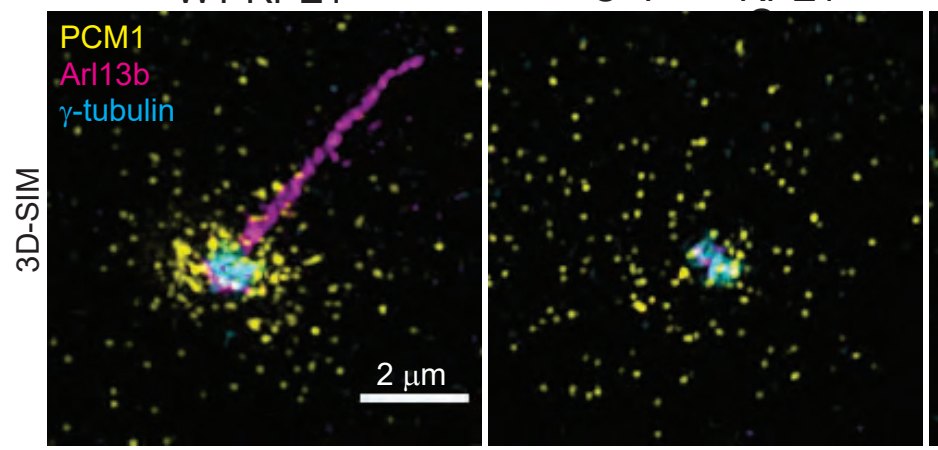

d

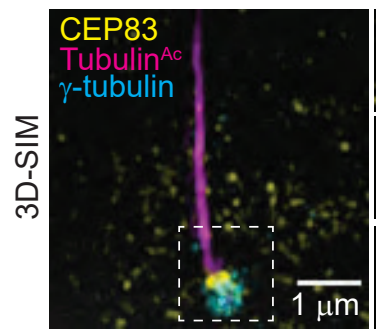

f WT RPE1

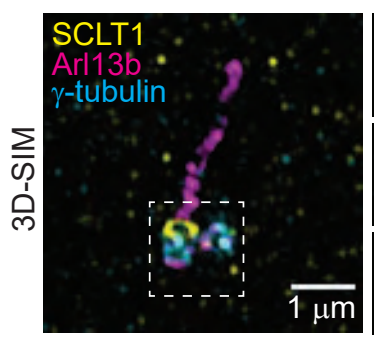

h WT RPE1

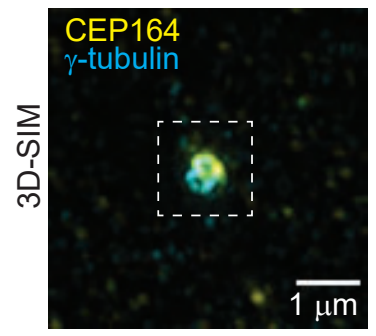

PCM1-1-RPE1
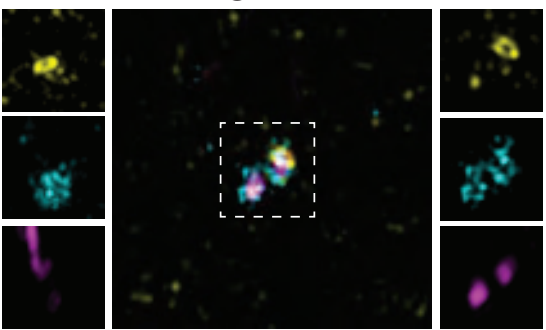

PCM1-1-RPE1
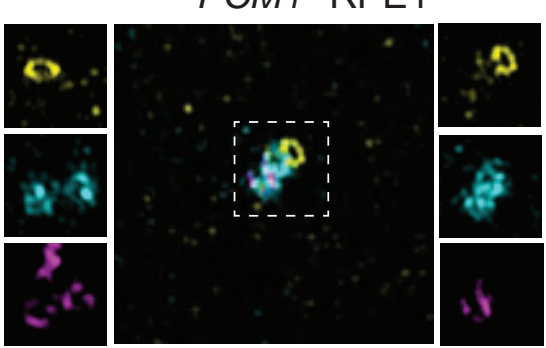

PCM1--RPE1
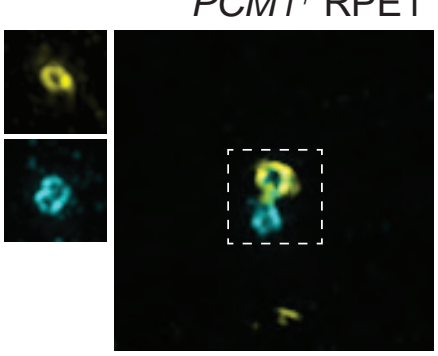

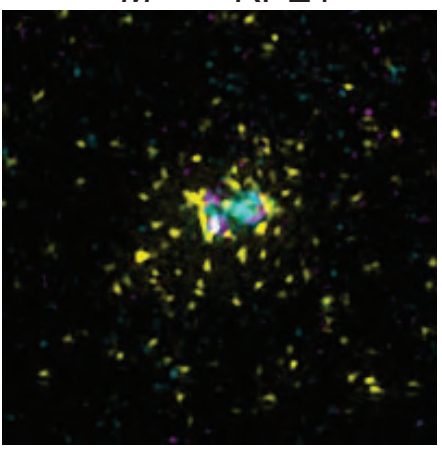

e

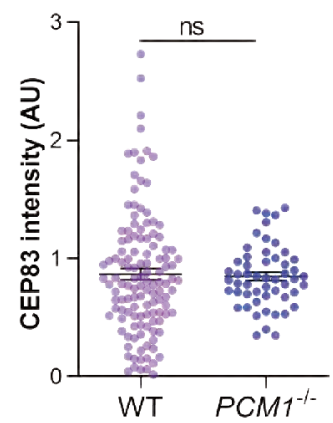

g
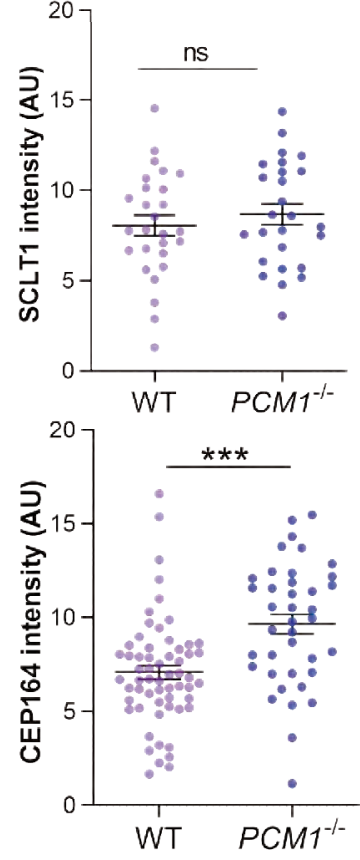
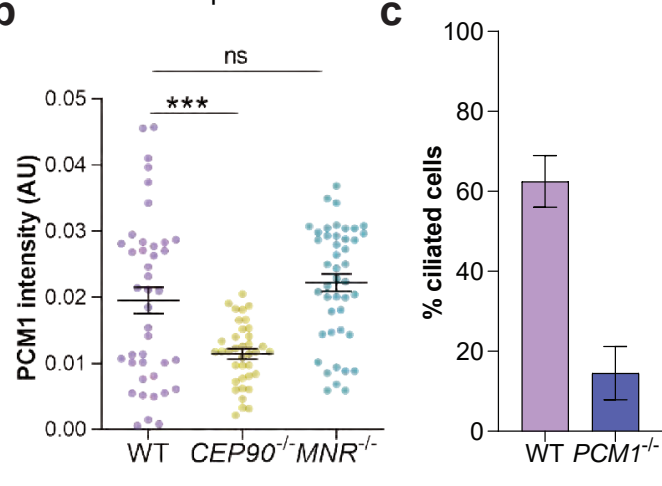

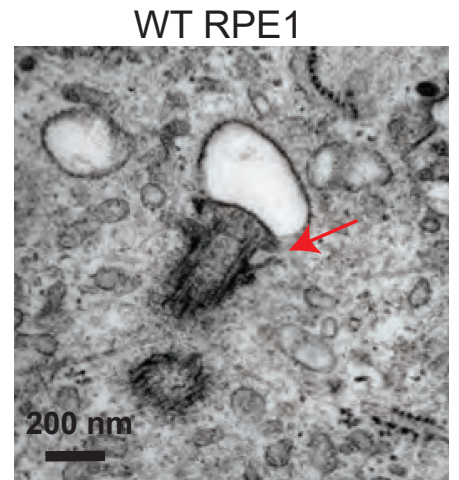

PCM1-- RPE1

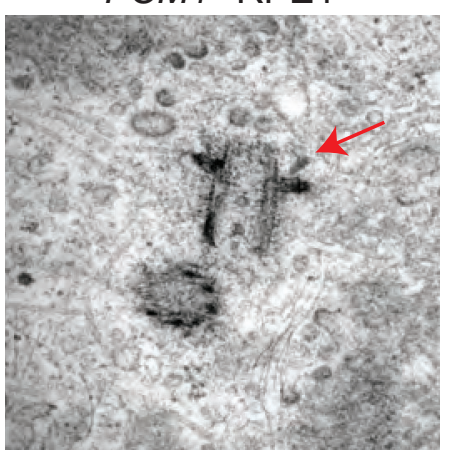




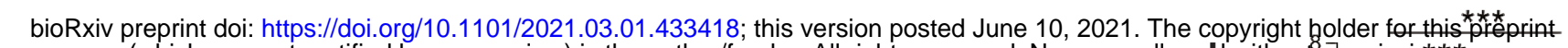

a WT (which was not certified by peer review) is the author/funder. All rights reserved No, reuse allowelo without permissiont*
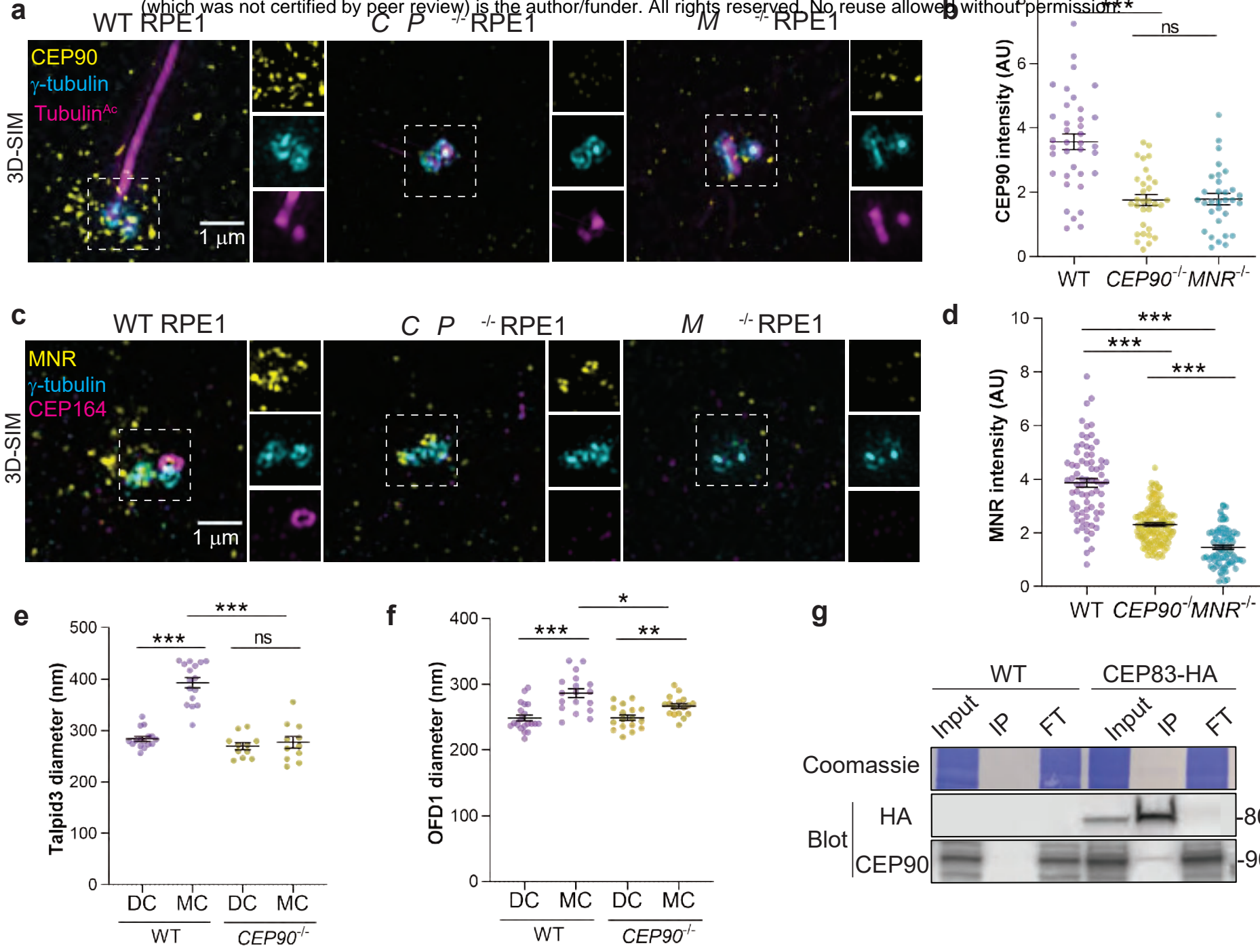

9

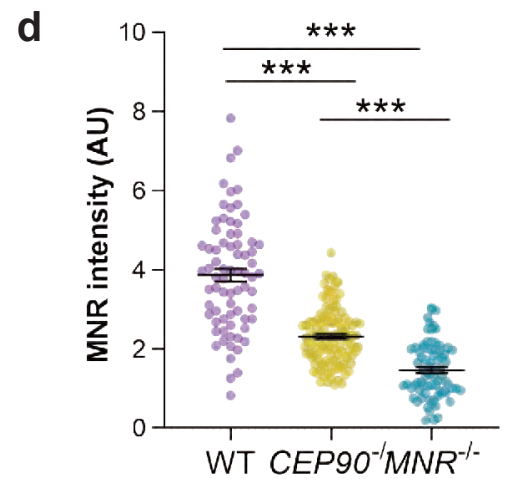

h

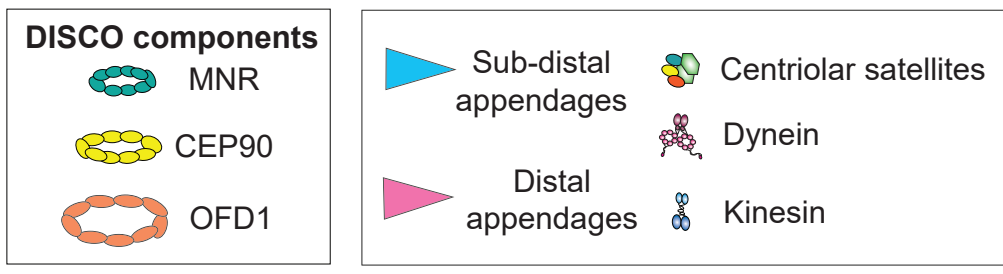

Recruitment of OFD1 Recruitment of CEP90 Recruitment of CEP83 by MNR by MNR by CEP90

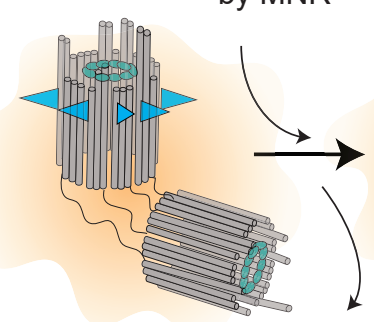

Regulation of centriole length
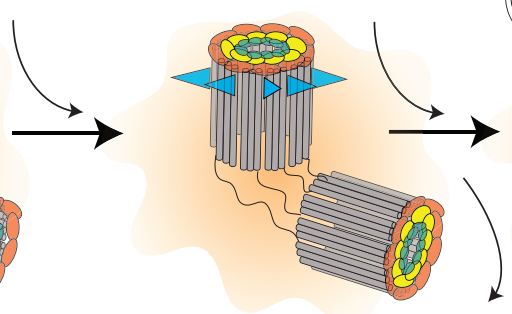

Formation of distal appendages

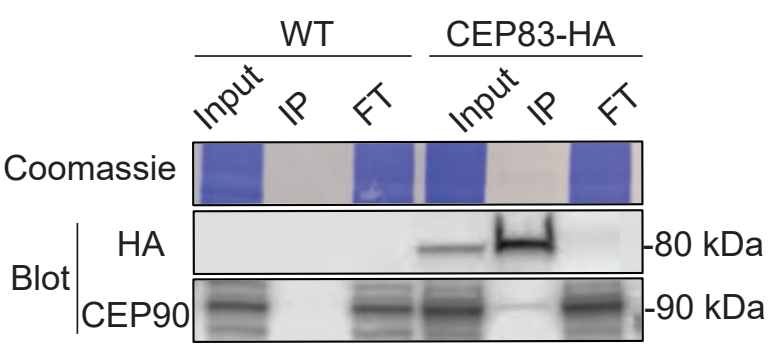


a $\quad C \quad P^{+/+}$allele: 5'- TAGTTTAGAAACAACAGTTCCTACGGATGATATTTCCTCATCAGAAGAG -3'

C $P^{+/+}$translate : MSRKISKESKKVNISSSLESEDISLETTVPTDDISSSEEREGKVRI----

$C P$-- allele 1: 5'- TAGTTTAGAAACAACAGTTCCTA--|GATGATATTTCCTCATCAGAAGAG -3'

C $P \quad$-- allele 1 translate: MSRKISKESKKVNISSSLESEDISLETTVPR*

C $P \quad$-r- allele 2: 5'- TAGTTTAGAAACAACAGTTCCTACG|--TGATATTTCCTCATCAGAAGAG -3'

C $P \quad$-r allele 2 translate: MSRKISKESKKVNISSSLESEDISLETTVPT*

b $M^{+/+}$allele: 5'- TTTGATTCCTTCCTTTCCACCCTGTGTCGGGTATTTTATACTTAGTATGTC -3'

$M^{+/+}$allele translate: MGPGQ---SSQKCGHTKYKIPDHRVERKESKSQAACQCSHQPSKVEISSSGAKVY----

M -- allele 1: 5'- TTTGATTCCTTCCTTTCCACCCTGT|-GTCGGGTATTTTATACTTAGTATGTC -3'

$M \quad$-- allele 1 translate: MGPGQ---SSQKCGHTKYKIPDTGWKGRNQRVKQPVSVATSHPK*

M -- allele 2: 5'- TTTGATTCCTTCCTTTCCACCCTGT|NGGTCGGGTATTTTATACTTAGTATGTC -3'

$M \quad$-- allele 2 translate:MGPGQ---SSQKCGHTKYKIPDXQGGKEGIKESSSLSV*

c $\quad \mathrm{PCM1}^{+/+}$allele : 5'- GATCATCAGAAAAGAATAAGAAAAAGTTTGGTGTAGAAAGTGATAAAAGAG -3'

$P C M 1^{+/+}$allele translate: MATGGGPFEDGMNDQDLPNWSNENVDDRLNNMDWGAQQKKANRSSEKNKKKFGVES---

PCM1/-r- allele : 5'- GATCATCAGAAAAGAATAAGAAAAA|NGTTTGGTGTAGAAAGTGATAAAAGAG -3'

$P C M 1^{-/-}$allele translate: MATGGGPFEDGMNDQDLPNWSNENVDDRLNNMDWGAQQKKANRSSEKNKKXVWCRK*

PAM site

Cut site

- = deletion

$\mathrm{N}=$ insertion

* = stop codon

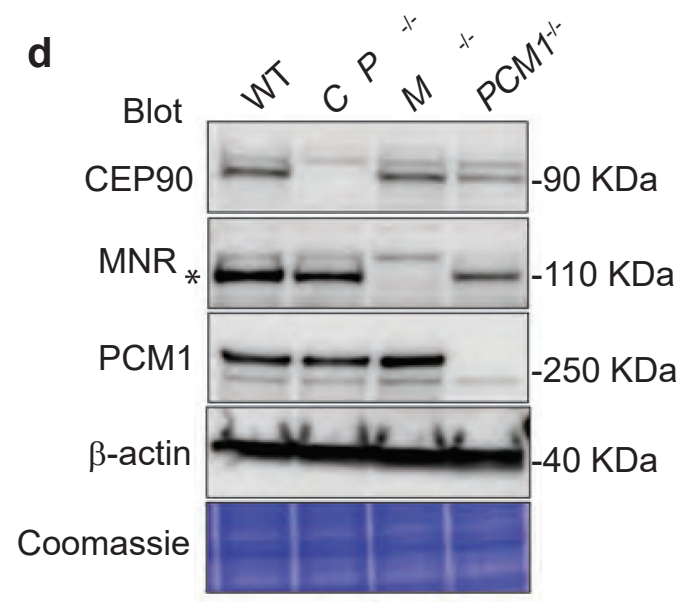




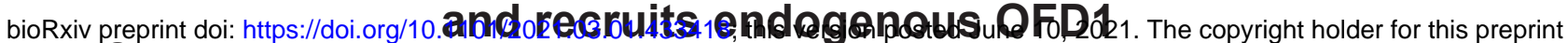
Aunich was not certified by peer review) is the author/funder. All riahts reserved. No reuse allowed without permission.
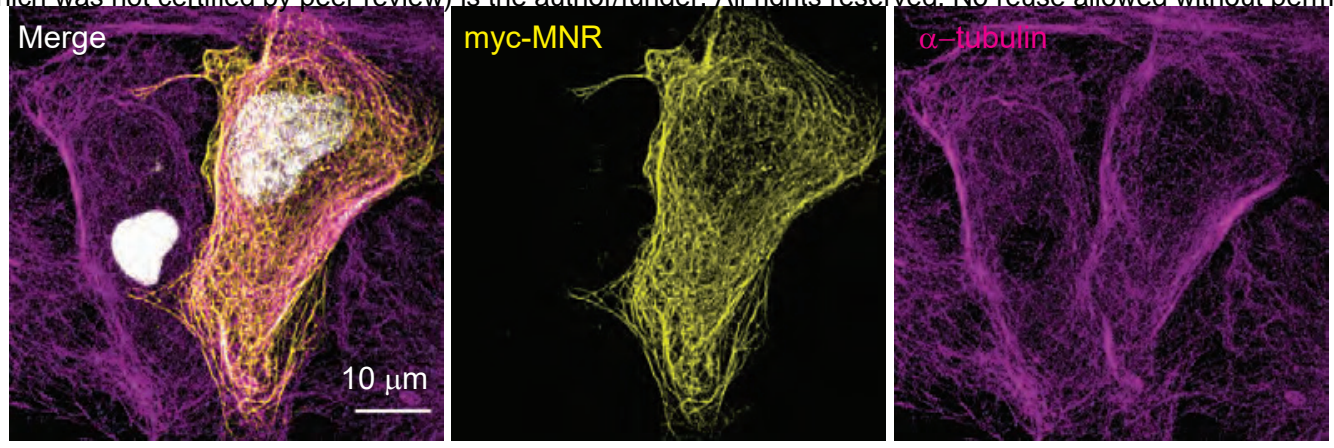

b
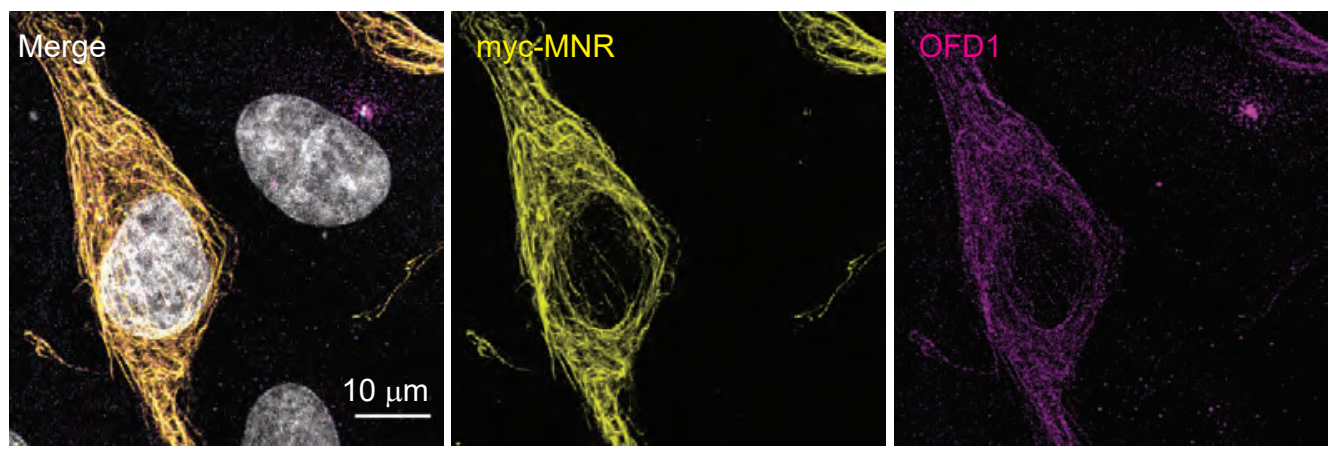

C
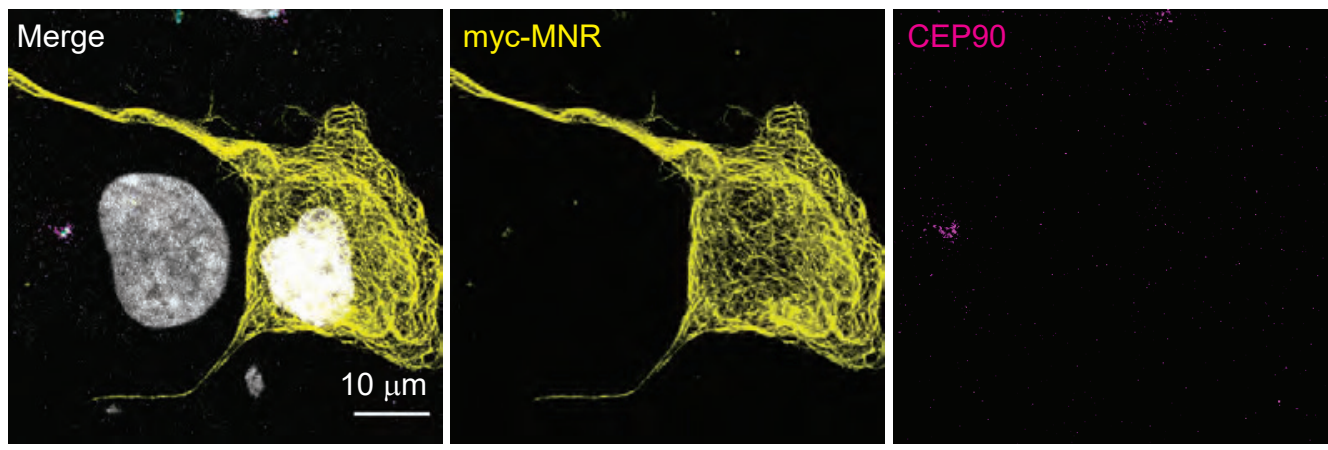

d
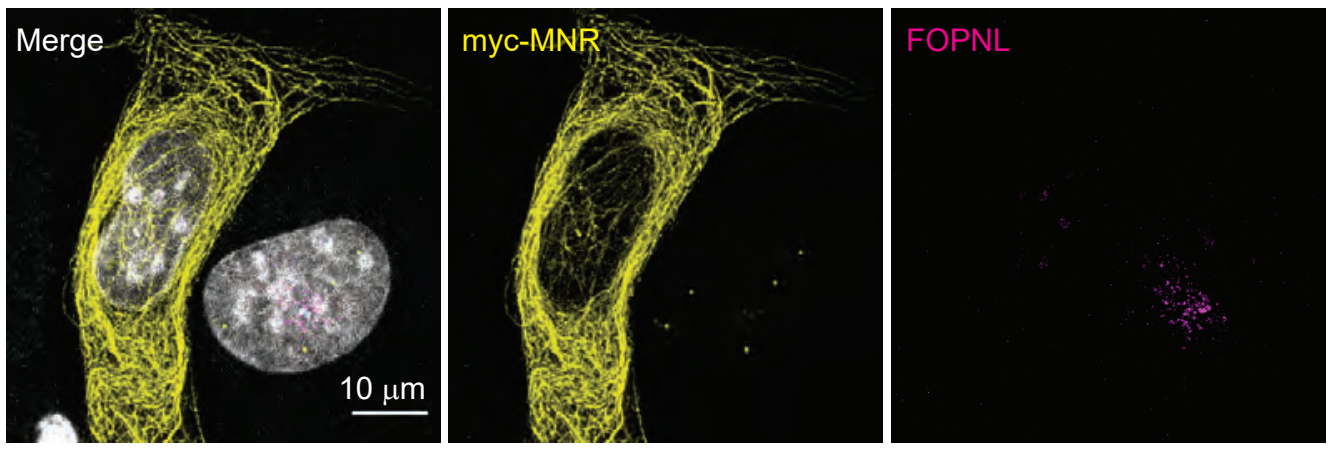

a WT RP twhich was not certified by per reveris the author/funder. Al rights

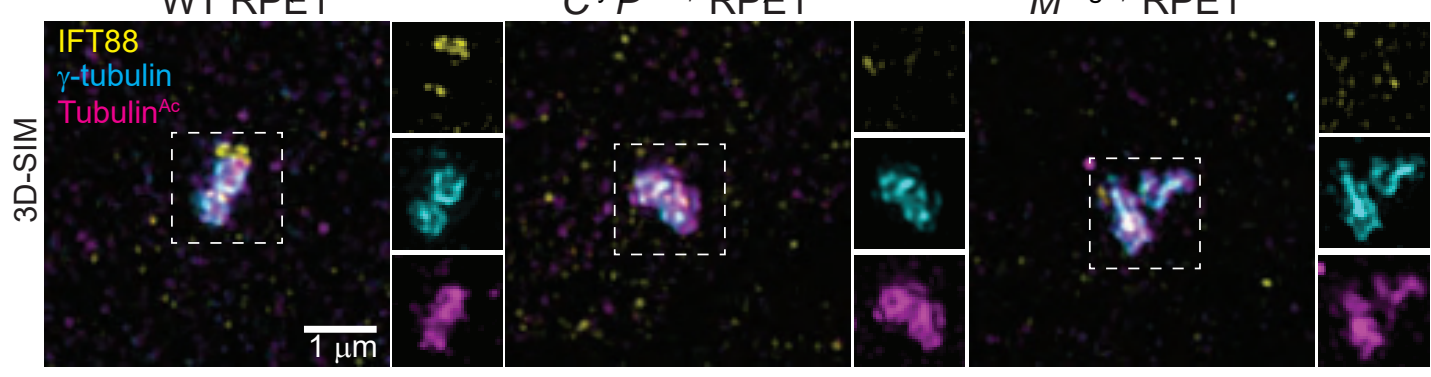

C

c WT RPE1
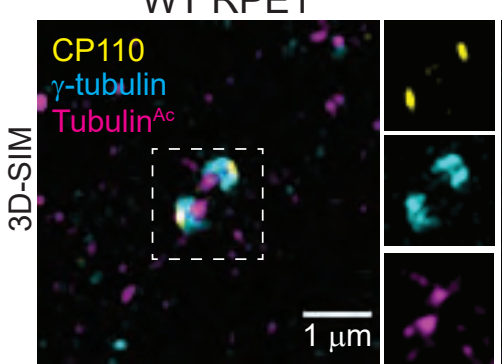

C $P$ P $\quad$-l-RPE1
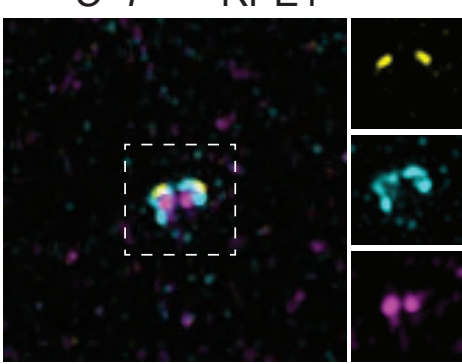

$M \quad{ }^{-/}$RPE1

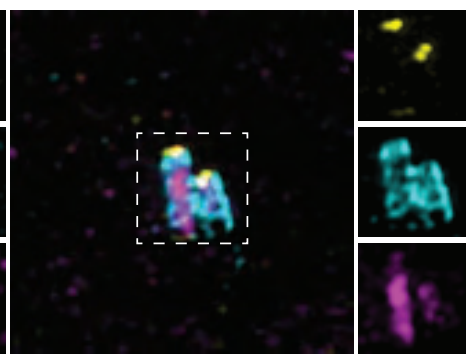

e
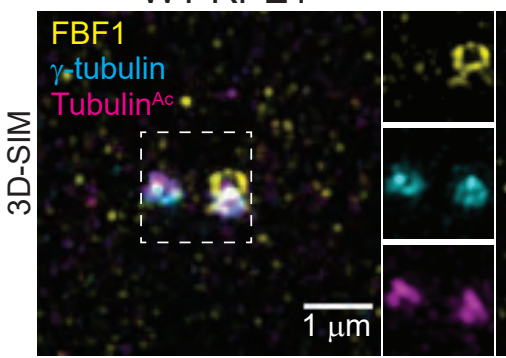

C $P$ - $\quad$ RPE1

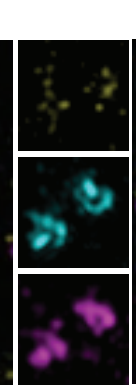

g

क

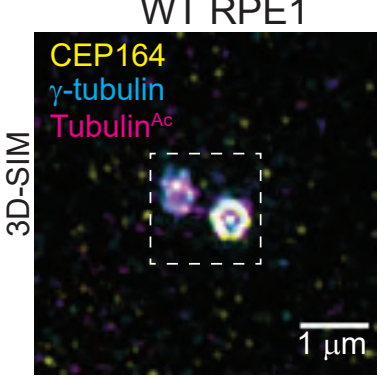

C $P$ - $\quad$ - RPE1

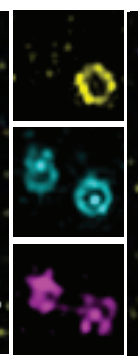

$M \quad$ - RPE1

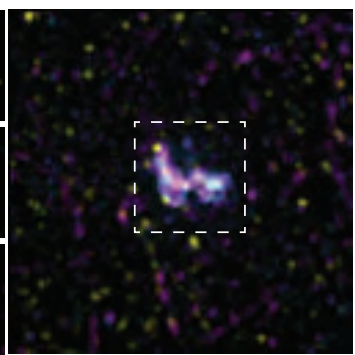

$M \quad$ - RPE1

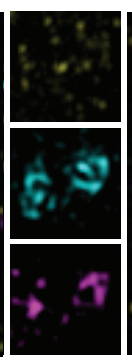

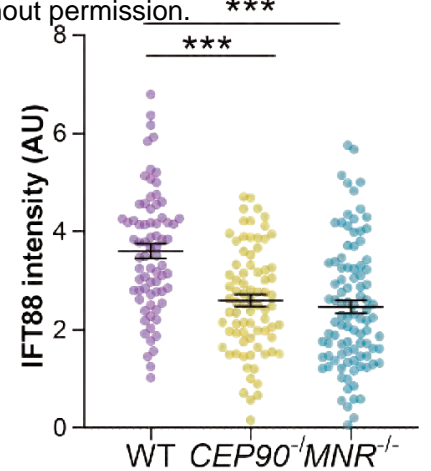

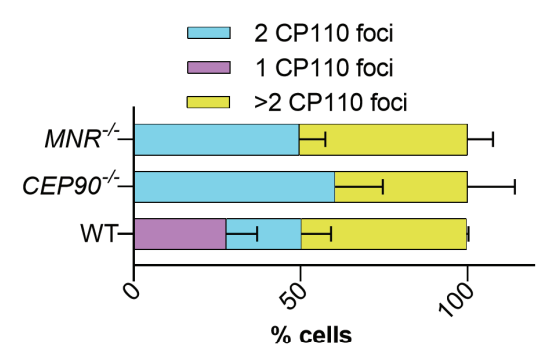

f

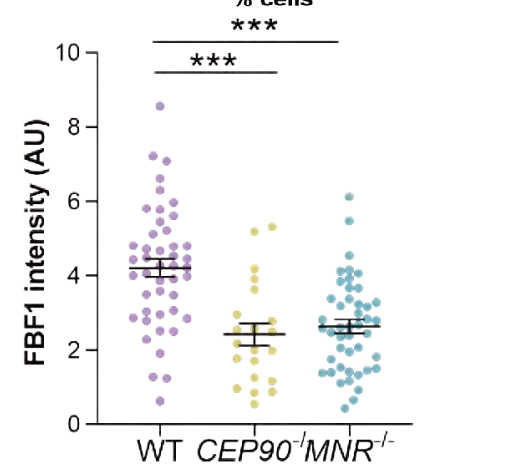

h

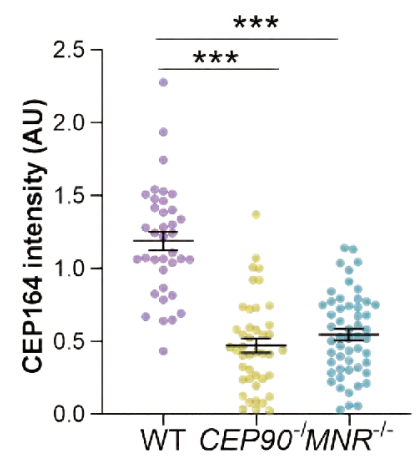


bioRxiv preprint doi: https://doi.org/10.1101/2021.03.01.433418; this version posted June 10,2021 . The copyright holder for this preprint

(whicherdadtsentidabispeer review) is the author/funder. All rights reserved. No reuse allowed without permission.

a
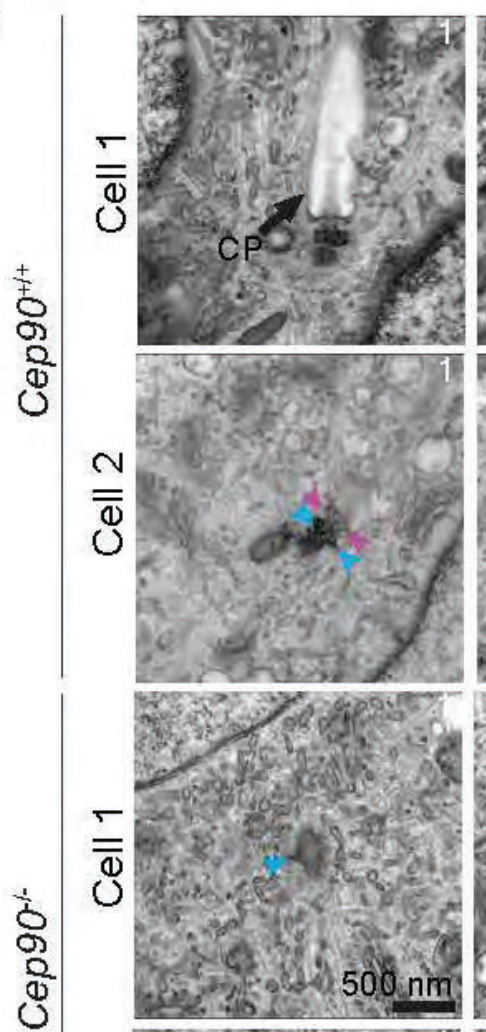

b
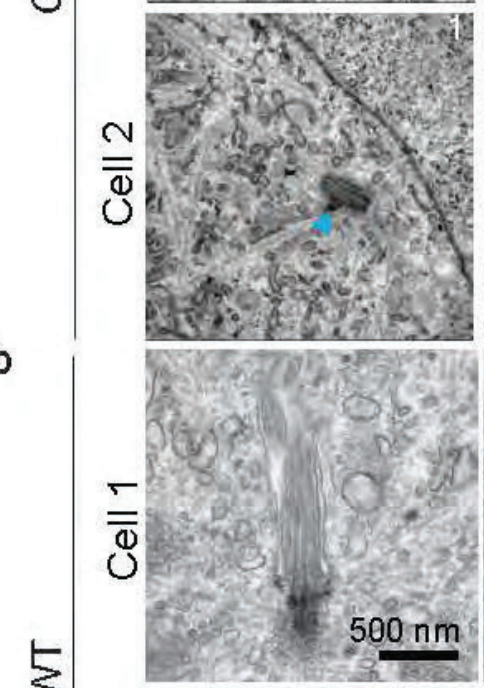

$\leq$
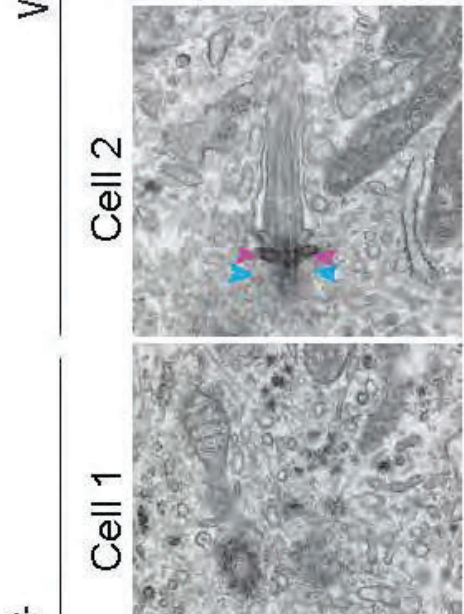
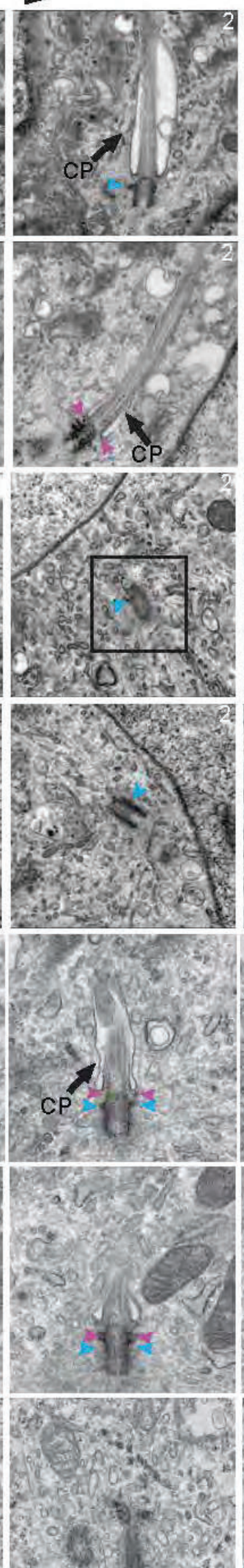

$\sum_{j}^{\frac{1}{d}}$
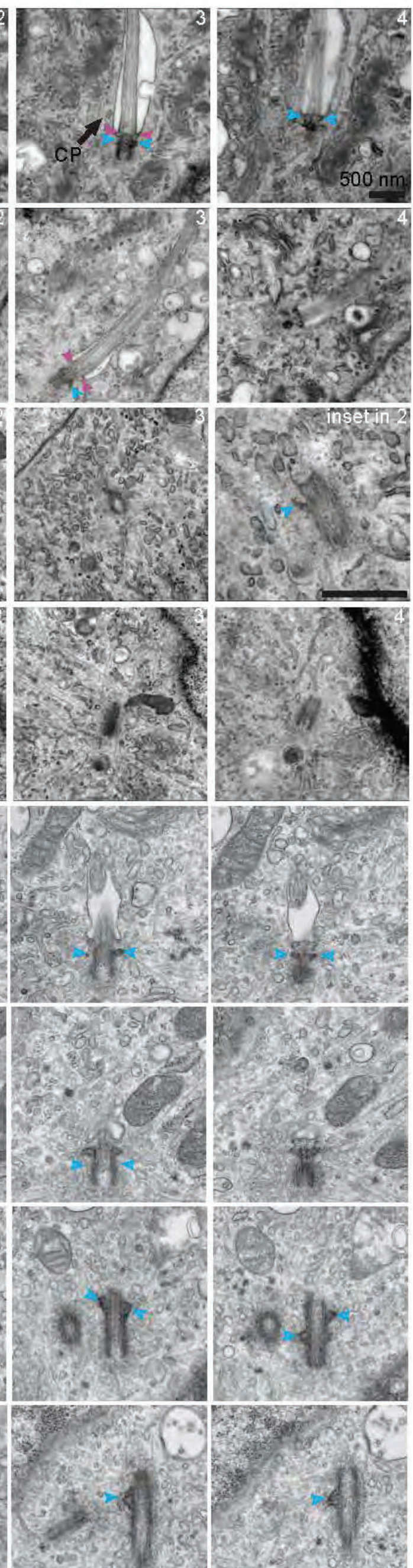


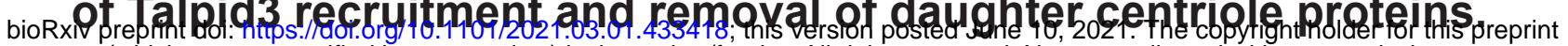

(which was not certified by peer review) is the author/funder. All rights reserved. No reuse allowed without permission.

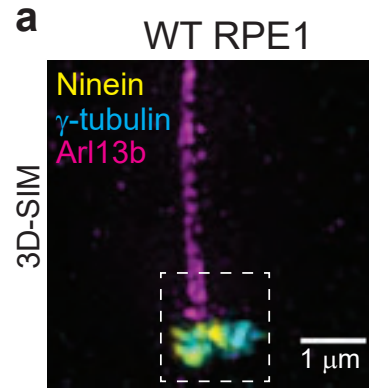

\section{WT RPE1}
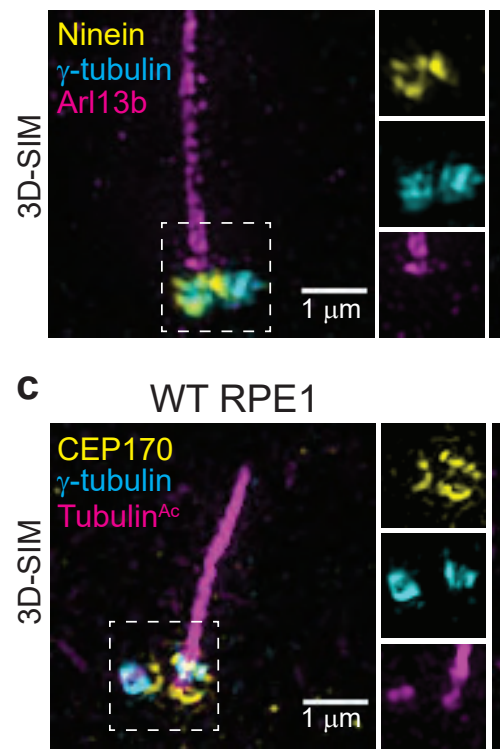

C $P$-/RPE1

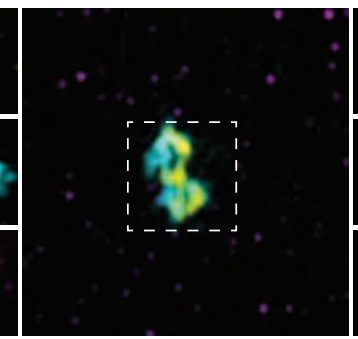

C $P^{-/-R P E 1}$

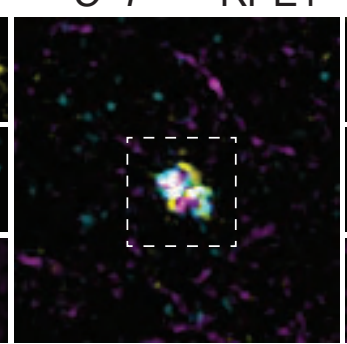

e

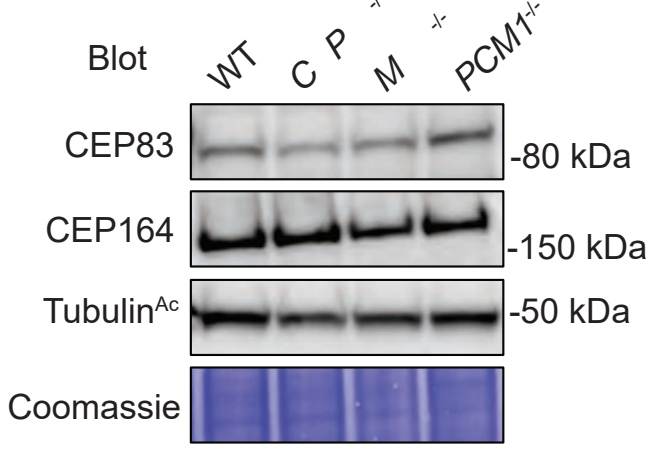

g

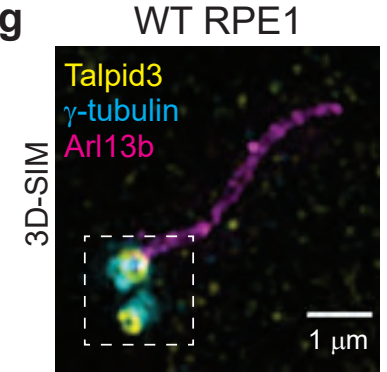

i

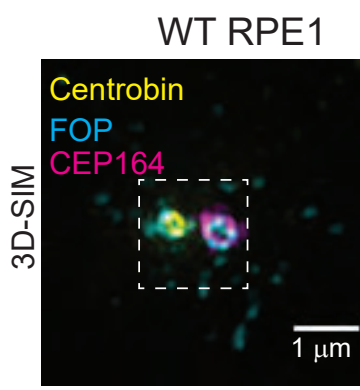

k

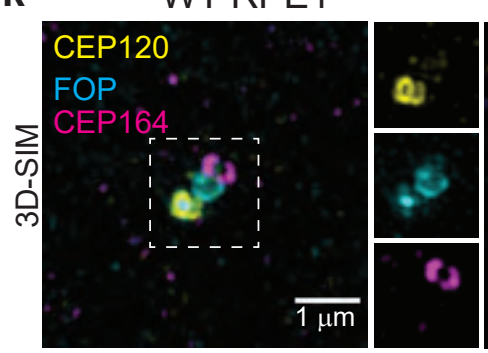

C $P$ - RPE1
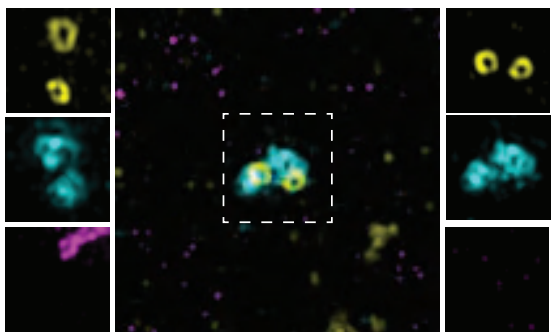

C $P$ - $\quad$ RPE1

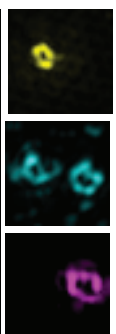

C $P^{-/-R P E 1}$
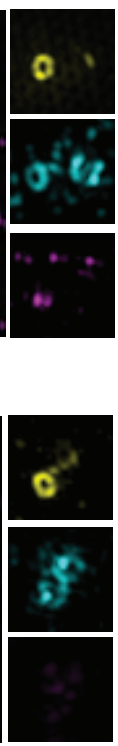

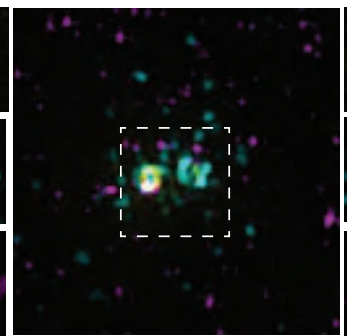

$M \quad$-/RPE1
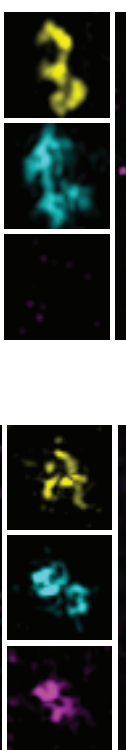

M

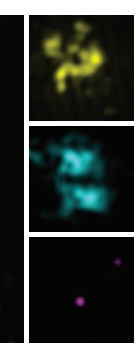

d

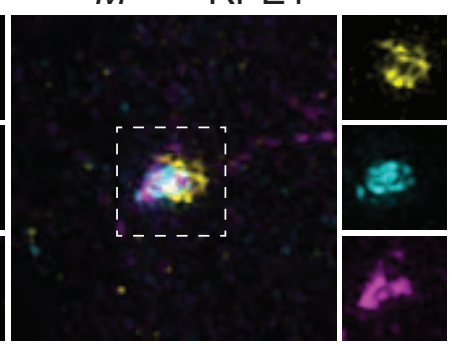

b

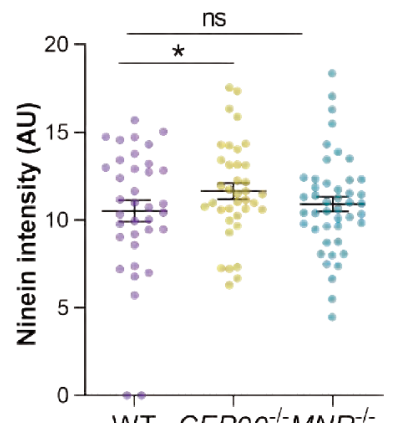

WT CEPGO-i- $\mathrm{MNR}^{-1-}$

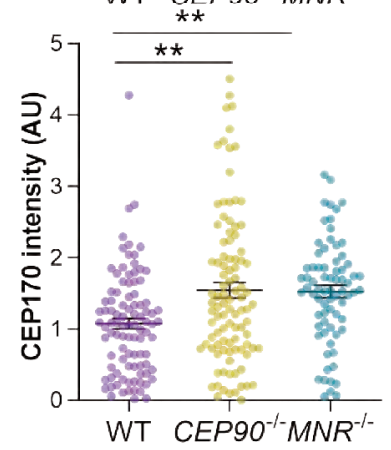

f

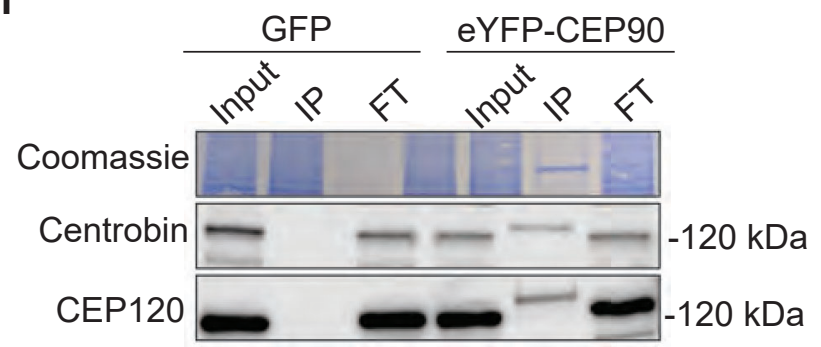

$M \quad$ - RPE1

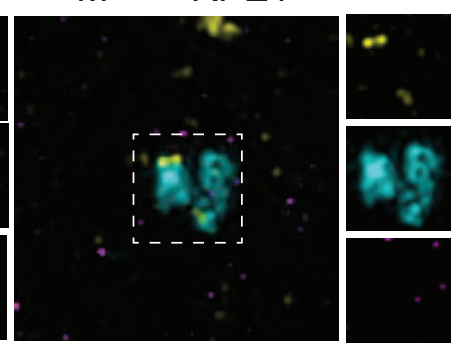

$M \quad$ - RPE1

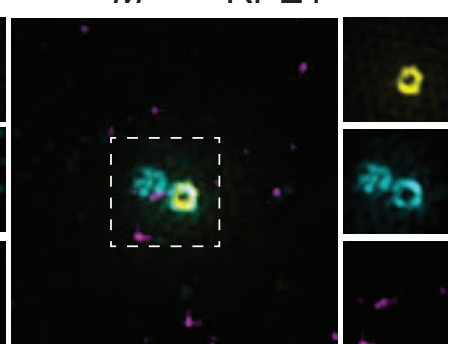

$M \quad$-l-RPE1 h

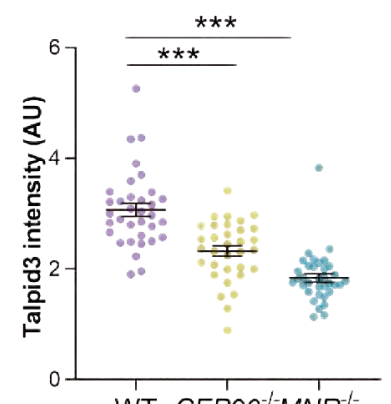

j

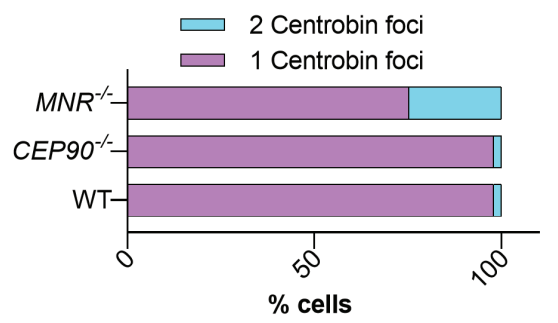

I

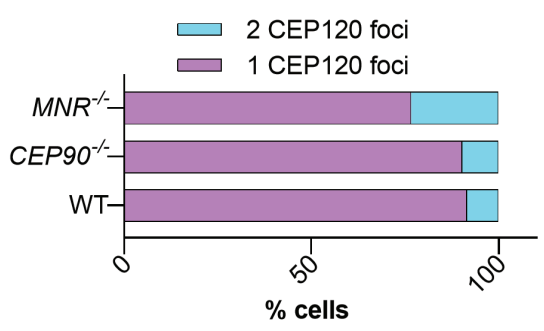


Table 1. List of primary antibodies used in this study for immunofluorescence (IF) and western blotting (WB)

\begin{tabular}{|c|c|c|c|c|c|}
\hline Antibody & Host & Source & $\begin{array}{l}\text { Catalog } \\
\text { Number }\end{array}$ & $\begin{array}{c}\text { Dilution/Fixation } \\
\text { (IF) }\end{array}$ & $\begin{array}{l}\text { Dilution } \\
\text { (WB) }\end{array}$ \\
\hline CEP90 & Rabbit & Proteintech & 14413-1-AP & & $1: 500$ \\
\hline CEP90 & Rabbit & Kunsoo Rhee ${ }^{1}$ & & 1:500/MeOH & \\
\hline MNR & Rat & Olivier Rosnet ${ }^{2}$ & & 1:500/MeOH & \\
\hline MNR & Rabbit & Novus Biologicals & NBP1-90929 & & $1: 500$ \\
\hline OFD1 & Rabbit & Novus Biologicals & NBP1-89355 & 1:500/MeOH & $1: 500$ \\
\hline FOPNL & Rabbit & Sigma Prestige & HPA040599 & 1:100/MeOH & \\
\hline CEP164 & Goat & $\begin{array}{l}\text { Santa Cruz } \\
\text { Biotechnology }\end{array}$ & sc-240226 & 1:100/MeOH & \\
\hline CEP164 & Mouse & Novus Biologicals & NBP2-43707 & & $1: 2000$ \\
\hline y-tubulin & Mouse & Sigma GTU-88 & T6557 & 1:500/MeOH & \\
\hline Tubulin ${ }^{A c}$ & Rabbit & $\begin{array}{l}\text { Cell Signaling } \\
\text { Technology }\end{array}$ & 5335T & 1:2000/MeOH & \\
\hline Tubulin ${ }^{A c}$ & Mouse & $\begin{array}{l}\text { Santa Cruz } \\
\text { Biotechnology }\end{array}$ & $\begin{array}{l}\text { sc-23950 } \\
\text { AF647 }\end{array}$ & 1:100/MeOH & \\
\hline a-tubulin & Mouse & Sigma Aldrich & T5168 & 1:1000/PFA & \\
\hline$\beta$-actin & Mouse & Proteintech & 6008-1-lg & & $1: 100,000$ \\
\hline GAPDH & Mouse & Proteintech & 60004-1-lg & & $1: 100,000$ \\
\hline FBF1 & Rabbit & Proteintech & 11531-1-AP & 1:100/MeOH & \\
\hline ANKRD26 & Rabbit & GeneTex & GTX128255 & 1:100/MeOH & \\
\hline CEP83 & Rabbit & Sigma Prestige & HPA038161 & $1: 100 / \mathrm{MeOH}$ & \\
\hline CENPF & Mouse & BD Biosciences & 610768 & 1:100/MeOH & \\
\hline Myo-Va & Rabbit & Novus Biologicals & NBP1-92156 & $1: 100 / \mathrm{MeOH}$ & \\
\hline CP110 & Mouse & Millipore & MABT1354 & 1:100/MeOH & \\
\hline CEP97 & Rabbit & Proteintech & 22050-1-AP & $1: 100 / \mathrm{MeOH}$ & \\
\hline IFT88 & Rabbit & Proteintech & 13967-1-AP & 1:100/MeOH & \\
\hline FOP & Rabbit & Proteintech & 11343-1-AP & 1:500/MeOH/ PFA & \\
\hline PCM1 & Rabbit & Proteintech & 19856-1-AP & & $1: 500$ \\
\hline PCM1 & Mouse & Santacruz & sc-398365 & 1:100/MeOH & \\
\hline Talpid3 & Rabbit & Proteintech & 24421-1-AP & 1:100/MeOH & \\
\hline Centrobin & Mouse & Abcam & Ab70448 & 1:100/MeOH & $1: 1000$ \\
\hline Ninein & Rabbit & Michel Bornens & L79 & $1: 200 / \mathrm{MeOH}$ & \\
\hline ODF2 & Mouse & Novus Biologicals & $\begin{array}{l}\text { H00004957- } \\
\text { M01 }\end{array}$ & 1:100/MeOH & \\
\hline CEP170 & Mouse & ThermoFisher & $72-413-1$ & 1:100/ MeOH & \\
\hline Myc-tag & Goat & Novus Biologicals & NB600-335 & $1: 500 /$ PFA & \\
\hline Arl13b & Rabbit & Proteintech & 177100-1-AP & 1:500/MeOH/ PFA & \\
\hline mNeonGreen & Mouse & Chromotek & $32 \mathrm{~F} 6$ & 1:500/PFA & \\
\hline Talpid3 & Rabbit & Proteintech & 24421-1-AP & $1: 100 / \mathrm{MeOH}$ & \\
\hline CEP162 & Rabbit & Sigma Prestige & HPA030170 & 1:100/MeOH & \\
\hline CEP131 & $\begin{array}{l}\text { Guinea } \\
\text { pig }\end{array}$ & Kodani et al.,2015 3 & & 1:100/PFA/ MeOH & \\
\hline CEP120 & Rabbit & Betleja et al., $2018^{4}$ & & 1:3000/MeOH & \\
\hline
\end{tabular}




\section{REFERENCES}

1. Kim, K. \& Rhee, K. The pericentriolar satellite protein CEP90 is crucial for integrity of the mitotic spindle pole. Cell Sci 124, 338-347 (2011).

2. Chevrier, V. et al OFIP/KIAA0753 forms a complex with OFD1 and FOR20 at pericentriolar satellites and centrosomes and is mutated in one individual with oral-facial-digital syndrome. Hum Mol enet 25, 497-513 (2016).

3. Kodani, A. et al Centriolar satellites assemble centrosomal microcephaly proteins to recruit CDK2 and promote centriole duplication. Ii e 4, e07519 (2015).

4. Betleja, E., Nanjundappa, R., Cheng, T. \& Mahjoub, M. R. A novel cep120-dependent mechanism inhibits centriole maturation in quiescent cells. li e 7, (2018). 